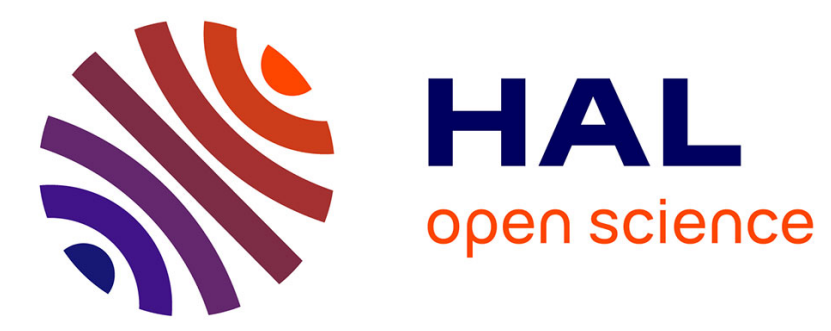

\title{
A 'reciprocal' theorem for the prediction of loads on a body moving in an inhomogeneous flow at arbitrary Reynolds number
}

Jacques Magnaudet

\section{- To cite this version:}

Jacques Magnaudet. A 'reciprocal' theorem for the prediction of loads on a body moving in an inhomogeneous flow at arbitrary Reynolds number. Journal of Fluid Mechanics, 2011, vol. 689, pp.564-604. 10.1017/jfm.2011.363 . hal-00907975

\section{HAL Id: hal-00907975 \\ https://hal.science/hal-00907975}

Submitted on 22 Nov 2013

HAL is a multi-disciplinary open access archive for the deposit and dissemination of scientific research documents, whether they are published or not. The documents may come from teaching and research institutions in France or abroad, or from public or private research centers.
L'archive ouverte pluridisciplinaire HAL, est destinée au dépôt et à la diffusion de documents scientifiques de niveau recherche, publiés ou non, émanant des établissements d'enseignement et de recherche français ou étrangers, des laboratoires publics ou privés. 


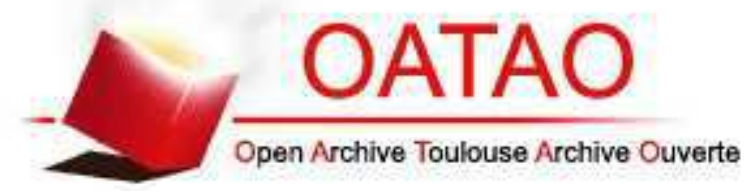

This is an author-deposited version published in : http://oatao.univ-toulouse.fr/ Eprints ID : 10259

To link to this article : DOI:10.1017/jfm.2011.363

URL : http://dx.doi.org/10.1017/jfm.2011.363

To cite this version :

Magnaudet, Jacques A 'reciprocal' theorem for the prediction of loads on a body moving in an inhomogeneous flow at arbitrary Reynolds number. (2011) Journal of Fluid Mechanics, vol. 689 . pp. 564-604. ISSN 0022-1120

Any correspondence concerning this service should be sent to the repository administrator: staff-oatao@ listes.diff.inp-toulouse.fr 


\title{
A 'reciprocal' theorem for the prediction of loads on a body moving in an inhomogeneous flow at arbitrary Reynolds number
}

\author{
Jacques Magnaudet $\dagger$ \\ Institut de Mécanique des Fluides de Toulouse, UMR CNRS/INPT/UPS 5502, Allée Camille Soula, \\ 31400 Toulouse, France
}

\begin{abstract}
Several forms of a theorem providing general expressions for the force and torque acting on a rigid body of arbitrary shape moving in an inhomogeneous incompressible flow at arbitrary Reynolds number are derived. Inhomogeneity arises because of the presence of a wall that partially or entirely bounds the fluid domain and/or a non-uniform carrying flow. This theorem, which stems directly from Navier-Stokes equations and parallels the well-known Lorentz reciprocal theorem extensively employed in low-Reynolds-number hydrodynamics, makes use of auxiliary solenoidal irrotational velocity fields and extends results previously derived by Quartapelle \& Napolitano (AIAA J., vol. 21, 1983, pp. 911-913) and Howe (Q. J. Mech. Appl. Maths, vol. 48, 1995, pp. 401-426) in the case of an unbounded flow domain and a fluid at rest at infinity. As the orientation of the auxiliary velocity may be chosen arbitrarily, any component of the force and torque can be evaluated, irrespective of its orientation with respect to the relative velocity between the body and fluid. Three main forms of the theorem are successively derived. The first of these, given in (2.19), is suitable for a body moving in a fluid at rest in the presence of a wall. The most general form (3.6) extends it to the general situation of a body moving in an arbitrary non-uniform flow. Specific attention is then paid to the case of an underlying timedependent linear flow. Specialized forms of the theorem are provided in this situation for simplified body shapes and flow conditions, in (3.14) and (3.15), making explicit the various couplings between the body's translation and rotation and the strain rate and vorticity of the carrying flow. The physical meaning of the various contributions to the force and torque and the way in which the present predictions reduce to those provided by available approaches, especially in the inviscid limit, are discussed. Some applications to high-Reynolds-number bubble dynamics, which provide several apparently new predictions, are also presented.
\end{abstract}

Key words: general fluid dynamics, vortex dynamics

\section{Introduction}

Given its many applications in all sorts of domains (e.g. oceanography, meteorology, flight dynamics, and mechanical, chemical and marine engineering, to mention 
just a few), predicting the forces and torques acting on bodies moving in an arbitrary flow field has always been a central concern in fluid mechanics. General formulations of the problem have been developed in the two limits where the governing equations are linear, namely Stokes flows and potential flows. The general case where inertial and viscous effects are both present poses much greater difficulties, owing to the nonlinear interplay between the various contributions involved. In particular, the determination of the pressure distribution at the body surface is generally inordinately difficult, and most attempts try to by-pass it by expressing the hydrodynamic loads on the body as the sum of various surface and volume integrals involving solely the velocity field and its derivatives, especially the local vorticity.

Among these approaches, the so-called 'dissipation' theorem has been found to be useful for evaluating the viscous drag acting on bubbles rising in a liquid at rest, especially in the high-Reynolds-number regime (Levich $\square, \square$; Batchelor ); some extensions to drops have also been considered (Harper \& Moore $\square$ ). Basically, this theorem states that the rate at which the drag force works just balances the viscous dissipation in the entire flow. In this regime, the boundary layer induced by the shear-free condition at the bubble surface only induces secondary changes in the flow field, in contrast to what happens with solid bodies. For that reason, at leading order the flow can be considered irrotational everywhere except right at the bubble surface. It is then a simple matter to calculate the dissipation throughout the flow and deduce the drag. The calculation of this force through a direct integration of the surface stress was performed by Kang \& Leal ( $\square$ ) for a spherical bubble and offers a good view of the technical difficulties related to the evaluation of viscous corrections in the pressure field.

The major limitation of the 'dissipation' theorem is that it only gives access to the drag force. Indeed, since the lift component of the force does not produce any work, it does not enter the kinetic energy budget of the flow. The same problem arises for the torque components in directions about which the body does not rotate. For these reasons, a more general theorem from which any component of the force and torque could be obtained, irrespective of its orientation with respect to the relative motion of the body in the fluid, appears desirable. In the Stokes flow regime, this goal is classically achieved with the Lorentz reciprocal theorem (Lorentz $\square$ ). In its application to the determination of forces and torques on moving bodies, this theorem makes crucial use of auxiliary velocity and stress fields, which are easier to evaluate than the primary fields of direct interest. The auxiliary velocity fields, which satisfy both the kinematic and dynamic boundary conditions at the body surface as well as on any other surface that may bound the flow domain, correspond to body motions performed in a fluid at rest with a unit velocity or rotation rate in an arbitrary direction. Lorentz's reciprocal theorem has, for instance, been extensively used to evaluate the Stokes resistance of a particle of arbitrary shape (Brenner $\square$ ), the inertia-induced lateral migration of rigid particles in wall-bounded flows (Cox \& Brenner $\square$; Ho \& Leal $\square$; Vasseur \& Cox $\square$ ) and the impact of surface deformation on the near-wall migration of deformable drops and bubbles (Chan \& Leal $\square$ ) or on the propulsion of microorganisms (Stone \& Samuel $\square$ ).

From a mathematical viewpoint, Lorentz's reciprocal theorem is the counterpart of Green's well-known second identity for harmonic functions: both formulations involve two mathematical solutions satisfying the same governing equation (the Stokes equation for Lorentz's reciprocal theorem, the Laplace equation for Green's identity) and eliminate the product of their gradients by considering the difference between two dot products involving both solutions (Pozrikidis $\square$ ). This remark suggests 
that it might be possible to build a useful finite-Reynolds-number version of the reciprocal theorem by using auxiliary velocity fields derived from harmonic functions. Such auxiliary velocity fields are thus irrotational and can only satisfy the kinematic boundary condition at the body surface. Hence in such an approach the primary and auxiliary velocity fields do not exactly satisfy the same set of boundary conditions (this is why the word 'reciprocal' is put in quotation marks throughout the paper). Nevertheless it is clear that potential flow theory provides the only tractable solutions that can approach the actual structure of high-Reynolds-number flows through most of the fluid domain. It is therefore both mathematically relevant and practically natural to think of irrotational solutions as the auxiliary velocity fields required to duplicate the creeping flow approach based on Lorentz's reciprocal theorem at high (actually arbitrary) Reynolds number.

The approach outlined above is that developed throughout this paper. It turns out that, from a technical viewpoint, this approach has already been explored in the case of a fluid at rest at infinity (or of a uniform carrying flow), although the motivations were somewhat different and the parallel with Lorentz's reciprocal theorem was not invoked. The initial step was performed by Quartapelle \& Napolitano ( $\square)$, who recognized that the contribution of the pressure field on the body surface can be expressed solely in terms of the velocity field by projecting the Navier-Stokes equations onto the subspace of solenoidal irrotational velocity fields. This result enabled them to obtain the force and torque acting on a translating rigid body as the sum of three distinct contributions. The first of these is a surface integral involving the time rate of change of the body velocity. Since this term is the only one involving the time derivative of the velocity, it corresponds to nothing but the familiar added-mass effects. A second surface integral involves the vorticity at the body surface and thus expresses the contribution of viscous effects related to the dynamic boundary condition (often referred to as skin friction effects in the case of a non-slip condition). Finally, a volume integral over the entire fluid domain gathers nonlinear effects.

This stream of ideas was pursued by Howe $(\square)$ and Chang $(\square)$ with the main objective of disentangling the irrotational and vortical contributions to the total force and interpreting these contributions in terms of added-mass, bound and free vorticity effects, respectively. A major step forwards was achieved by Howe $(\square)$, who extended this approach to derive general formulae for the force and torque acting on bodies of arbitrary shape experiencing both translational and rotational motions. This formulation was also extended to the case of multiple bodies by Howe $(\square)$, Grotta Ragazzo \& Tabak ( $\square$ ) and Chang, Yang \& Chu ( $\square$ ). Implementations in numerical simulation approaches, where the auxiliary harmonic functions are part of the computation, owing to the geometrical complexity of the configuration under consideration, have also been carried out (Protas, Styczek \& Nowakowski $\square$; Pan \& Chew $\square$ ).

To put the present work into a broader context, it is worth emphasizing that the aforementioned contributions are part of the general objective of expressing the force and torque on a moving body solely in terms of the velocity and vorticity fields. This quest has been around for almost a century, at least since the pioneering paper of Burgers ( $)$. Since then, important contributions to this subject, where the concepts of body impulse and vortex impulse play a central role, have been provided by $\mathrm{Wu}(\square)$, Lighthill $({ }, \Gamma)$ and Kambe $(\square)$. The various approaches to that problem and their interconnections have recently been comprehensively reviewed by Biesheuvel \& Hagmeijer ( $\square$ ). A broad discussion of the subject, including numerous applications, is provided in $\S 4.5$ of the recent textbook by Howe $(\square)$. 
The main motivation of the present paper is to set up a rational framework for determining the various contributions to the forces and torques acting on bodies moving in inhomogeneous flow fields, the inhomogeneity being due either to velocity gradients in the carrying flow or to the presence of a bounding wall. Part of the objective is to obtain as many contributions as possible in closed form so as to restrict further numerical evaluation of flow-dependent quantities to a minimum and allow for a detailed physical understanding of the couplings between the characteristics of the body (shape, translational and rotational velocities) and those of the carrying flow (e.g. acceleration, strain rate and vorticity). In the moderate- or high-Reynolds-number regime, a complete analytical evaluation of the force and torque on a solid body is generally hopeless, owing to the strength of the boundary layer and wake effects. Inviscid effects may, however, be valuably estimated if a model for the vorticity distribution in the wake is available, such as that provided by the classical lifting line theory for wings of finite span (see also Howe, Lauchle \& Wang ( $\square$ ) for the evaluation of wake-induced fluctuating forces on a sphere). The situation may be different with bubbles of prescribed shape for the aforementioned reasons. In particular, a consequence of the weakness of vortical effects induced by the shearfree condition is that the flow does not separate at high enough Reynolds number (although it may separate and give rise to an unsteady wake in an intermediate range of Reynolds number (see e.g. Magnaudet \& Mougin $\square$ )). For this reason, no-slip and shear-free boundary conditions are both considered during the derivations carried out below. A secondary, more technical point examined during the derivation process regards the conditions under which the contributions provided by the fictitious surface externally bounding the fluid domain become negligibly small as this surface recedes to infinity. Depending on these conditions, which are related to the compactness of the vorticity distribution in the flow, two different final forms of the theorem are obtained. This point may have some bearing on the interpretation of computational predictions of the force and torque obtained through direct numerical simulations, which necessarily make use of a finite control volume.

The structure of the paper is as follows. Section 2 presents the derivation of the theorem in the case where the fluid is at rest far from the body and the flow domain is partially or entirely bounded by a wall. The physical content of the various contributions is discussed as well as the conditions of validity of the two final expressions that are obtained. In $\S 3$ we extend this theorem to the case of a body moving in an arbitrary non-uniform flow. Once this more general form is obtained and its conditions of validity are specified, we focus on the particular case of broad practical interest in which the carrying flow is linear and the size of the body is much smaller than the inhomogeneity length scale of this flow. General expressions for the force and torque acting on bodies of arbitrary shape are established in this situation and compared with available predictions derived in the inviscid limit. These expressions are then specialized to bodies with three perpendicular symmetry planes for which the reduced number of couplings between the effects of translation and rotation enables a simpler physical interpretation of the various inertial contributions.

Section 4 presents some applications of the forms of the theorem derived in $\S \S 2$ and 3 to bubble dynamics in the high-Reynolds-number regime. These applications aim to illustrate the versatility of the corresponding expressions and their ability to predict both the viscous and inertial contributions to the force and torque whatever their orientation with respect to the relative motion of the body and fluid. Some apparently new results are derived in this section, such as the expression of the viscous torque on a bubble rotating in a fluid at rest or the inertial lift force on a cylindrical bubble 
moving parallel to a wall. Final remarks are provided in $\S 5$. The main text is followed by six technical appendices, the first of which examines the kinematic connections between velocity, strain and vorticity on a surface of arbitrary geometry. The other five detail the mathematical steps required to obtain the various forms of the theorems derived in $\S \S 2$ and 3 ; in the course of appendices $\mathrm{C}, \mathrm{D}$ and $\mathrm{E}$, the conditions that the flow must satisfy at large distance of the body for some of these steps to be valid are discussed.

\section{The 'reciprocal' theorem for a body moving in a fluid at rest}

\subsection{Governing equations}

We consider a body of fixed shaped moving with a translational velocity $\boldsymbol{V}$ and a rotation rate $\Omega$ relative to an ambient fluid with uniform density $\rho$ and kinematic viscosity $v$ that fills the volume $\mathscr{V}$ and is at rest at infinity (figure $\Gamma$ ). We normalize distances and velocities by some characteristic length $R$ and velocity $V_{0}$ of the body, respectively, and pressures by $\rho V_{0}^{2}$.

In a coordinate system fixed in the laboratory frame, the absolute fluid velocity $\boldsymbol{U}$ and the pressure $P$ satisfy the Navier-Stokes equations

$$
\begin{gathered}
\boldsymbol{\nabla} \cdot \boldsymbol{U}=0, \\
\frac{1}{\operatorname{Re}} \nabla \cdot \boldsymbol{\Sigma}=\frac{\partial \boldsymbol{U}}{\partial t}+\nabla \frac{\boldsymbol{U} \cdot \boldsymbol{U}}{2}+\boldsymbol{\omega} \times \boldsymbol{U},
\end{gathered}
$$

where $\boldsymbol{\Sigma}=-\operatorname{RePI}+\boldsymbol{\tau}$ is the stress tensor, whose viscous part is $\boldsymbol{\tau}=\boldsymbol{\nabla} \boldsymbol{U}+{ }^{\mathrm{T}} \boldsymbol{\nabla} \boldsymbol{U}$, and $\boldsymbol{\omega}=\boldsymbol{\nabla} \times \boldsymbol{U}$ is the vorticity. Let $\boldsymbol{r}$ denote the local position from the body centre of mass and $\boldsymbol{n}$ the unit normal pointing towards the fluid interior. Since any point of the body moves with the velocity $\boldsymbol{W}=\boldsymbol{V}+\boldsymbol{\Omega} \times \boldsymbol{r}$, the fluid velocity $\boldsymbol{U}$ at the body surface $S_{B}$ obeys the kinematic condition

$$
\boldsymbol{U} \cdot \boldsymbol{n}=\boldsymbol{W} \cdot \boldsymbol{n}=\boldsymbol{V} \cdot \boldsymbol{n}+\boldsymbol{\Omega} \cdot(\boldsymbol{r} \times \boldsymbol{n}),
$$

together with either the shear-free condition

$$
\boldsymbol{n} \times(\boldsymbol{\Sigma} \cdot \boldsymbol{n})=\mathbf{0}
$$

or the no-slip condition

$$
\boldsymbol{n} \times \boldsymbol{U}=\boldsymbol{n} \times \boldsymbol{W} .
$$

A fixed wall $S_{W}$ extending to infinity may partially bound the flow domain or may entirely enclose the fluid. The no-slip condition generally holds on $S_{W}$, so that

$$
\boldsymbol{U} \cdot \boldsymbol{n}=0
$$

and

$$
\boldsymbol{n} \times \boldsymbol{U}=\mathbf{0} .
$$

Finally, the velocity disturbance vanishes on the outer surface $S_{\infty}$ located at large distance from the body, so that

$$
\boldsymbol{U} \rightarrow \mathbf{0} \text { on } S_{\infty}
$$

In what follows we also need the auxiliary irrotational velocity field $\hat{U}$ and the associated pressure field $\hat{P}$ corresponding to those induced by the body as it translates with a unit velocity $\hat{\boldsymbol{i}}$ along some arbitrary fixed direction and rotates at a unit rate $\hat{j}$ 


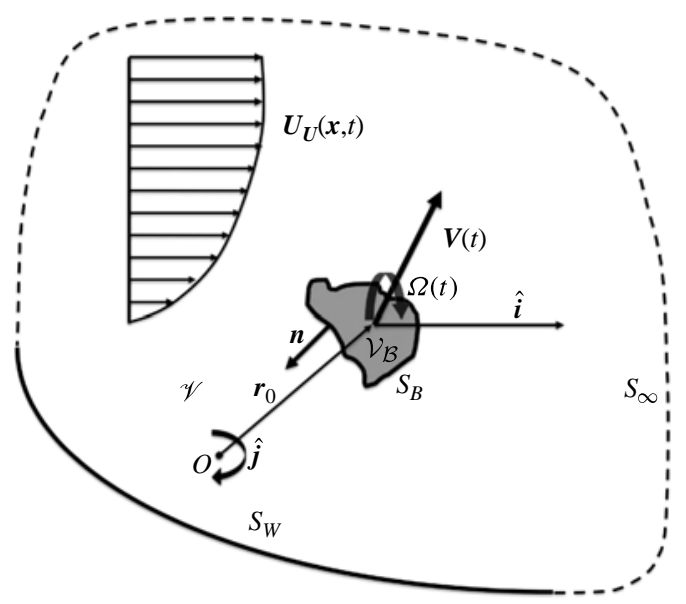

FIGURE 1. Sketch of the general flow configuration. The undisturbed velocity field $\boldsymbol{U}_{U}(\boldsymbol{x}, t)$ is non-zero only in $\S 3$. In the auxiliary problem, the body translates with a unit velocity along the $\hat{i}$ direction and rotates with a unit rotation rate about the $\hat{j}$ axis, the fluid being at rest at infinity.

about an arbitrary fixed axis passing through the origin $O$ of the coordinate system. This auxiliary velocity field is governed by

$$
\begin{gathered}
\nabla \cdot \hat{\boldsymbol{U}}=0, \\
\frac{1}{R e} \nabla \cdot \hat{\boldsymbol{\Sigma}}=\frac{\partial \hat{\boldsymbol{U}}}{\partial t}+\nabla \frac{\hat{\boldsymbol{U}} \cdot \hat{\boldsymbol{U}}}{2},
\end{gathered}
$$

where $\hat{\boldsymbol{\Sigma}}=-\operatorname{Re} \hat{P} \boldsymbol{I}+\hat{\boldsymbol{\tau}}$, with $\hat{\boldsymbol{\tau}}=\nabla \hat{\boldsymbol{U}}+{ }^{\mathrm{T}} \nabla \hat{\boldsymbol{U}}$. We may then introduce the velocity potential $\hat{\phi}$ such that $\hat{\boldsymbol{U}}=\nabla \hat{\phi}$. In cases in which the flow domain is not simply connected, $\hat{\phi}$ is made unique by requiring that there is no circulation around the body. The momentum balance for this viscous potential flow yields two separate conditions valid throughout the flow domain $\mathscr{V}$, namely

$$
\nabla \cdot \hat{\boldsymbol{\tau}}=-\nabla \times(\nabla \times \hat{\boldsymbol{U}})=\mathbf{0}
$$

and the Bernoulli integral

$$
\frac{\partial \hat{\phi}}{\partial t}+\frac{\hat{\boldsymbol{U}} \cdot \hat{\boldsymbol{U}}}{2}+\hat{P}=C(t),
$$

where $C$ is a constant. As will be seen later, the condition (2.11), which expresses the property of zero flux of viscous stresses in an irrotational incompressible flow, plays a key role in the derivation of the theorem.

Owing to the irrotationality constraint, $\hat{\boldsymbol{U}}$ only satisfies the kinematic condition at the body surface $S_{B}$ and on the wall $S_{W}$. Hence denoting with $\boldsymbol{r}_{0}(t)$ the position of the body centre of mass with respect to the origin of the coordinate system and defining $\hat{\boldsymbol{W}}=\hat{\boldsymbol{i}}+\hat{\boldsymbol{j}} \times\left(\boldsymbol{r}+\boldsymbol{r}_{0}\right)=\hat{\boldsymbol{I}}+\hat{\boldsymbol{j}} \times \boldsymbol{r}$ with $\hat{\boldsymbol{I}}=\hat{\boldsymbol{i}}+\hat{\boldsymbol{j}} \times \boldsymbol{r}_{0}$, one has

$$
\begin{gathered}
\hat{\boldsymbol{U}} \cdot \boldsymbol{n}=\frac{\partial \hat{\phi}}{\partial n}=\hat{\boldsymbol{W}} \cdot \boldsymbol{n} \quad \text { on } S_{B}, \\
\hat{\boldsymbol{U}} \cdot \boldsymbol{n}=0 \quad \text { on } S_{W},
\end{gathered}
$$


while at large distances one has

$$
\hat{\boldsymbol{U}} \rightarrow 0 \text { on } S_{\infty}
$$

\subsection{Derivation of the theorem}

Duplicating the classical procedure employed to establish reciprocal theorems, we start by forming the difference between the dot product of (2.2) and $\hat{\boldsymbol{U}}$ and that of (2.11) and $\boldsymbol{U}$, so as to eliminate the pseudo-dissipation rate $2 R e^{-1} \boldsymbol{\tau}: \hat{\boldsymbol{\tau}}$. Then, integrating over $\mathscr{V}$, making use of the divergence theorem and of the divergence-free condition yields

$$
\begin{aligned}
& \int_{S_{B} \cup S_{W}} \hat{\boldsymbol{U}} \cdot\left[\frac{1}{\operatorname{Re}} \boldsymbol{\Sigma} \cdot \boldsymbol{n}-\frac{1}{2}(\boldsymbol{U} \cdot \boldsymbol{U}) \boldsymbol{n}\right] \mathrm{d} S \\
& =\frac{1}{R e} \int_{S_{B}} \boldsymbol{U} \cdot(\hat{\boldsymbol{\tau}} \cdot \boldsymbol{n}) \mathrm{d} S-\int_{\mathscr{V}}\left[\frac{\partial \boldsymbol{U}}{\partial t}+\boldsymbol{\omega} \times \boldsymbol{U}\right] \cdot \hat{\boldsymbol{U}} \mathrm{d} V,
\end{aligned}
$$

where the no-slip condition (2.7) has been assumed to hold on $S_{W}$. Contributions from $S_{\infty}$ vanish as $r=\|\boldsymbol{r}\| \rightarrow \infty$, thanks to the $r^{-3}$ decay of $\hat{\boldsymbol{U}}$. Similarly, the volume integral on the right-hand side of (2.16) is convergent, provided $\boldsymbol{U}$ decays as $r^{-n}$ with $n>0$ (and hence $\omega$ decays as $r^{-n-1}$ ) in the wake of the body.

Conditions (2.13) and (2.14) allow the left-hand side of (2.16) to be transformed into

$$
\begin{aligned}
\int_{S_{B} \cup S_{W}} \hat{\boldsymbol{U}} \cdot\left\{\frac{1}{R \boldsymbol{e}} \boldsymbol{\Sigma} \cdot \boldsymbol{n}-\frac{1}{2}(\boldsymbol{U} \cdot \boldsymbol{U}) \boldsymbol{n}\right\} \mathrm{d} S \\
=\hat{\boldsymbol{I}} \cdot \boldsymbol{F}+\hat{\boldsymbol{j}} \cdot \boldsymbol{\Gamma}-\int_{S_{B}} \frac{1}{2}(\boldsymbol{U} \cdot \boldsymbol{U}) \hat{\boldsymbol{W}} \cdot \boldsymbol{n} \mathrm{d} S \\
\quad+\frac{1}{\operatorname{Re}} \int_{S_{W}} \hat{\boldsymbol{U}}_{S} \cdot(\boldsymbol{\tau} \cdot \boldsymbol{n})_{S} \mathrm{~d} S+\frac{1}{R \boldsymbol{e}} \int_{S_{B}}(\hat{\boldsymbol{U}}-\hat{\boldsymbol{W}})_{S} \cdot(\boldsymbol{\tau} \cdot \boldsymbol{n})_{S} \mathrm{~d} S,
\end{aligned}
$$

where $\boldsymbol{F}=R e^{-1} \int_{S_{B}} \boldsymbol{\Sigma} \cdot \boldsymbol{n} \mathrm{d} S$ and $\boldsymbol{\Gamma}=R \boldsymbol{e}^{-1} \int_{S_{B}} \boldsymbol{r} \times(\boldsymbol{\Sigma} \cdot \boldsymbol{n}) \mathrm{d} S$ are the hydrodynamic force and torque experienced by the body, respectively, and the subscript index $S$ denotes the tangential component of the corresponding vector (for instance, $\left.\hat{\boldsymbol{U}}_{S}=\boldsymbol{n} \times(\hat{\boldsymbol{U}} \times \boldsymbol{n})\right)$. The last term in (2.17) vanishes for a bubble submitted to the shear-free condition (2.4).

A series of manipulations based on the kinematic results established in appendix A and on the Stokes theorem are then required to make vorticity apparent in the viscous contributions on $S_{B}$ in (2.16) and (2.17), irrespective of the dynamic boundary condition that holds at the body surface. Similarly, the volume contribution involving the acceleration $\partial \boldsymbol{U} / \partial t$ in (2.16) may be transformed to make added-mass effects apparent by employing Leibnitz's rule and the divergence theorem. The details of these transformations are given in appendix B. Making use of (B 1) and (B 7), (2.16) finally yields the desired 'reciprocal' theorem in the form

$$
\begin{aligned}
\hat{\boldsymbol{I}} \cdot \boldsymbol{F}+\hat{\boldsymbol{j}} \cdot \boldsymbol{\Gamma}= & \frac{\mathrm{d}_{W}}{\mathrm{~d} t} \int_{S_{B}} \hat{\boldsymbol{\phi}} \boldsymbol{W} \cdot \boldsymbol{n} \mathrm{d} S+\int_{S_{B}}\left\{\left(\frac{1}{2} \boldsymbol{U} \cdot \boldsymbol{U}\right) \hat{\boldsymbol{W}}-\frac{\mathrm{D} \hat{\phi}}{\mathrm{D} t} \boldsymbol{W}\right\} \cdot \boldsymbol{n} \mathrm{d} S \\
& -\frac{1}{\operatorname{Re}} \int_{S_{B}}\{(\hat{\boldsymbol{U}}-\hat{\boldsymbol{W}}) \times(\boldsymbol{\omega}-2 \boldsymbol{\Omega})\} \cdot \boldsymbol{n} \mathrm{d} S-\frac{2}{\operatorname{Re}} \hat{\boldsymbol{j}} \cdot \int_{S_{B}} \boldsymbol{n} \times(\boldsymbol{U}-\boldsymbol{W}) \mathrm{d} S \\
& -\int_{\mathscr{V}}(\boldsymbol{\omega} \times \boldsymbol{U}) \cdot \hat{\boldsymbol{U}} \mathrm{d} V-\frac{1}{R e} \int_{S_{W}}(\hat{\boldsymbol{U}} \times \boldsymbol{\omega}) \cdot \boldsymbol{n} \mathrm{d} S
\end{aligned}
$$


where $\mathrm{d}_{W} / \mathrm{d} t$ is the time derivative following the motion of the body centre of mass, $\mathrm{D} / \mathrm{D} t=\partial / \partial t+\boldsymbol{U} \cdot \boldsymbol{\nabla}$ is the material derivative, and (A 6) has been employed to express the contribution of the shear stress $(\boldsymbol{\tau} \cdot \boldsymbol{n})_{S}$ on $S_{W}$ in terms of vorticity. All the terms in (2.18) have a straightforward physical interpretation, except for the second term on the right-hand side. However, another series of transformations detailed in appendix $\mathrm{C}$ and involving Green's second identity and repeated use of the divergence theorem may be applied to that term. In the course of these transformations, it is natural to introduce the bound vorticity $\boldsymbol{\omega}_{B}=\boldsymbol{n} \times(\boldsymbol{U}-\nabla \Phi) \delta_{B}, \delta_{B}$ being the Dirac function, which is zero everywhere except on $S_{B}$. This bound vorticity is due to the difference at the body surface between the tangential component of the actual velocity field $\boldsymbol{U}$ and that of the virtual irrotational velocity field $\nabla \Phi$ that satisfies the same kinematic boundary conditions (2.3) and (2.6) on $S_{B}$ and $S_{W}$. Then, provided the decay of the flow disturbance in the far field is fast enough (see below), use can be made of (C9) so as to write (2.18) in the physically clearer form

$$
\begin{aligned}
\hat{\boldsymbol{I}} \cdot \boldsymbol{F}+\hat{\boldsymbol{j}} \cdot \boldsymbol{\Gamma}= & \frac{\mathrm{d}_{W}}{\mathrm{~d} t} \int_{S_{B}} \hat{\phi} \boldsymbol{W} \cdot \boldsymbol{n} \mathrm{d} S+\int_{\mathscr{V}}\left\{\left(\boldsymbol{\omega}+\boldsymbol{\omega}_{B}\right) \times \boldsymbol{U}\right\} \cdot(\hat{\boldsymbol{W}}-\hat{\boldsymbol{U}}) \mathrm{d} V \\
& -\frac{1}{\operatorname{Re}} \int_{S_{B}}\{[(\hat{\boldsymbol{U}}-\hat{\boldsymbol{W}}) \times(\boldsymbol{\omega}-2 \boldsymbol{\Omega})] \cdot \boldsymbol{n}+2 \hat{\boldsymbol{j}} \cdot[\boldsymbol{n} \times(\boldsymbol{U}-\boldsymbol{W})]\} \mathrm{d} S \\
& -\int_{S_{W}}\left\{\frac{1}{2}(\boldsymbol{U} \cdot \boldsymbol{U}) \hat{\boldsymbol{W}} \cdot \boldsymbol{n}+\frac{1}{R e}(\hat{\boldsymbol{U}} \times \boldsymbol{\omega}) \cdot \boldsymbol{n}\right\} \mathrm{d} S .
\end{aligned}
$$

A final step may be carried out to recast the first integral on the right-hand side of (2.19) in terms of elementary classical added-mass contributions. Thanks to the linear dependence of $\hat{\boldsymbol{\phi}}$ with respect to $\hat{\boldsymbol{I}}$ and $\hat{\boldsymbol{j}}$ emphasized by (2.13), one may set

$$
\hat{\phi}=\boldsymbol{\Psi}_{T} \cdot \hat{\boldsymbol{I}}+\boldsymbol{\Psi}_{R} \cdot \hat{\boldsymbol{j}},
$$

where the two vectors $\boldsymbol{\Psi}_{T}$ and $\boldsymbol{\Psi}_{R}$ are harmonic functions that, according to (2.13) and (2.14), satisfy

$$
\begin{aligned}
\frac{\partial \boldsymbol{\Psi}_{T}}{\partial n}=\boldsymbol{n} & \text { and } \quad \frac{\partial \boldsymbol{\Psi}_{R}}{\partial n}=\boldsymbol{r} \times \boldsymbol{n} \quad \text { on } S_{B}, \\
\frac{\partial \boldsymbol{\Psi}_{T}}{\partial n}=0 & \text { and } \quad \frac{\partial \boldsymbol{\Psi}_{R}}{\partial n}=0 \quad \text { on } S_{W} .
\end{aligned}
$$

Introducing the usual second-order added-mass tensors $\boldsymbol{A}, \boldsymbol{B}, \boldsymbol{C}$ and $\boldsymbol{D}$ such that

$$
\begin{array}{lll}
\boldsymbol{A}={ }^{\mathrm{T}} \boldsymbol{A}=-\int_{S_{B}} \boldsymbol{\Psi}_{T} \frac{\partial \boldsymbol{\Psi}_{T}}{\partial n} \mathrm{~d} S, & \boldsymbol{B}={ }^{\mathrm{T}} \boldsymbol{C}=-\int_{S_{B}} \boldsymbol{\Psi}_{T} \frac{\partial \boldsymbol{\Psi}_{R}}{\partial n} \mathrm{~d} S, \\
\boldsymbol{C}={ }^{\mathrm{T}} \boldsymbol{B}=-\int_{S_{B}} \boldsymbol{\Psi}_{R} \frac{\partial \boldsymbol{\Psi}_{T}}{\partial n} \mathrm{~d} S, & \boldsymbol{D}={ }^{\mathrm{T}} \boldsymbol{D}=-\int_{S_{B}} \boldsymbol{\Psi}_{R} \frac{\partial \boldsymbol{\Psi}_{R}}{\partial n} \mathrm{~d} S,
\end{array}
$$

one has

$$
-\int_{S_{B}} \hat{\phi} \boldsymbol{W} \cdot \boldsymbol{n} \mathrm{d} S=\hat{\boldsymbol{I}} \cdot(\boldsymbol{A} \cdot \boldsymbol{V}+\boldsymbol{B} \cdot \boldsymbol{\Omega})+\hat{\boldsymbol{j}} \cdot(\boldsymbol{C} \cdot \boldsymbol{V}+\boldsymbol{D} \cdot \boldsymbol{\Omega}) .
$$

Keeping in mind that $\hat{\boldsymbol{I}}=\hat{\boldsymbol{i}}+\hat{\boldsymbol{j}} \times \boldsymbol{r}_{0}$, noting that $\mathrm{d}_{W} \boldsymbol{r}_{0} / \mathrm{d} t=\boldsymbol{V}$ and that the time derivative of any time-dependent vector $\boldsymbol{Q}$ in the laboratory frame and that in the reference frame rotating with the body, say $\mathrm{d}_{\Omega} / \mathrm{d} t$, are related by $\mathrm{d}_{\Omega} \boldsymbol{Q} / \mathrm{d} t=\mathrm{d}_{W} \boldsymbol{Q} / \mathrm{d} t-\boldsymbol{\Omega} \times \boldsymbol{Q}$, one 
obtains in the most general case

$$
\begin{aligned}
-\frac{\mathrm{d}_{W}}{\mathrm{~d} t} \int_{S_{B}} \hat{\phi} \boldsymbol{W} \cdot \boldsymbol{n} \mathrm{d} S= & \hat{\boldsymbol{I}} \cdot\left\{\frac{\mathrm{d}_{\Omega}(\boldsymbol{A} \cdot \boldsymbol{V})}{\mathrm{d} t}+\frac{\mathrm{d}_{\Omega}(\boldsymbol{B} \cdot \boldsymbol{\Omega})}{\mathrm{d} t}+\boldsymbol{\Omega} \times(\boldsymbol{A} \cdot \boldsymbol{V}+\boldsymbol{B} \cdot \boldsymbol{\Omega})\right\} \\
& +\hat{\boldsymbol{j}} \cdot\left\{\boldsymbol{V} \times(\boldsymbol{A} \cdot \boldsymbol{V}+\boldsymbol{B} \cdot \boldsymbol{\Omega})+\frac{\mathrm{d}_{\Omega}(\boldsymbol{C} \cdot \boldsymbol{V})}{\mathrm{d} t}+\frac{\mathrm{d}_{\Omega}(\boldsymbol{D} \cdot \boldsymbol{\Omega})}{\mathrm{d} t}\right. \\
& +\boldsymbol{\Omega} \times(\boldsymbol{C} \cdot \boldsymbol{V}+\boldsymbol{D} \cdot \boldsymbol{\Omega})\},
\end{aligned}
$$

where the derivatives $\mathrm{d}_{\Omega} \boldsymbol{A} / \mathrm{d} t=\boldsymbol{V} \cdot \nabla \boldsymbol{A}$ etc. are non-zero only if the body moves in the presence of a wall and its velocity $\boldsymbol{V}$ has a non-zero component perpendicular to that wall.

\subsection{The various contributions to the force and torque}

In the specific case of a rigid body subject to a no-slip condition and moving in an unbounded fluid, (2.19) is equivalent to the separate expressions (2.24) for the force and (3.7) for the torque obtained by Howe ( $\square$ ). When the body moves in a bounded fluid, the expression for the force in (2.18) is also equivalent to equation (6) of Grotta Ragazzo \& Tabak ( $\square)$.

The interpretation of all the contributions on the right-hand side of (2.19) is clear. The first term, which is entirely determined by the auxiliary potential $\hat{\phi}$ and the kinematic boundary condition at the body surface, represents the so-called added-mass loads. This contribution is independent of any effects of both vorticity and viscosity (Mougin \& Magnaudet $\square$ ). Consequently, it is not influenced by any possible separation of the flow past the body, although this is sometimes erroneously thought to be the case. Note that this is the only contribution to the force and torque involving the current fluid acceleration. The second term represents the contribution resulting from the presence of vorticity in the body of the fluid (the so-called free vorticity), supplemented by that of the bound vorticity on $S_{B}$. Note that, on $S_{B}, \hat{\boldsymbol{W}}-\hat{\boldsymbol{U}}$ is tangent to the body surface owing to the kinematic condition (2.3). Therefore, in the vicinity of the body, the leading contribution to this volume integral comes from fluid elements for which the cross product between $\boldsymbol{\omega}$ and $\boldsymbol{U}$ has a non-zero component tangent to $S_{B}$. This remark helps one to appreciate correctly the role of boundary layers in the total force. The third term is due to the dynamic boundary condition at the body surface. Hence it represents the viscous loads produced by the surface vorticity (often referred to as skin friction when the no-slip condition applies). If the body is submitted to a shear-free condition, the slip between the body and the fluid results in an additional viscous contribution to the torque that was not present in Howe's equation (3.7), nor in equation (17) of Grotta Ragazzo \& Tabak ( $\square$ ), since both groups only considered the torque balance on a body obeying a no-slip condition. The fourth term results from the presence of the wall and generally comprises an inertial and a viscous contribution. The former, which is non-zero only if the fluid is able to slip along the wall (e.g. when $S_{W}$ is a symmetry plane), does not contribute to the force in the direction parallel to the wall, since $\hat{\boldsymbol{W}} \cdot \boldsymbol{n}=\hat{\boldsymbol{I}} \cdot \boldsymbol{n}=0$ in that case. In contrast, whatever the direction of the body motion, this term results in an additional non-zero force normal to the wall and in a torque. When the no-slip condition holds on $S_{W}$, this inertial contribution is still physically present but takes a different form in (2.19); indeed it may then be shown that it results from the near-wall contribution to the volume integral $\int_{\mathscr{V}}(\boldsymbol{\omega} \times \boldsymbol{U}) \cdot(\hat{\boldsymbol{W}}-\hat{\boldsymbol{U}}) \mathrm{d} V$ (this may be established by splitting $\boldsymbol{U}$ into an outer 
and an inner contribution, the latter being due to the no-slip condition on $S_{W}$, and using arguments similar to those employed later at the end of $\S 4.1$ ).

The viscous contribution on $S_{W}$ vanishes if the wall reduces to a symmetry plane since the vorticity may only have a normal component on such a surface. In that case there is also an extra viscous term $\operatorname{Re}^{-1} \int_{S_{W}} \boldsymbol{U}_{S} \cdot(\hat{\boldsymbol{\tau}} \cdot \boldsymbol{n})_{S} \mathrm{~d} S$ in (2.16), but this contribution also vanishes since the tangential stress $(\hat{\boldsymbol{\tau}} \cdot \boldsymbol{n})_{S}$ is zero by virtue of (A 9). From the above remarks it can be concluded in particular that a symmetry plane does not provide any contribution to the drag force, be it viscous or inertial, when the body translates parallel to it. Thus D'Alembert's paradox still holds for such a body in steady motion in a viscous potential flow.

\subsection{Influence of the flow behaviour at large distance from the body}

Some comments about the conditions that the flow disturbance must fulfil on $S_{\infty}$ for the transformation from (2.18) into (2.19) to be valid seem in order. From a theoretical point of view, the compactness of the vorticity distribution, i.e. the fact that $\omega$ is vanishingly small outside a finite region of the control volume $\mathscr{V}$ over which integration is carried out, suffices to guarantee that all integrals on $S_{\infty}$ vanish when the surface recedes to infinity. In cases in which $\omega$ is not naturally compact within $\mathscr{V}$, the difficulty is classically by-passed by defining an extended vorticity distribution within the body volume $\mathscr{V}_{B}$ of surface $S_{B}$ and beyond the outer surface $S_{\infty}$ of $\mathscr{V}$ so as to reconnect properly the vortex tubes that cross $S_{B}$ and $S_{\infty}$. Integration may then be performed within the extended control volume $V^{+}$of outer surface $S^{+}$in which all vortex tubes are closed and vorticity is vanishingly small outside some finite subvolume $V_{\omega}$ (Batchelor $\square$; Saffman $\square$ ). Then the velocity field is irrotational within $V^{+}-V_{\omega}$ and the Biot-Savart law implies that it decays like $r^{-3}$ with the distance $r$ to the body at large distances from $V_{\omega}$, which guarantees the convergence of the various integrals on $V^{+}$and $S^{+}$. Therefore (2.19) is always a valid theoretical result.

The situation may be different if one is to use the present results to evaluate the various contributions to the force or torque by post-processing direct numerical simulation (DNS) data, as has been done in several studies (Chang \& Chern $\square$; Protas et al. $\square$; Pan \& Chew $\square$; Chang et al. $\square$ ). In DNS it is generally not possible to use a control volume satisfying the above properties. Although the computational domain $V_{C}$ extends far downstream from the body, vortices are generally shed across part of the surface $S_{D}$ that bounds it externally. Hence, within some parts of $V_{C}$, velocities decay much more slowly than $r^{-3}$ and may remain significant on parts of $S_{D}$. For instance, if an axisymmetric (Oseen) wake crosses $S_{D}$ normally, the normal velocity on this downstream surface is of $O\left(r^{-1}\right)$ within the wake and of $O\left(r^{-2}\right)$ outside it (Batchelor $\left.\square\right)$. Under such conditions, several technical steps used in the transformation from (2.18) to (2.19) may no longer be legitimate, as they may result in finite or even diverging contributions on $S_{D}$ and diverging volume integrals over $V_{C}$ (see the discussion in appendix $\mathrm{C}$ after $(\mathrm{C} 7)$ ). This is why it is then necessary to examine carefully how the actual velocity field decays at large $r$ before trying to compare computational results with (2.19). In summary, the main message to be kept in mind is that the form (2.19) of the 'reciprocal' theorem is certainly not always appropriate to compare DNS results with theoretical predictions, whereas the form (2.18) remains valid whatever the decay rate of the velocity disturbance in the far field. 


\section{A body moving in a non-uniform flow}

\subsection{The generalized form of the theorem}

It is obviously of great interest to examine how the above theorem may be extended to the general case of a body moving in an ambient non-uniform flow. The determination of forces and torques acting on bodies immersed in such flows has been a subject of active research since the pioneering works of Taylor $(\square)$ and Tollmien $(\square)$, who performed experiments on bodies of various shapes held fixed in converging, diverging or curved channels. Using an energy method, they also derived approximate expressions for the inviscid force and torque acting on non-rotating bodies placed in an irrotational weakly non-uniform stream. Since then, the problem has been reconsidered in greater generality by Galper \& Miloh $(\square, \square)$ in the case of rotating bodies of arbitrary shape moving in an irrotational linear flow field. Obviously, the case of ambient non-uniform flows with non-zero vorticity poses much greater difficulties. Combining a direct evaluation of the force in the case of a two-dimensional circular cylinder with an asymptotic estimate of leading-order force contributions in the case of a sphere (which in particular makes use of the prediction for the stationary inviscid shear-induced lift force obtained by Auton $(\square)$ ), Auton, Hunt \& Prud'homme ( $\square$ ) derived an expression for the inviscid hydrodynamic force acting on point-symmetric bodies moving in a weakly unsteady, slowly varying, linear flow. The problem was revisited by Miloh ( $\square$ ), who obtained expressions for the inviscid force and torque acting on rotating and translating bodies of arbitrary shape moving in a general linear flow field, with no restriction on the relative magnitude of the inhomogeneity, nor on that of unsteady effects. However Miloh's results were obtained under the highly restrictive assumption that the vorticity disturbance due to the distortion of the ambient vorticity by the body remains negligibly small, which prevents his results from being applicable to three-dimensional bodies, except at very short time after they have been introduced into the flow. The purpose of the present section is to extend the 'reciprocal' theorem derived in the previous section to such non-uniform situations so as to remove the limitations of the previous studies. The results available in the aforementioned literature should then be recovered as particular cases corresponding to the inviscid limit and to an unbounded flow domain.

To this end, let us consider the undisturbed incompressible flow whose velocity and pressure fields are $\boldsymbol{U}_{U}\left(\boldsymbol{r}+\boldsymbol{r}_{0}, t\right)$ and $P_{U}\left(\boldsymbol{r}+\boldsymbol{r}_{0}, t\right)$, respectively. If the flow domain is partially bounded by a wall, $\boldsymbol{U}_{U}$ satisfies the corresponding boundary conditions on $S_{W}$. In particular, one always has $\boldsymbol{U}_{U} \cdot \boldsymbol{n}=0$ on $S_{W}$. It is then suitable to work with the flow disturbance $\tilde{\boldsymbol{U}}=\boldsymbol{U}-\boldsymbol{U}_{U}$, which obeys

$$
\begin{aligned}
& \nabla \cdot \tilde{\boldsymbol{U}}=0, \\
& \frac{1}{\operatorname{Re}} \nabla \cdot \tilde{\boldsymbol{\Sigma}}=\left\{\frac{\partial}{\partial t}+\boldsymbol{U}_{U} \cdot \boldsymbol{\nabla}\right\} \tilde{\boldsymbol{U}}+\tilde{\boldsymbol{\omega}} \times \tilde{\boldsymbol{U}}+\frac{1}{2} \nabla(\tilde{\boldsymbol{U}} \cdot \tilde{\boldsymbol{U}})+\tilde{\boldsymbol{U}} \cdot \nabla \boldsymbol{U}_{U}, \\
& \tilde{\boldsymbol{U}} \cdot \boldsymbol{n}=0 \quad \text { and } \quad \tilde{\boldsymbol{U}} \times \boldsymbol{n}=\mathbf{0} \quad \text { on } S_{W}, \\
& \tilde{\boldsymbol{U}} \rightarrow \mathbf{0} \text { on } S_{\infty}
\end{aligned}
$$

where $\tilde{\boldsymbol{\Sigma}}=\boldsymbol{\Sigma}-\boldsymbol{\Sigma}_{U}$ and $\tilde{\boldsymbol{\omega}}=\boldsymbol{\omega}-\boldsymbol{\omega}_{U}$, with $\boldsymbol{\Sigma}_{U}$ and $\boldsymbol{\omega}_{U}$ denoting the stress tensor and the vorticity associated with the undisturbed flow, respectively. Except for the last term on the right-hand side, which is intrinsically new, (3.2) is formally identical to (2.2) provided the time derivative $\partial / \partial t$ is replaced by the Lagrangian derivative following the undisturbed flow $\mathrm{D}_{U} / \mathrm{D} t=\partial / \partial t+\boldsymbol{U}_{U} \cdot \boldsymbol{\nabla}$. Similarly, provided $\boldsymbol{W}$ is changed into $\tilde{\boldsymbol{W}}=\boldsymbol{W}-\boldsymbol{U}_{U}=\boldsymbol{V}-\boldsymbol{U}_{U}+\boldsymbol{\Omega} \times \boldsymbol{r}$, the kinematic boundary condition (2.3) still holds for 
the disturbance velocity $\tilde{\boldsymbol{U}}$. It is then straightforward to obtain the counterpart of (2.18) in the presence of a non-uniform underlying flow except for two points. First one needs to establish how terms involving $\mathrm{D}_{U} \tilde{\boldsymbol{U}} / \mathrm{D} t$ transform. Then one has to consider the contributions on $S_{\infty}$ encountered during the derivation and establish the conditions under which they are vanishingly small. Both points are addressed in appendix D. Combining the results of this appendix, especially (D2), with the above remarks allow most other steps leading to (2.18) to be readily repeated so as to obtain

$$
\begin{aligned}
\hat{\boldsymbol{I}} \cdot \boldsymbol{F}+\hat{\boldsymbol{j}} \cdot \boldsymbol{\Gamma}= & \hat{\boldsymbol{I}} \cdot \boldsymbol{F}_{U}+\hat{\boldsymbol{j}} \cdot \boldsymbol{\Gamma}_{U}+\frac{\mathrm{d}_{W}}{\mathrm{~d} t} \int_{S_{B}} \hat{\boldsymbol{\phi}} \tilde{\boldsymbol{W}} \cdot \boldsymbol{n} \mathrm{d} S-\int_{S_{B}} \frac{\mathrm{D} \hat{\boldsymbol{\phi}}}{\mathrm{D} t} \tilde{\boldsymbol{W}} \cdot \boldsymbol{n} \mathrm{d} S \\
& +\frac{1}{2} \int_{S_{B}}(\tilde{\boldsymbol{U}} \cdot \tilde{\boldsymbol{U}}) \hat{\boldsymbol{W}} \cdot \boldsymbol{n} \mathrm{d} S-\int_{\mathscr{V}}(\tilde{\boldsymbol{\omega}} \times \tilde{\boldsymbol{U}}) \cdot \hat{\boldsymbol{U}} \mathrm{d} V-2 \int_{\mathscr{V}}\left(\tilde{\boldsymbol{U}} \cdot \nabla \boldsymbol{U}_{U}\right) \cdot \hat{\boldsymbol{U}} \mathrm{d} V \\
& -\frac{1}{\operatorname{Re}} \int_{S_{B}}\{(\hat{\boldsymbol{U}}-\hat{\boldsymbol{W}}) \times(\boldsymbol{\omega}-2 \boldsymbol{\Omega})\} \cdot \boldsymbol{n} \mathrm{d} S-\frac{2}{\operatorname{Re}} \hat{\boldsymbol{j}} \cdot \int_{S_{B}} \boldsymbol{n} \times(\tilde{\boldsymbol{U}}-\tilde{\boldsymbol{W}}) \mathrm{d} S \\
& +\frac{1}{\operatorname{Re}} \int_{S_{B}}\left\{(\hat{\boldsymbol{U}}-\hat{\boldsymbol{W}})\left(\boldsymbol{\tau}_{U} \cdot \boldsymbol{n}\right)-\boldsymbol{U}_{U} \cdot(\hat{\boldsymbol{\tau}} \cdot \boldsymbol{n})\right\} \mathrm{d} S \\
& -\frac{1}{R e} \int_{S_{W}}(\hat{\boldsymbol{U}} \times \tilde{\boldsymbol{\omega}}) \cdot \boldsymbol{n} \mathrm{d} S
\end{aligned}
$$

where $\boldsymbol{F}_{U}=R e^{-1} \int_{S_{B}} \boldsymbol{\Sigma}_{U} \cdot \boldsymbol{n} \mathrm{d} S$ and $\boldsymbol{\Gamma}_{U}=R e^{-1} \int_{S_{B}} \boldsymbol{r} \times\left(\boldsymbol{\Sigma}_{U} \cdot \boldsymbol{n}\right) \mathrm{d} S$ are the hydrodynamic force and torque exerted by the undisturbed flow on the volume of fluid $\mathscr{V}_{B}$ enclosed by $S_{B}$, and $\boldsymbol{\tau}_{U}=R e^{-1}\left(\boldsymbol{\nabla} \boldsymbol{U}_{U}+{ }^{\mathrm{T}} \nabla \boldsymbol{U}_{U}\right)$.

Again, it is desirable to transform to fourth and fifth terms on the right-hand side of (3.5) to allow for a clearer physical interpretation of the result. The corresponding transformations are detailed in appendix E. Provided the required conditions hold for some of these transformations to be valid (see again the discussion in $\S 2.4$ ), (E 8) allows (3.5) to be finally recast in the form

$$
\begin{aligned}
\hat{\boldsymbol{I}} \cdot \boldsymbol{F}+\hat{\boldsymbol{j}} \cdot \boldsymbol{\Gamma}= & \hat{\boldsymbol{I}} \cdot \boldsymbol{F}_{U}+\hat{\boldsymbol{j}} \cdot \boldsymbol{\Gamma}_{U}+\frac{\mathrm{d}_{W}}{\mathrm{~d} t} \int_{S_{B}} \hat{\boldsymbol{\phi}} \tilde{\boldsymbol{W}} \cdot \boldsymbol{n} \mathrm{d} S-\int_{S_{B}} \tilde{\boldsymbol{\Phi}}\left(\hat{\boldsymbol{j}} \times \boldsymbol{U}_{U}\right) \cdot \boldsymbol{n} \mathrm{d} S \\
& +\int_{S_{B}} \tilde{\boldsymbol{\Phi}} \hat{\boldsymbol{W}} \cdot \nabla \boldsymbol{U}_{U} \cdot \boldsymbol{n} \mathrm{d} S-\int_{\mathscr{V}}\left(\boldsymbol{\omega}_{U} \times \tilde{\boldsymbol{U}}\right) \cdot \hat{\boldsymbol{U}} \mathrm{d} V \\
& +\int_{\mathscr{V}}\left[\left(\tilde{\boldsymbol{\omega}}+\tilde{\boldsymbol{\omega}}_{B}\right) \times \tilde{\boldsymbol{U}}\right] \cdot(\hat{\boldsymbol{W}}-\hat{\boldsymbol{U}}) \mathrm{d} V \\
& -\frac{1}{\operatorname{Re}} \int_{S_{B}}\{(\hat{\boldsymbol{U}}-\hat{\boldsymbol{W}}) \times(\boldsymbol{\omega}-2 \boldsymbol{\Omega})\} \cdot \boldsymbol{n} \mathrm{d} S-\frac{2}{\operatorname{Re}} \hat{\boldsymbol{j}} \cdot \int_{S_{B}} \boldsymbol{n} \times(\tilde{\boldsymbol{U}}-\tilde{\boldsymbol{W}}) \mathrm{d} S \\
& +\frac{1}{\operatorname{Re}} \int_{S_{B}}\left\{(\hat{\boldsymbol{U}}-\hat{\boldsymbol{W}})\left(\boldsymbol{\tau}_{U} \cdot \boldsymbol{n}\right)-\boldsymbol{U}_{U} \cdot(\hat{\boldsymbol{\tau}} \cdot \boldsymbol{n})\right\} \mathrm{d} S \\
& -\int_{S_{W}}\left\{\frac{1}{2}(\tilde{\boldsymbol{U}} \cdot \tilde{\boldsymbol{U}}) \hat{\boldsymbol{W}}+\frac{1}{\operatorname{Re}} \hat{\boldsymbol{U}} \times \tilde{\boldsymbol{\omega}}\right\} \cdot \boldsymbol{n} \mathrm{d} S,
\end{aligned}
$$

where the velocity potential $\tilde{\Phi}$ satisfies $\boldsymbol{n} \cdot \nabla \tilde{\Phi}=\tilde{\boldsymbol{W}} \cdot \boldsymbol{n}$ on $S_{B}$ and $\boldsymbol{n} \cdot \nabla \tilde{\Phi}=0$ on $S_{W}$ and the bound vorticity is now defined as $\tilde{\boldsymbol{\omega}}_{B}=\boldsymbol{n} \times(\tilde{\boldsymbol{U}}-\nabla \tilde{\Phi}) \delta_{B}$. The first two terms on the right-hand side of (3.6) represent the body force and torque resulting from the possible acceleration of the undisturbed flow, while the sum of the next two terms is the added-mass contribution. Three extra terms result from the non-uniformity 
of the undisturbed flow. Two of them involving either $\boldsymbol{\nabla} \boldsymbol{U}_{U}$ or $\boldsymbol{\tau}_{U}$ appear in the form of surface integrals on $S_{B}$ and generally provide non-zero contributions even when the undisturbed flow is irrotational. There is also a third, contribution throughout $\mathscr{V}$ involving the possible non-zero vorticity $\omega_{U}$ of the undisturbed flow. Since the corresponding term takes the form of the cross product of $\omega_{U}$ by the relative velocity $\tilde{\boldsymbol{U}}$, it clearly provides a lift component to the force experienced by the body.

The form (3.6) of the 'reciprocal' theorem is the most general result derived in this paper, since it is valid for any undisturbed flow provided the conditions for the vanishing of contributions on $S_{\infty}$ established in appendix D are satisfied. Note that, if $\boldsymbol{U}_{U}$ is uniform, it may readily be proved that (3.6) satisfies Galilean invariance, as it should. This may be seen by noting that (B 3) implies that $\int_{S_{B}} \boldsymbol{U}_{U} \cdot(\hat{\boldsymbol{\tau}} \cdot \boldsymbol{n}) \mathrm{d} S=0$ when $\boldsymbol{U}_{U}$ is uniform and by using (2.25) and (2.26), which show that the added-mass contribution keeps the same form as in the case of a fluid at rest, except for the fact that $\boldsymbol{V}$ has to be replaced by $\tilde{\boldsymbol{V}}=\boldsymbol{V}-\boldsymbol{U}_{U}$ everywhere in (2.26).

\subsection{A body in a general linear flow}

To reveal the various effects of the undisturbed velocity gradients, we now specialize (3.6) to the widely encountered situation where the carrying flow can be considered linear, in which case

$$
\boldsymbol{U}_{U}\left(\boldsymbol{r}_{0}, \boldsymbol{r}, t\right)=\boldsymbol{U}_{0}\left(\boldsymbol{r}_{0}, t\right)+\boldsymbol{r} \cdot \nabla \boldsymbol{U}_{0}(t)=\boldsymbol{U}_{0}\left(\boldsymbol{r}_{0}, t\right)+\boldsymbol{r} \cdot \boldsymbol{S}_{0}(t)+\frac{1}{2} \boldsymbol{\omega}_{0}(t) \times \boldsymbol{r},
$$

where $\boldsymbol{r}_{0}$ still denotes the instantaneous position of the body centre of mass, and $\boldsymbol{S}_{0}$ and $\boldsymbol{\omega}_{0}$ are the constant-strain-rate tensor and vorticity of the undisturbed flow, respectively. Note that the presence of a wall imposes severe restrictions on the form of the linear flow that may exist in $\mathscr{V}$, owing to the kinematic boundary condition $\boldsymbol{U}_{U} \cdot \boldsymbol{n}=0$ on $S_{W}$. For instance, if $\boldsymbol{\omega}_{0}=\mathbf{0}$ and the undisturbed flow is a planar extensional flow, $S_{W}$ may be one of the two symmetry planes of that flow. Similarly, if $\boldsymbol{S}_{0}=\mathbf{0}$ and the flow configuration corresponds to a solid-body rotation in a circular cylinder, $S_{W}$ may be the lateral wall of the cylinder.

The kinematic boundary condition at the body surface now reads

$$
\tilde{\boldsymbol{W}} \cdot \boldsymbol{n}=\tilde{\boldsymbol{V}} \cdot \boldsymbol{n}+\tilde{\boldsymbol{\Omega}} \cdot(\boldsymbol{r} \times \boldsymbol{n})-\frac{1}{2} \boldsymbol{S}_{0}:(\boldsymbol{r n}+\boldsymbol{n r}),
$$

where $\tilde{\boldsymbol{V}}\left(\boldsymbol{r}_{0}, t\right)=\boldsymbol{V}-\boldsymbol{U}_{0}$ and $\tilde{\boldsymbol{\Omega}}(t)=\boldsymbol{\Omega}-\boldsymbol{\omega}_{0} / 2$. Hence the velocity potential $\tilde{\Phi}$ takes the form

$$
\tilde{\Phi}=\boldsymbol{\Psi}_{T} \cdot \tilde{\boldsymbol{V}}+\boldsymbol{\Psi}_{R} \cdot \tilde{\boldsymbol{\Omega}}-\boldsymbol{\Psi}_{S}: \boldsymbol{S}_{0},
$$

where $\boldsymbol{\Psi}_{T}$ and $\boldsymbol{\Psi}_{R}$ have been defined in $\S 2.2$ and $\boldsymbol{\Psi}_{S}$ is a symmetric second-order tensor satisfying

$$
\nabla^{2} \boldsymbol{\Psi}_{S}=\mathbf{0} \quad \text { in } V, \quad \frac{\partial \boldsymbol{\Psi}_{S}}{\partial n}=\frac{1}{2}(\boldsymbol{r n}+\boldsymbol{n r}) \quad \text { on } S_{B}, \quad \frac{\partial \boldsymbol{\Psi}_{S}}{\partial n}=\mathbf{0} \quad \text { on } S_{W} .
$$

From (3.8) we deduce that the integral $\int_{S_{B}} \hat{\phi} \tilde{\boldsymbol{W}} \cdot \boldsymbol{n} \mathrm{d} S$ may be expressed in the form

$$
\begin{aligned}
-\int_{S_{B}} \hat{\boldsymbol{\phi}} \tilde{\boldsymbol{W}} \cdot \boldsymbol{n} \mathrm{d} S= & \hat{\boldsymbol{I}} \cdot\left(\boldsymbol{A} \cdot \tilde{\boldsymbol{V}}+\boldsymbol{B} \cdot \tilde{\boldsymbol{\Omega}}-\boldsymbol{E}_{T}: \boldsymbol{S}_{0}\right) \\
& +\hat{\boldsymbol{j}} \cdot\left(\boldsymbol{C} \cdot \tilde{\boldsymbol{V}}+\boldsymbol{D} \cdot \tilde{\boldsymbol{\Omega}}-\boldsymbol{E}_{R}: \boldsymbol{S}_{0}\right)
\end{aligned}
$$


where $\boldsymbol{E}_{T}$ and $\boldsymbol{E}_{R}$ are two third-order tensors, respectively defined by

$$
\left.\begin{array}{c}
\boldsymbol{E}_{T}=\int_{S_{B}} \boldsymbol{\Psi}_{T} \frac{\partial \boldsymbol{\Psi}_{S}}{\partial n} \mathrm{~d} S=\int_{S_{B}} \frac{\partial \boldsymbol{\Psi}_{T}}{\partial n} \boldsymbol{\Psi}_{S} \mathrm{~d} S, \\
\boldsymbol{E}_{R}=\int_{S_{B}} \boldsymbol{\Psi}_{R} \frac{\partial \boldsymbol{\Psi}_{S}}{\partial n} \mathrm{~d} S=\int_{S_{B}} \frac{\partial \boldsymbol{\Psi}_{R}}{\partial n} \boldsymbol{\Psi}_{S} \mathrm{~d} S .
\end{array}\right\}
$$

The form (3.9) of the velocity potential makes it clear that quadratic contributions with respect to $\boldsymbol{\omega}_{0}$ and $\boldsymbol{S}_{0}$ are present in every inertial term of (3.6). However, to avoid too cumbersome formulae while capturing the leading effects of the inhomogeneity, we shall neglect these terms and consider only linear contributions. This simplification is based on the assumption that, in many situations of practical interest, $\left\|\boldsymbol{r} \cdot \boldsymbol{S}_{0}\right\| /\|\tilde{\boldsymbol{V}}\| \ll 1$ and $\left\|\boldsymbol{r} \times \omega_{0}\right\| /\|\tilde{\boldsymbol{V}}\| \ll 1$ for $\|\boldsymbol{r}\|=O(1)$, i.e. the typical length scale of the body is small compared to the characteristic length scale of the inhomogeneity. Quadratic terms have been considered in the inviscid approximation, either in the irrotational case by Galper \& Miloh ( $\square$ ) or in the short-time limit in rotational flows by Miloh ( $\square)$. A consequence of the above assumption is that $\omega_{0}$ must be considered independent of time from now on, since in a linear flow the evolution of the vorticity is governed by the Helmholtz equation $\mathrm{d} \boldsymbol{\omega}_{0} / \mathrm{d} t=\omega_{0} \cdot \boldsymbol{S}_{0}$.

With a linear undisturbed flow, the viscous contribution at the body surface in (3.6) may be simplified by recognizing that $\boldsymbol{\nabla} \cdot \boldsymbol{\tau}_{U}=\mathbf{0}$, a property implying that $\int_{S_{B}} \hat{\boldsymbol{W}} \cdot\left(\boldsymbol{\tau}_{U} \cdot \boldsymbol{n}\right) \mathrm{d} S=0$. Since $\hat{\boldsymbol{\tau}}$ is also divergence-free, Green's second identity implies that $\int_{S_{B} \cup S_{W}}\left\{\hat{\boldsymbol{U}} \cdot\left(\boldsymbol{\tau}_{U} \cdot \boldsymbol{n}\right)-\boldsymbol{U}_{U} \cdot(\hat{\boldsymbol{\tau}} \cdot \boldsymbol{n})\right\} \mathrm{d} S=0$, which allows the contribution of the undisturbed flow on $S_{B}$ to be transformed into a contribution on $S_{W}$. Applying the 'weak inhomogeneity' approximation defined above, (3.6) then becomes

$$
\begin{aligned}
\hat{\boldsymbol{I}} \cdot \boldsymbol{F}+\hat{\boldsymbol{j}} \cdot \boldsymbol{\Gamma}= & \hat{\boldsymbol{I}} \cdot \boldsymbol{F}_{0}+\hat{\boldsymbol{j}} \cdot \boldsymbol{\Gamma}_{0}+\frac{\mathrm{d}_{W}}{\mathrm{~d} t} \int_{S_{B}} \hat{\boldsymbol{\phi}} \tilde{\boldsymbol{W}} \cdot \boldsymbol{n} \mathrm{d} S-\left(\hat{\boldsymbol{j}} \times \boldsymbol{U}_{0}\right) \cdot \int_{S_{B}} \tilde{\boldsymbol{\Phi}} \boldsymbol{n} \mathrm{d} S \\
& +\int_{S_{B}} \tilde{\Phi}_{0}\left\{\hat{\boldsymbol{W}} \cdot \nabla \boldsymbol{U}_{0}-\hat{\boldsymbol{j}} \times\left(\boldsymbol{r} \cdot \nabla \boldsymbol{U}_{0}\right)\right\} \cdot \boldsymbol{n} \mathrm{d} S \\
& +\int_{\mathscr{V}}\left[\left(\tilde{\boldsymbol{\omega}}+\tilde{\boldsymbol{\omega}}_{B}\right) \times \tilde{\boldsymbol{U}}\right]_{0} \cdot(\hat{\boldsymbol{W}}-\hat{\boldsymbol{U}}) \mathrm{d} V-\int_{\mathscr{V}}\left(\boldsymbol{\omega}_{0} \times \tilde{\boldsymbol{U}}_{0}\right) \cdot \hat{\boldsymbol{U}} \mathrm{d} V \\
& -\frac{1}{\operatorname{Re}} \int_{S_{B}}\{(\hat{\boldsymbol{U}}-\hat{\boldsymbol{W}}) \times(\boldsymbol{\omega}-2 \boldsymbol{\Omega})\} \cdot \boldsymbol{n} \mathrm{d} S \\
& -\frac{2}{\operatorname{Re}} \hat{\boldsymbol{j}} \cdot \int_{S_{B}} \boldsymbol{n} \times(\tilde{\boldsymbol{U}}-\tilde{\boldsymbol{W}}) \mathrm{d} S \\
& -\int_{S_{W}}\left\{\frac{1}{2}(\tilde{\boldsymbol{U}} \cdot \tilde{\boldsymbol{U}})_{0} \hat{\boldsymbol{W}}+\frac{1}{R e} \hat{\boldsymbol{U}} \times \boldsymbol{\omega}\right\} \cdot \boldsymbol{n} \mathrm{d} S
\end{aligned}
$$

where $\tilde{\Phi}_{0}=\boldsymbol{\Psi}_{T} \cdot \tilde{\boldsymbol{V}}+\boldsymbol{\Psi}_{R} \cdot \boldsymbol{\Omega}$ and $\tilde{\boldsymbol{U}}_{0}=\boldsymbol{U}-\boldsymbol{U}_{0}$, and $\left[\left(\tilde{\boldsymbol{\omega}}+\tilde{\boldsymbol{\omega}}_{B}\right) \times \tilde{\boldsymbol{U}}\right]_{0}$ (respectively, $(\tilde{\boldsymbol{U}} \cdot \tilde{\boldsymbol{U}})_{0}$ ) denotes the corresponding cross product (respectively, dot product) linearized in the sense defined above. In (3.13), $\boldsymbol{F}_{0}=\left(\mathrm{D}_{0} \boldsymbol{U}_{0} / \mathrm{D} t\right) \mathscr{V}_{B}=\left\{\partial \boldsymbol{U}_{0} / \partial t+\boldsymbol{U}_{0} \cdot \boldsymbol{\nabla} \boldsymbol{U}_{0}\right\} \mathscr{V}_{B}$ is the linearized body force acting on the volume $\mathscr{V}_{B}$ and $\boldsymbol{\Gamma}_{0}=\int_{\mathscr{V}_{B}} \boldsymbol{r} \times\left(\boldsymbol{r} \cdot \mathrm{d} \boldsymbol{S}_{0} / \mathrm{d} t\right) \mathrm{d} V$ is the associated body torque, which is non-zero only if the undisturbed velocity gradients are time-dependent (the contribution $\int_{S_{B}}\left\{\boldsymbol{r} \times\left(\mathrm{D}_{0} \boldsymbol{U}_{0} / \mathrm{D} t\right)\right\} \mathrm{d} S$ to the torque is zero since $\boldsymbol{r}=\mathbf{0}$ corresponds to the body centre of mass). Note that the vortical part 
of the viscous contributions on both $S_{B}$ and $S_{W}$ involves the total vorticity $\omega$, not the vorticity disturbance $\tilde{\boldsymbol{\omega}}$.

To obtain a detailed view of the effects produced by the base flow, two more steps are necessary. First, all inertial contributions over the body surface (first three integrals on the right-hand side of (3.13)) must be expanded with respect to $\boldsymbol{S}_{0}, \boldsymbol{\omega}_{0}$ and $\mathrm{D}_{0} \boldsymbol{U}_{0} / \mathrm{D} t$. The result of this expansion is provided by $(\mathrm{F} 1)-(\mathrm{F} 4)$ in appendix $\mathrm{F}$. Then the volume integral involving $\omega_{0}$ must be transformed so as to disentangle contributions provided by the potential and vortical parts of the velocity disturbance $\tilde{\boldsymbol{U}}_{0}$. This transformation yields (F6), which reveals the existence of the surface contribution $\boldsymbol{\omega}_{0} \cdot \int_{S_{B}} \hat{\phi}\left(\boldsymbol{n} \times \nabla \tilde{\Phi}_{0}\right) \mathrm{d} S$, which only depends on the irrotational velocity field $\nabla \tilde{\Phi}_{0}$ and hence may, under certain conditions, be connected to the added-mass tensor.

Results (F 1)-(F 6) may finally be injected into (3.13) to obtain the final form of the 'reciprocal' theorem in a linear flow without any restriction on the body shape (but still in the weakly inhomogeneous approximation). As (F 2)-(F4) make clear, numerous couplings between the body rotation $\Omega$ and the strain rate $\boldsymbol{S}_{0}$ or the ambient vorticity $\omega_{0}$ contribute to the force and torque balances in the case of a general body shape. Those that subsist when the carrying flow is irrotational have been examined by Galper \& Miloh $(\square, \square)$ and the discussion will not be repeated here.

Fortunately, applications frequently involve bodies exhibiting geometrical symmetries that suppress most of these couplings and clarify the interpretation of the most important physical effects. Here we focus on the class of bodies exhibiting three orthogonal symmetry planes, which in particular encompasses axisymmetric bodies with fore-aft symmetry, among which are ellipsoids. However, even for such simple body geometries, the presence of a wall still maintains couplings between translational and rotational motions. Therefore, only two physical situations lead to significant simplifications. The first of these corresponds to the case of an unbounded flow domain or equivalently to a situation where the body moves at large distance from the wall so that wall effects can be ignored. The second corresponds to the situation where the body moves close to a plane wall without rotating, one of its symmetry planes being parallel to that wall. For such body shapes and flow conditions, the irrotational disturbance induced by the translational motion becomes uncoupled with those resulting from rotation (if any) and deformation. Hence $\boldsymbol{E}_{T}=\mathbf{0}$ and the only two added-mass tensors that subsist are $\boldsymbol{A}$ and $\boldsymbol{D}$. Moreover, as shown by (F7) and (F 8), all contributions to the torque involving the combined effect of the possible body rotation $\Omega$ and of the background vorticity $\omega_{0}$ are expressible via the third-order tensor $\boldsymbol{K}$ defined through the relation $\boldsymbol{D}=2(\boldsymbol{K} \cdot \boldsymbol{J}): \boldsymbol{\epsilon}$, where $\boldsymbol{J}=\int_{\mathscr{V}_{B}}\{(\boldsymbol{r} \cdot \boldsymbol{r}) \boldsymbol{I}-\boldsymbol{r} \boldsymbol{r}\} \mathrm{d} V$ is the inertia tensor and $\epsilon$ denotes the usual third-order alternating tensor.

Thus, making use of the various results established in appendix $F$, the form of (3.13) suitable for bodies exhibiting the symmetries defined above and moving in an unbounded flow domain is finally found to be

$$
\begin{aligned}
\hat{\boldsymbol{I}} \cdot \boldsymbol{F} & +\hat{\boldsymbol{j}} \cdot \boldsymbol{\Gamma} \\
= & \hat{\boldsymbol{I}} \cdot\left\{\frac{\mathrm{D}_{0} \boldsymbol{U}_{0}}{\mathrm{D} t} \mathscr{V}_{B}-\boldsymbol{A} \cdot\left(\frac{\mathrm{d} \boldsymbol{V}}{\mathrm{d} t}-\frac{\mathrm{D}_{0} \boldsymbol{U}_{0}}{\mathrm{D} t}-\boldsymbol{\Omega} \times \tilde{\boldsymbol{V}}\right)-\boldsymbol{\Omega} \times(\boldsymbol{A} \cdot \tilde{\boldsymbol{V}})\right. \\
& \left.+\left[\boldsymbol{A} \cdot \boldsymbol{S}_{0}-\boldsymbol{S}_{0} \cdot \boldsymbol{A}\right] \cdot \tilde{\boldsymbol{V}}+\left(\frac{\boldsymbol{I}}{2}+\frac{\boldsymbol{A}}{\mathscr{V}_{B}}\right) \cdot\left[\boldsymbol{\omega}_{0} \times(\boldsymbol{A} \cdot \tilde{\boldsymbol{V}})\right]-\frac{1}{2} \boldsymbol{A} \cdot\left(\tilde{\boldsymbol{V}} \times \boldsymbol{\omega}_{0}\right)\right\}
\end{aligned}
$$




$$
\begin{aligned}
& -\hat{\boldsymbol{j}} \cdot\left\{\tilde{\boldsymbol{V}} \times(\boldsymbol{A} \cdot \tilde{\boldsymbol{V}})+\boldsymbol{D} \cdot\left(\frac{\mathrm{d} \boldsymbol{\Omega}}{\mathrm{d} t}+\frac{1}{2} \boldsymbol{\Omega} \times \boldsymbol{\omega}_{0}\right)+\boldsymbol{\Omega} \times\left(\boldsymbol{D} \cdot\left(\boldsymbol{\Omega}-\frac{1}{2} \boldsymbol{\omega}_{0}\right)\right)\right. \\
& +\boldsymbol{E}_{R}: \frac{\mathrm{d} \boldsymbol{S}_{0}}{\mathrm{~d} t}-2 \boldsymbol{\epsilon}:\left(\boldsymbol{\Omega} \cdot \boldsymbol{E}_{R} \cdot \boldsymbol{S}_{0}\right)+2\left(\boldsymbol{E}_{R} \cdot \boldsymbol{S}_{0}\right):(\boldsymbol{\Omega} \cdot \boldsymbol{\epsilon})+\boldsymbol{\Omega} \times\left(\boldsymbol{E}_{R}: \boldsymbol{S}_{0}\right) \\
& \left.-4(\boldsymbol{K} \cdot \boldsymbol{J}) \cdot\left({ }^{\mathrm{T}} \boldsymbol{K} \cdot \boldsymbol{\Omega}\right):\left(\boldsymbol{\epsilon} \cdot \boldsymbol{\omega}_{0}\right)-(\boldsymbol{\Omega} \cdot \boldsymbol{K}) \cdot\left(\boldsymbol{J} \cdot \boldsymbol{\omega}_{0}\right)+\boldsymbol{J} \cdot\left(\boldsymbol{\omega}_{0} \cdot{ }^{\mathrm{T}} \boldsymbol{K} \cdot \boldsymbol{\Omega}\right)\right\} \\
& +\int_{\mathscr{V}}\left[\left(\tilde{\boldsymbol{\omega}}+\tilde{\boldsymbol{\omega}}_{B}\right) \times \tilde{\boldsymbol{U}}\right]_{0} \cdot(\hat{\boldsymbol{W}}-\hat{\boldsymbol{U}}) \mathrm{d} V-\int_{\mathscr{V}} \hat{\boldsymbol{\phi}} \boldsymbol{\omega}_{0} \cdot\left(\tilde{\boldsymbol{\omega}}+\tilde{\boldsymbol{\omega}}_{B}\right)_{0} \mathrm{~d} V \\
& -\frac{1}{\operatorname{Re}} \int_{S_{B}}\{(\hat{\boldsymbol{U}}-\hat{\boldsymbol{W}}) \times(\boldsymbol{\omega}-2 \boldsymbol{\Omega})\} \cdot \boldsymbol{n} \mathrm{d} S \\
& -\frac{2}{\operatorname{Re}} \hat{\boldsymbol{j}} \cdot \int_{S_{B}} \boldsymbol{n} \times(\tilde{\boldsymbol{U}}-\tilde{\boldsymbol{W}}) \mathrm{d} S,
\end{aligned}
$$

where the time derivatives are expressed in non-rotating axes and the integrand in the last volume integral has been linearized in the sense defined above, so that $\left(\tilde{\boldsymbol{\omega}}_{0}+\tilde{\boldsymbol{\omega}}_{B}\right)_{0}$ does not depend on $\boldsymbol{S}_{0}$ and $\boldsymbol{\omega}_{0}$. Now the interaction of $\boldsymbol{S}_{0}$ with the body rotation $\boldsymbol{\Omega}$ only contributes to the torque. Moreover, most contributions to the torque related to the ambient strain rate and vorticity in (3.14) vanish when the body does not rotate. More precisely, the only term due to the inhomogeneity of the undisturbed flow that still influences the torque when $\boldsymbol{\Omega}=\mathbf{0}$ is that associated with the possible unsteadiness of $\boldsymbol{S}_{0}$.

In (3.14) the ambient strain rate and vorticity contribute to the Lagrangian acceleration $\mathrm{D}_{0} \boldsymbol{U}_{0} / \mathrm{D} t$, whose direction is generally not aligned with that of the body's relative velocity $\tilde{\boldsymbol{V}}$. In addition, the ambient strain rate still influences the hydrodynamic force through the term $\left[\boldsymbol{A} \cdot \boldsymbol{S}_{0}-\boldsymbol{S}_{0} \cdot \boldsymbol{A}\right] \cdot \tilde{\boldsymbol{V}}$, which is non-zero only when the principal axes of $\boldsymbol{S}_{0}$ are not aligned with those of the body. In this case, this term is orthogonal to $\tilde{V}$ and hence contributes to the lift force. The pair of terms $\frac{1}{2} \boldsymbol{\omega}_{0} \times(\boldsymbol{A} \cdot \tilde{\boldsymbol{V}})-\frac{1}{2} \boldsymbol{A} \cdot\left(\tilde{\boldsymbol{V}} \times \boldsymbol{\omega}_{0}\right)$ also contributes to that component of the force. Moreover, these two contributions are supplemented by the term $\boldsymbol{A} \cdot\left[\boldsymbol{\omega}_{0} \times(\boldsymbol{A} \cdot \tilde{\boldsymbol{V}})\right] / \mathscr{V}_{B}$ resulting from (F7), which is quadratic with respect to the translational added-mass tensor. For a point-symmetric body such as an infinitely long circular cylinder or a sphere, $\boldsymbol{D}=\boldsymbol{E}_{R}=\boldsymbol{K}=\mathbf{0}$ and $\boldsymbol{A}=\mathscr{A} \boldsymbol{I}$, where $\mathscr{A} / \mathscr{V}_{B}$ is the usual added-mass coefficient. Hence the closed-form contributions to the torque vanish identically, whereas those to the force reduce to $\left(\mathscr{V}_{B}+\mathscr{A}\right) \mathrm{D}_{0} \boldsymbol{U}_{0} / \mathrm{D} t-\mathscr{A} \mathrm{d} \boldsymbol{V} / \mathrm{d} t+\mathscr{A}\left(1+\mathscr{A} / \mathscr{V}_{B}\right) \boldsymbol{\omega}_{0} \times \tilde{\boldsymbol{V}}$.

On the right-hand side of (3.14), all terms resulting from the inhomogeneity of the ambient flow are similar to those obtained by Miloh ( $\square$ ), although contributions to the torque involving $\boldsymbol{S}_{0}$ and $\boldsymbol{\omega}_{0}$ are expressed in a different way. However, the fundamental difference between the two sets of results is that Miloh's derivation assumes an inviscid flow with a negligible vorticity disturbance throughout the fluid, so that all four volume and surface integrals in (3.14) do not appear. What the present result shows is that the inviscid contributions predicted in Miloh's approach survive in the presence of viscous effects and of a non-zero vorticity disturbance throughout the flow, and that a clear splitting between these various effects is naturally achieved by the extended form of the 'reciprocal' theorem derived in this section. The result (3.14) may also be seen as the generalization to anisotropic bodies and to finite-Reynolds-number conditions of the well-known expression $\boldsymbol{F}=\left(\mathscr{V}_{B}+\mathscr{A}\right) \mathrm{D}_{0} \boldsymbol{U}_{0} / \mathrm{D} t-\mathscr{A} \mathrm{d} \boldsymbol{V} / \mathrm{d} t+\mathscr{C}_{L} \mathscr{V}_{B} \boldsymbol{\omega}_{0} \times \tilde{\boldsymbol{V}}$ derived in the inviscid 
limit by Auton et al. ( $\square$ ) in the particular case of a two-dimensional circular cylinder and a sphere moving in a weakly unsteady shear flow, respectively.

As pointed out above, the group of terms $\frac{1}{2}\left\{\omega_{0} \times(\boldsymbol{A} \cdot \tilde{\boldsymbol{V}})+\boldsymbol{A} \cdot\left(\boldsymbol{\omega}_{0} \times \tilde{\boldsymbol{V}}\right)\right\}+\boldsymbol{A} \cdot$ $\left[\boldsymbol{\omega}_{0} \times(\boldsymbol{A} \cdot \tilde{\boldsymbol{V}})\right] / \mathscr{V}_{B}$ in (3.14) contributes to the lift force. Nevertheless, owing to the Helmholtz equation, the vorticity disturbance $\tilde{\boldsymbol{\omega}}$ is generally non-zero in the presence of an ambient vorticity $\omega_{0}$, even though the flow is considered inviscid, and the arrangement of $\tilde{\boldsymbol{\omega}}$ and $\tilde{\boldsymbol{\omega}}_{B}$ around the body results in a non-zero value of the remaining volume integrals that make additional contributions to this force. Therefore, evaluating the net lift force generally requires the vorticity disturbance (and velocity) to be known throughout the flow field, as exemplified by Auton's calculation for the steady lift force on a sphere in a weak shear flow (Auton $\square$ ). It is only in particular situations that the inertial closed-form terms in (3.14) represent the entire lift force. Inviscid two-dimensional flows provide such a situation, since $\tilde{\boldsymbol{\omega}}$ and $\tilde{\boldsymbol{\omega}}_{B}$ are both zero in that case, making both volume integrals in (3.14) vanish. For a two-dimensional circular cylinder, $\mathscr{A} / \mathscr{V}_{B}=1$, so that, when the cylinder moves in an inviscid shear flow with $\omega_{0}$ parallel to its axis, the lift force is found to be $2 \mathscr{V}_{B} \omega_{0} \times \tilde{\boldsymbol{V}}$, in agreement with a classical result (Batchelor $\square$ ). In three-dimensional flows, although $\tilde{\omega}$ is zero right at the time the body is introduced into the flow, the volume integrals in (3.14) become non-zero as the flow disturbance sets in, owing to the stretching/tilting term $\omega_{0} \cdot \nabla \tilde{\boldsymbol{U}}$. Therefore it is only in the short-time limit that the above closed-form terms provide the entire lift force. Miloh $(\square)$ considered this limit for a sphere (for which $\left.\mathscr{A} / \mathscr{V}_{B}=1 / 2\right)$, in which case (3.14) predicts that the lift force is $\frac{3}{4} \mathscr{V}_{B} \omega_{0} \times \tilde{\boldsymbol{V}}$, in agreement with the asymptotic determination of Legendre \& Magnaudet $(\square)$. In contrast, in the nearly steady approximation considered by Auton et al. $(\square)$, the vorticity disturbance around the sphere is non-zero, yielding $\mathscr{C}_{L}=1 / 2$ in the above simplified form of $\boldsymbol{F}$.

Finally, if the flow domain is bounded by a plane wall, the form of the 'reciprocal' theorem suitable for a non-rotating body having one of its three symmetry planes parallel to the wall is

$$
\begin{aligned}
& \hat{\boldsymbol{I}} \cdot \boldsymbol{F}+\hat{\boldsymbol{j}} \cdot \boldsymbol{\Gamma}=\hat{\boldsymbol{I}} \cdot\left\{\frac{\mathrm{D}_{0} \boldsymbol{U}_{0}}{\mathrm{D} t} \mathscr{V}_{B}-\boldsymbol{A} \cdot\left(\frac{\mathrm{d} \boldsymbol{V}}{\mathrm{d} t}-\frac{\mathrm{D}_{0} \boldsymbol{U}_{0}}{\mathrm{D} t}\right)-(\boldsymbol{V} \cdot \nabla \boldsymbol{A}) \cdot \tilde{\boldsymbol{V}}\right. \\
& +\left[\boldsymbol{A} \cdot \boldsymbol{S}_{0}-\boldsymbol{S}_{0} \cdot \boldsymbol{A}\right] \cdot \tilde{\boldsymbol{V}}+\left(\frac{\boldsymbol{I}}{2}+\frac{\boldsymbol{A}}{\mathscr{V}_{B}}\right) \\
& \left.\times\left[\omega_{0} \times(\boldsymbol{A} \cdot \tilde{\boldsymbol{V}})\right]-\frac{1}{2} \boldsymbol{A} \cdot\left(\tilde{\boldsymbol{V}} \times \boldsymbol{\omega}_{0}\right)\right\} \\
& -\hat{\boldsymbol{j}} \cdot\left\{\tilde{\boldsymbol{V}} \times(\boldsymbol{A} \cdot \tilde{\boldsymbol{V}})+\boldsymbol{E}_{R}: \frac{\mathrm{d} \boldsymbol{S}_{0}}{\mathrm{~d} t}-\frac{1}{2}(\boldsymbol{V} \cdot \nabla \boldsymbol{D}) \cdot \boldsymbol{\omega}_{0}+\left(\boldsymbol{V} \cdot \nabla \boldsymbol{E}_{R}\right): \boldsymbol{S}_{0}\right\} \\
& -\int_{\mathscr{V}} \hat{\boldsymbol{\phi}} \boldsymbol{\omega}_{0} \cdot\left(\tilde{\boldsymbol{\omega}}+\tilde{\boldsymbol{\omega}}_{B}\right)_{0} \mathrm{~d} V+\int_{\mathscr{V}}\left[\left(\tilde{\boldsymbol{\omega}}+\tilde{\boldsymbol{\omega}}_{B}\right) \times \tilde{\boldsymbol{U}}\right]_{0} \cdot(\hat{\boldsymbol{W}}-\hat{\boldsymbol{U}}) \mathrm{d} V \\
& -\frac{1}{R e} \int_{S_{B}}\{(\hat{\boldsymbol{U}}-\hat{\boldsymbol{W}}) \times(\boldsymbol{\omega}-2 \boldsymbol{\Omega})\} \cdot \boldsymbol{n} \mathrm{d} S-\frac{2}{R e} \hat{\boldsymbol{j}} \cdot \int_{S_{B}} \boldsymbol{n} \times(\tilde{\boldsymbol{U}}-\tilde{\boldsymbol{W}}) \mathrm{d} S \\
& -\int_{S_{W}}\left\{\frac{1}{2}(\tilde{\boldsymbol{U}} \cdot \tilde{\boldsymbol{U}})_{0} \hat{\boldsymbol{W}}+\hat{\phi} \tilde{\boldsymbol{U}}_{0} \times \boldsymbol{\omega}_{0}+\frac{1}{R e} \hat{\boldsymbol{U}} \times \boldsymbol{\omega}\right\} \cdot \boldsymbol{n} \mathrm{d} S .
\end{aligned}
$$

Note that (3.15) is still valid for a rotating circular cylinder or a rotating sphere since rotation does not induce any inviscid effect for such point-symmetric bodies. In 
addition, $\boldsymbol{E}_{R}=\boldsymbol{D}=\mathbf{0}$ in that case, so that the last three terms in the expression for the torque vanish. In contrast, $\boldsymbol{A}$ is generally not spherical even for point-symmetric bodies, since the added-mass coefficients corresponding to an acceleration parallel or perpendicular to the wall may differ from each other.

\section{Some applications to high-Reynolds-number bubble dynamics}

In this section we consider three applications of the various forms of the theorem derived in $\S \S 2$ and 3 to the prediction of high-Reynolds-number bubble motion. The fact that the surface vorticity generated by the shear-free boundary condition is of $O(1)$ (once normalized by the ratio of the slip velocity $V_{0}$ over some characteristic length scale $R$ of the bubble) instead of being of $O\left(R e^{1 / 2}\right)$ when the no-slip condition holds makes viscous corrections much weaker for bubbles than for solid bodies. More precisely, the normalized tangential velocity changes by $O\left(R e^{-1 / 2}\right)$ within the boundary layer surrounding a bubble, instead of changing by $O(1)$ near a no-slip surface (Moore ए; Batchelor $\square$ ). Therefore, in the limit of very large Reynolds number, the bound vorticity $\omega_{B}$ in (2.19) (or $\tilde{\omega}_{B}$ in (3.6)) is negligibly small and the effect of the shear-free condition can be considered as merely resulting in a vortex sheet right at the bubble surface. This major difference with solid bodies makes it possible to perform asymptotic calculations in the limit $R e \rightarrow \infty$ so as to obtain consistent force and torque expressions including both inertial and leading-order viscous effects. Conversely, in situations where inviscid theory predicts a non-zero force or torque, viscous effects can only induce a small correction to that prediction, not a contribution of similar or even larger magnitude as may be the case with the no-slip condition. For these reasons it sounds interesting to examine how results derived in the previous sections may be applied to predict inertial and/or viscous loads on shear-free bubbles in some contrasting situations.

\subsection{Two spherical bubbles rising side by side in a liquid at rest}

To illustrate the ability of (2.19) to predict both drag and lift components of the force, we first consider how it applies to the well-understood situation of two identical spherical bubbles rising steadily in such a way that their line of centres stays perpendicular to their rise velocity (Legendre, Magnaudet \& Mougin $\square$ ). The dimensional distance between the two bubbles is $2 L$, so that it is convenient to define the dimensionless quantity $\kappa=R / L$, which represents the inverse of the separation from one bubble to the symmetry plane of the system. Hence this symmetry plane now stands at $\boldsymbol{r} \cdot \boldsymbol{e}_{\perp}=-1 / \kappa$, where $\boldsymbol{e}_{\perp}$ is the unit vector in the direction of the line of centres pointing away from the symmetry plane (figure $\lceil a$ ). We assume the separation $L$ to be much larger than the bubble radius $R$, i.e. we consider the limit $\kappa \ll 1$. In a first step we assume that the Reynolds number is infinite but the fluid is still viscous, so that the velocity disturbance may be considered irrotational except right at the surface of the bubbles. Corrections related to the finiteness of $R e$ will be considered later. Consequently, at leading order in $R e^{-1}$ and in $\kappa$ (i.e. in the limit $\kappa \rightarrow 0$ ), the velocity disturbance about the bubble centred at $\boldsymbol{r}=\mathbf{0}$ (say bubble $A$ ) is merely the dipole solution $\boldsymbol{U}_{0}=-\boldsymbol{V} \cdot \boldsymbol{\nabla}\left(\boldsymbol{r} / 2 r^{3}\right)$ (hence $\boldsymbol{V}$ is the rise velocity in the limit $\kappa \rightarrow 0$ ). To cancel the normal velocity created by this dipole on the symmetry plane, a dipole of similar strength must be introduced at the image point $r=-(2 / \kappa) \boldsymbol{e}_{\perp}$ (i.e. at the centre of the second bubble, say $B$ ). The velocity field induced by this image dipole is of course $\boldsymbol{U}_{0}^{*}=-\boldsymbol{V} \cdot \nabla\left(\boldsymbol{r}^{*} / 2 r^{* 3}\right)$, with $\boldsymbol{r}^{*}=\boldsymbol{r}+2 \kappa^{-1} \boldsymbol{e}_{\perp}$ and $r^{*}=\left\|\boldsymbol{r}^{*}\right\|$. Expanding $\boldsymbol{U}_{0}^{*}$ in 

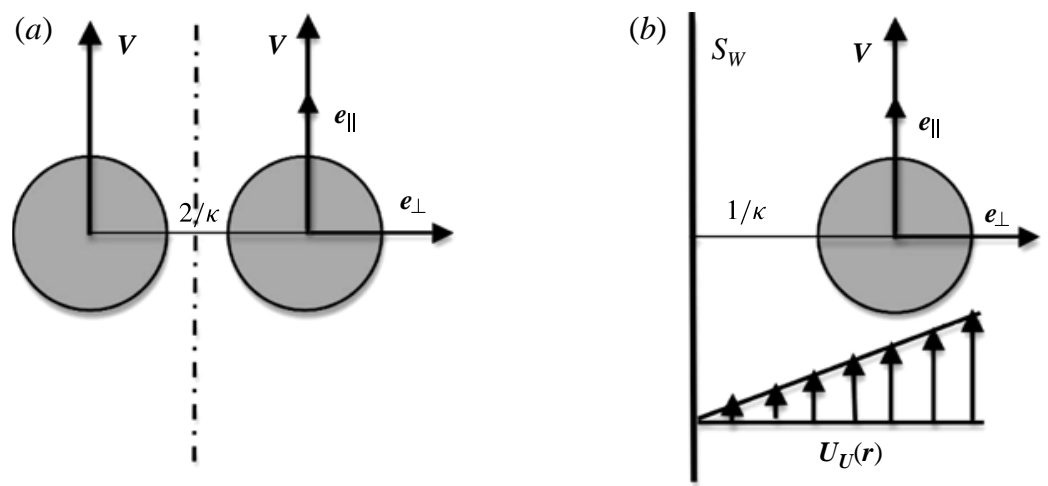

FIGURE 2. Flow configurations considered in $(a) \S 4.1$ and $(b) \S 4.3$.

the vicinity of bubble $A$ yields

$$
\boldsymbol{U}_{0}^{*}(\kappa\|\boldsymbol{r}\| \ll 1)=\boldsymbol{U}_{\infty}(\boldsymbol{r})=-\frac{\kappa^{3}}{16} \boldsymbol{V}+\frac{3 \kappa^{4}}{32}\left(\left(\boldsymbol{e}_{\perp} \cdot \boldsymbol{r}\right) \boldsymbol{V}+(\boldsymbol{V} \cdot \boldsymbol{r}) \boldsymbol{e}_{\perp}\right)+O\left(\kappa^{5}\right) .
$$

Hence bubble $A$ experiences a 'far-field' velocity made up of a uniform velocity $-\left(\kappa^{3} / 16\right) \boldsymbol{V}$ and a straining field $\left(3 \kappa^{4} / 32\right)\left(\left(\boldsymbol{e}_{\perp} \cdot \boldsymbol{r}\right) \boldsymbol{V}+(\boldsymbol{V} \cdot \boldsymbol{r}) \boldsymbol{e}_{\perp}\right)$. To satisfy the kinematic condition (2.3) at the bubble surface up to terms of $O\left(\kappa^{4}\right)$, one has to add a dipole and a quadrupole with the proper strengths. The final velocity field near bubble $A$ then reads

$$
\boldsymbol{U}^{A}(\boldsymbol{r})=\boldsymbol{U}_{\infty}(\boldsymbol{r})-\frac{1}{2}\left(1+\frac{\kappa^{3}}{16}\right) \boldsymbol{V} \cdot \nabla\left(\frac{\boldsymbol{r}}{r^{3}}\right)-\frac{\kappa^{4}}{48}\left\{\boldsymbol{e}_{\perp} \boldsymbol{V}: \nabla \nabla\left(\frac{\boldsymbol{r}}{r^{3}}\right)\right\}+O\left(\kappa^{5}\right) .
$$

The auxiliary velocity field required to apply the 'reciprocal' theorem in the $\boldsymbol{V}$ direction corresponds to the particular choice $\hat{\boldsymbol{W}}=\hat{\boldsymbol{i}}=\boldsymbol{V} /\|\boldsymbol{V}\|$, so that $\hat{\boldsymbol{U}}=\boldsymbol{U}^{A} /\|\boldsymbol{V}\|$. Since the flow is steady, the first contribution on the right-hand side of (2.19) is zero. The vorticity is zero except right on the bubble surface, which indicates that the second contribution is also zero, although we do not know yet how it goes to zero when $\operatorname{Re} \rightarrow \infty$. Moreover $\boldsymbol{V} \cdot \boldsymbol{n}=\hat{\boldsymbol{i}} \cdot \boldsymbol{n}=0$ on $S_{W}$ since the motion is parallel to the symmetry plane, so that there is no contribution from $S_{W}$. Hence only the third integral is non-zero. The corresponding contribution is more easily evaluated using the form (B 6), namely

$$
\boldsymbol{V} \cdot \boldsymbol{F}=-\frac{2\|\boldsymbol{V}\|}{\operatorname{Re}} \int_{S_{B}}\left(\boldsymbol{U}_{S}^{A}-\boldsymbol{V}_{S}\right) \cdot \nabla_{S} \boldsymbol{n} \cdot\left(\hat{\boldsymbol{U}}_{S}-\hat{\boldsymbol{V}}_{S}\right) \mathrm{d} S .
$$

In a system of spherical coordinates $(r, \theta, \phi)$ with the axis $\theta=0$ corresponding to the direction of $\boldsymbol{V}$, we have $\boldsymbol{V}_{S}=-\boldsymbol{e}_{\theta} \sin \theta$ and the curvature tensor can be written as $\nabla_{S} \boldsymbol{n}=\boldsymbol{e}_{\theta} \boldsymbol{e}_{\theta}+\boldsymbol{e}_{\phi} \boldsymbol{e}_{\phi}$. Evaluating (4.3) then yields

$$
\boldsymbol{V} \cdot \boldsymbol{F}=-\frac{12}{\operatorname{Re}} \pi\left(1+\frac{\kappa^{3}}{8}\right) \boldsymbol{V} \cdot \boldsymbol{V}+O\left(\kappa^{6}\right)
$$

Hence, provided the volume integral on the right-hand side of (2.19) decays faster than $R e^{-1}$ when $R e \rightarrow \infty$ (which we shall show later to be the case), the leading-order drag is given by (4.4), so that the dimensional drag is $\boldsymbol{F}=-12 \pi \rho \nu R V_{0}\left(1+\kappa^{3} / 8\right) \boldsymbol{V}$. 
This prediction agrees with that derived by Kok $(\square)$ at $O\left(\kappa^{3}\right)$ in the limit $\kappa \ll 1, \operatorname{Re} \rightarrow \infty$ using the 'dissipation' theorem. When $\kappa \rightarrow 0$, the above result obviously corresponds to Levich's prediction for the drag of a single spherical bubble rising with an infinitely large Reynolds number in an unbounded liquid at rest at infinity (Levich $\square$, $\square$; Batchelor $\square$ ).

To make use now of (2.18) in the direction of the line of centres, we need the auxiliary velocity field corresponding to $\hat{\boldsymbol{i}}=\boldsymbol{e}_{\perp}$. By a similar method, we find that the velocity field induced near bubble $A$ by the corresponding image dipole is

$$
\boldsymbol{U}_{\infty}(\boldsymbol{r})=\frac{\kappa^{3}}{8} \boldsymbol{e}_{\perp}+\frac{3 \kappa^{4}}{32}\left(\boldsymbol{r}-3\left(\boldsymbol{r} \cdot \boldsymbol{e}_{\perp}\right) \boldsymbol{e}_{\perp}\right)+O\left(\kappa^{5}\right) .
$$

Hence, near bubble $A$, the required auxiliary velocity field correct up to terms of $O\left(\kappa^{4}\right)$ is found to be

$$
\hat{\boldsymbol{U}}(\boldsymbol{r})=\boldsymbol{U}_{\infty}(\boldsymbol{r})-\frac{1}{2}\left(1-\frac{\kappa^{3}}{8}\right) \boldsymbol{e}_{\perp} \cdot \nabla\left(\frac{\boldsymbol{r}}{r^{3}}\right)+\frac{\kappa^{4}}{32}\left\{\boldsymbol{e}_{\perp} \boldsymbol{e}_{\perp}: \nabla \nabla\left(\frac{\boldsymbol{r}}{r^{3}}\right)\right\}+O\left(\kappa^{5}\right) .
$$

The force on the bubble can now be evaluated through (2.19) in which, still in the limit of infinite Reynolds number, only the third integral and the first part of the fourth integral can be non-zero. However, the integrand of the former is an even function of the angular position along the bubble surface and hence integrates to zero. To avoid the determination of $\boldsymbol{U}$ on $S_{W}$, the latter contribution may be evaluated using the form (2.18) together with (C 3), thanks to which one obtains

$$
\boldsymbol{F} \cdot \boldsymbol{e}_{\perp}=\int_{S_{B}}\left\{\left(\frac{1}{2} \boldsymbol{U}^{A} \cdot \boldsymbol{U}^{A}\right) \boldsymbol{e}_{\perp}-\left(\boldsymbol{U}^{A} \cdot \boldsymbol{e}_{\perp}\right) \boldsymbol{V}\right\} \cdot \boldsymbol{n} \mathrm{d} S=-\frac{3}{16} \pi \kappa^{4}+O\left(\kappa^{5}\right) .
$$

Therefore, at leading order, the dimensional transverse force is $-(3 / 16) \pi \kappa^{4} \rho R^{2} V_{0}^{2} \boldsymbol{e}_{\perp}$, which is the classical result provided by irrotational flow theory (Milne-Thomson $\square$ ). However, what (4.7) additionally shows is that this prediction is not altered by the non-zero vorticity present right at the bubble surface. We shall see below how the contribution of the volume integral in (2.19), which depends on the vorticity distribution throughout the boundary layer, influences this force component.

To complete the determination of both components of $\boldsymbol{F}$ we need to evaluate the leading-order corrections induced by the finiteness of the Reynolds number. These corrections are provided by the volume integral $\mathscr{I}=\int_{\mathscr{V}}\left\{\left(\boldsymbol{\omega}+\boldsymbol{\omega}_{B}\right) \times \boldsymbol{U}\right\} \cdot(\hat{\boldsymbol{W}}-\hat{\boldsymbol{U}}) \mathrm{d} V$ in (2.19). However, they can be more straightforwardly estimated by starting from the form (2.18) of the theorem. Then, splitting the complete velocity field about bubble $A$ into the form $\boldsymbol{U}=\boldsymbol{U}^{A}+\boldsymbol{u}$, where $\boldsymbol{u}$ is the vortical correction that vanishes outside the boundary layer and wake, and making use of (C4), we have

$$
\begin{aligned}
\mathscr{I}+\int_{S_{W}} \frac{1}{2}(\boldsymbol{U} \cdot \boldsymbol{U}) \hat{\boldsymbol{W}} \cdot \boldsymbol{n} \mathrm{d} S= & \int_{S_{B}}\left\{\frac{1}{2}(\boldsymbol{U} \cdot \boldsymbol{U}) \hat{\boldsymbol{W}}-\frac{\mathrm{D} \hat{\phi}}{\mathrm{D} t} \boldsymbol{W}\right\} \cdot \boldsymbol{n} \mathrm{d} S-\int_{\mathscr{V}}(\boldsymbol{\omega} \times \boldsymbol{U}) \cdot \hat{\boldsymbol{U}} \mathrm{d} V \\
= & \int_{S_{B}}\left\{\frac{1}{2}\left(\boldsymbol{U}^{A} \cdot \boldsymbol{U}^{A}\right) \hat{\boldsymbol{W}}-\left(\boldsymbol{U}^{A} \cdot \hat{\boldsymbol{W}}\right) \boldsymbol{W}\right\} \cdot \boldsymbol{n} \mathrm{d} S \\
& +\int_{S_{B}} \frac{1}{2}(\boldsymbol{u} \cdot \boldsymbol{u}) \hat{\boldsymbol{W}} \cdot \boldsymbol{n} \mathrm{d} S
\end{aligned}
$$




$$
\begin{aligned}
& +\int_{S_{B}}\left\{\left(\boldsymbol{u} \cdot \boldsymbol{U}^{A}\right) \hat{\boldsymbol{W}}-(\boldsymbol{u} \cdot \hat{\boldsymbol{U}}) \boldsymbol{W}\right\} \cdot \boldsymbol{n} \mathrm{d} S \\
& -\int_{\mathscr{V}}\left(\boldsymbol{\omega} \times\left(\boldsymbol{U}^{A}+\boldsymbol{u}\right)\right) \cdot \hat{\boldsymbol{U}} \mathrm{d} V .
\end{aligned}
$$

The first integral on the right-hand side is the irrotational contribution that leads to (4.7) when $\hat{\boldsymbol{W}}=\boldsymbol{e}_{\perp}$. The kinematic conditions (2.13) and (2.14) may be used to transform the second and third integrals on the right-hand side, the former becoming $-\frac{1}{2} \int_{\mathscr{V}} \nabla(\boldsymbol{u} \cdot \boldsymbol{u}) \cdot \hat{\boldsymbol{U}} \mathrm{d} V$. The entire vortical contribution in (4.8) may then be written in the form

$$
\mathscr{I}=\int_{S_{B}}\left\{\boldsymbol{u} \times\left(\hat{\boldsymbol{U}} \times \boldsymbol{U}^{A}\right)\right\} \cdot \boldsymbol{n} \mathrm{d} S-\int_{\mathscr{V}} \boldsymbol{\omega} \cdot\left(\boldsymbol{U}^{A} \times \hat{\boldsymbol{U}}\right) \mathrm{d} V-\int_{\mathscr{V}}(\boldsymbol{u} \cdot \nabla \boldsymbol{u}) \cdot \hat{\boldsymbol{U}} \mathrm{d} V .
$$

Selecting $\hat{\boldsymbol{W}}=\boldsymbol{e}_{\|}$implies that $\boldsymbol{U}^{A}=\|\boldsymbol{V}\| \hat{\boldsymbol{U}}$, which shows that the first two integrals on the right-hand side of (4.9) vanish. Up to terms of $O\left(\kappa^{3}\right)$, the boundary layer that surrounds the bubble is axisymmetric and its characteristics are similar to those determined by Moore $(\square)$ for a single bubble moving at large Reynolds number in a fluid at rest at infinity. Therefore the tangential (respectively, normal) component of $\boldsymbol{u}$ is of $O\left(R e^{-1 / 2}\right)$ (respectively, $O\left(R e^{-1}\right)$ ) within the $O\left(R e^{-1 / 2}\right)$-thick boundary layer. In the last integral of (4.9) the integrand is thus of $O\left(R e^{-1}\right)$, yielding an $O\left(R e^{-3 / 2}\right)$ contribution to the drag. Owing to the straining contribution in (4.1), the vortical velocity correction has an additional non-axisymmetric $O\left(\kappa^{4}\right)$ component. In the spherical coordinate system introduced above, this component, say $\boldsymbol{u}^{N A}(r, \theta, \phi)$, is necessarily a linear function of $\sin \phi$ and $\cos \phi$, so that $\int_{0}^{2 \pi} \boldsymbol{u}^{N A}(r, \theta, \phi) \mathrm{d} \phi=\mathbf{0}$. The last integral in (4.9) involves contributions of the form $\int_{\mathscr{Y}}\left(\boldsymbol{u}^{A} \cdot \nabla \boldsymbol{u}^{N A}+\boldsymbol{u}^{N A} \cdot \nabla \boldsymbol{u}^{A}\right) \cdot \hat{\boldsymbol{U}} \mathrm{d} V$ ( $\boldsymbol{u}^{A}$ denoting the axisymmetric part of $\boldsymbol{u}$ ) but such terms are actually zero and the first correction to the drag provided by $\boldsymbol{u}^{N A}$ is expected to be associated with the term $\int_{\mathscr{V}}\left(\boldsymbol{u}^{N A} \cdot \nabla \boldsymbol{u}^{N A}\right) \cdot \hat{U} \mathrm{~d} V$, which is of $O\left(\kappa^{8}\right)$. In other words, up to that order, the finite-Re correction to (4.4) is merely that computed by Moore ( $\square$ ), so that one has

$$
\boldsymbol{V} \cdot \boldsymbol{F}=-\frac{12}{R e} \pi\left(1+\frac{\kappa^{3}}{8}\right)\left(1-1.56 \operatorname{Re}^{-1 / 2}\right) \boldsymbol{V} \cdot \boldsymbol{V}+O\left(\kappa^{6}\right),
$$

where it must be kept in mind that the present Reynolds number is based on the bubble radius, so that the factor 1.56 in (4.10) is equivalent to the more familiar factor 2.21 in Moore's original paper. The prediction (4.10) was already obtained by Legendre et al. ( $\square$, who found that it agrees well with DNS results for $R e \geqslant 20$. Let us finally examine the contribution of $\mathscr{I}$ to the transverse force. Now $\boldsymbol{U}^{A}$ and $\hat{\boldsymbol{U}}$ are no longer collinear, so that the first two integrals on the right-hand side of (4.9) are in principle non-zero and are dominated by contributions proportional to $R e^{-1 / 2}$. However, within the boundary layer, the vorticity is dominated by the radial variations of the tangential components of $\boldsymbol{u}$, i.e. one has $\boldsymbol{\omega} \approx-\left(\partial u_{\phi} / \partial r\right) \boldsymbol{e}_{\theta}+\left(\partial u_{\theta} / \partial r\right) \boldsymbol{e}_{\phi}$. Moreover, the relative variation of the irrotational fields $\boldsymbol{U}^{A}$ and $\hat{\boldsymbol{U}}$ across the boundary layer is only of $O\left(R e^{-1 / 2}\right)$, while that of $u_{\theta}$ and $u_{\phi}$ is of $O(1)$ and both vortical velocity components vanish at the outer edge of the boundary layer. Using these remarks and the kinematic conditions (2.13) and (2.14), the leadingorder contribution to the first volume integral in (4.9) can be evaluated, yielding $\int_{\mathscr{V}} \boldsymbol{\omega} \cdot\left(\boldsymbol{U}^{A} \times \hat{\boldsymbol{U}}\right) \mathrm{d} V \approx \int_{S_{B}}\left\{\left(U_{\theta}^{A} u_{\theta}+U_{\phi}^{A} u_{\phi}\right) \hat{W}_{r}-W_{r}\left(\hat{U}_{\theta} u_{\theta}+\hat{U}_{\phi} u_{\phi}\right)\right\} \mathrm{d} S$, which is just the surface integral in (4.9); in other words, the leading contribution of $\omega$ to $\mathscr{I}$ 
is balanced by that of the bound vorticity $\omega_{B}$. Hence, the first two integrals on the right-hand side of (4.9) cancel at leading order and the first non-zero difference between them arises because of the $O\left(R e^{-1 / 2}\right)$ relative variations of $\boldsymbol{U}^{A}$ and $\hat{\boldsymbol{U}}$ across the boundary layer and of the $O\left(R e^{-1 / 2}\right)$ secondary contributions to $\omega$, yielding a net contribution proportional to $R e^{-1}$. Since the first non-axisymmetric component of $\omega$ arises at $O\left(\kappa^{4}\right)$, we conclude that the difference between the leading two integrals in (4.9) yields an $O\left(\kappa^{4} R e^{-1}\right)$ contribution to the transverse force. Indeed, a viscous correction to that force component behaving as $\kappa^{4} R e^{-1}$ was detected numerically by Legendre et al. ( $\square$ ), who observed it to be repulsive and found the corresponding prefactor to be approximately 7.5 with the present definitions.

From a theoretical point of view, evaluating this viscous contribution requires solving the non-axisymmetric boundary layer problem at $O\left(\kappa^{4}\right)$. This is obviously a significant effort that is beyond the scope of the present paper. Nevertheless, even at the present qualitative stage, the formulation (2.18)-(2.19) has the decisive advantage of providing a formal expression and an order-of-magnitude estimate of this contribution and of showing by which mechanisms it is generated. None of these conclusions could have been reached with the 'dissipation' theorem, since this viscous transverse force does not produce any work. After the present investigation was completed, it was realized that results (4.4) and (4.7) and several of the above qualitative conclusions were already obtained using a formulation close to the present one by Grotta Ragazzo \& Tabak ( $\square)$.

\subsection{Viscous force and torque on an oblate spheroidal bubble translating and rotating in a liquid at rest}

Although the flow domain is unbounded and the fluid is at rest at infinity in that case, it is of interest to use (2.19) in order to evaluate all viscous contributions that can affect the motion of oblate spheroidal bubbles in the limit of very large Reynolds numbers, as this simplified geometry is known to provide a valid first-order approximation of the actual shape of millimetric bubbles moving in low-viscosity liquids (Magnaudet \& Eames $\square$ ). The corresponding inviscid contributions provided by the Kelvin-Kirchhoff equations or equivalently by the first integral on the righthand side of (2.19) are well known and will not be discussed here (they correspond to (2.26) with $\boldsymbol{B}=\boldsymbol{C}=\mathbf{0}$ and $\left.\mathrm{d}_{W} \boldsymbol{A} / \mathrm{d} t=\mathrm{d}_{W} \boldsymbol{D} / \mathrm{d} t=\mathbf{0}\right)$.

Let us first introduce the classical oblate ellipsoidal coordinate system $(\zeta, \mu, \phi)$ such that (Lamb $\square)$

$$
\left.\begin{array}{l}
x=k \zeta \mu, \quad y=k\left(1+\zeta^{2}\right)^{1 / 2}\left(1-\mu^{2}\right)^{1 / 2} \cos \phi, \\
z=k\left(1+\zeta^{2}\right)^{1 / 2}\left(1-\mu^{2}\right)^{1 / 2} \sin \phi,
\end{array}\right\}
$$

where $k$ is a constant that determines the volume of the spheroid. For $\mu \in[-1,1]$ and $\phi \in[0,2 \pi]$, the surface corresponding to $\zeta=\zeta_{0}$ is that of an oblate ellipsoid of aspect ratio $\chi=\left(1+\zeta_{0}^{2}\right)^{1 / 2} / \zeta_{0}$ and volume $\mathscr{V}=4 \pi k^{3} \zeta_{0}\left(1+\zeta_{0}^{2}\right) / 3$. The corresponding metric factors $h_{\mu}, h_{\zeta}$ and $h_{\phi}$ are given by

$$
\left.\begin{array}{l}
h_{\mu}=k\left(\frac{\zeta^{2}+\mu^{2}}{1-\mu^{2}}\right)^{1 / 2}, \quad h_{\zeta}=k\left(\frac{\zeta^{2}+\mu^{2}}{1+\zeta^{2}}\right)^{1 / 2}, \\
h_{\phi}=k\left(1-\mu^{2}\right)^{1 / 2}\left(1+\zeta^{2}\right)^{1 / 2} .
\end{array}\right\}
$$

The surface curvature tensor $\nabla_{S} \boldsymbol{n}$ may be written in the form $\nabla_{S} \boldsymbol{n}=H_{\mu}^{n}\left(\zeta_{0}\right) \boldsymbol{e}_{\mu} \boldsymbol{e}_{\mu}+$ $H_{\phi}^{n}\left(\zeta_{0}\right) \boldsymbol{e}_{\phi} \boldsymbol{e}_{\phi}$, where $\boldsymbol{e}_{\mu}$ and $\boldsymbol{e}_{\phi}$ are the unit vectors in the meridian and azimuthal 
directions, respectively, and $H_{\mu}^{n}$ and $H_{\phi}^{n}$ are the two radii of curvature, given by

$$
\left.\begin{array}{l}
H_{\mu}^{n}=\frac{1}{h_{\mu} h_{\zeta}} \frac{\partial h_{\mu}}{\partial \zeta}=\frac{\zeta\left(1+\zeta^{2}\right)^{1 / 2}}{k\left(\zeta^{2}+\mu^{2}\right)^{3 / 2}}, \\
H_{\phi}^{n}=\frac{1}{h_{\phi} h_{\zeta}} \frac{\partial h_{\phi}}{\partial \zeta}=\frac{\zeta}{k\left(1+\zeta^{2}\right)^{1 / 2}\left(\zeta^{2}+\mu^{2}\right)^{1 / 2}} .
\end{array}\right\}
$$

We assume that the minor axis of the bubble is aligned with the unit vector $\boldsymbol{e}_{x}$, whereas the equatorial plane is parallel to the $\left(\boldsymbol{e}_{y}, \boldsymbol{e}_{z}\right)$ plane. According to Lamb $(\square)$, the velocity potential corresponding to an incident velocity $\hat{i}$ and satisfying the kinematic condition (2.13) is $\hat{\phi}_{T}=\boldsymbol{\Psi}_{T} \cdot \hat{\boldsymbol{i}}$ with

$$
\begin{aligned}
\boldsymbol{\Psi}_{T}= & k \mu \frac{1-\zeta \cot ^{-1} \zeta}{\zeta_{0}\left(1+\zeta_{0}^{2}\right)^{-1}-\cot ^{-1} \zeta_{0}} \boldsymbol{e}_{x} \\
& +k \frac{\left(1-\mu^{2}\right)^{1 / 2}\left[\zeta /\left(1+\zeta^{2}\right)^{1 / 2}-\left(1+\zeta^{2}\right)^{1 / 2} \cot ^{-1} \zeta\right]}{\left(2+\zeta_{0}^{2}\right) \zeta_{0}^{-1}\left(1+\zeta_{0}^{2}\right)^{-1}-\cot ^{-1} \zeta_{0}}\left\{\cos \phi \boldsymbol{e}_{y}+\sin \phi \boldsymbol{e}_{z}\right\} .
\end{aligned}
$$

Similarly, the velocity potential corresponding to a rotation rate $\hat{j}$ is $\hat{\phi}_{R}=\boldsymbol{\Psi}_{R} \cdot \hat{j}$ with

$$
\begin{aligned}
\boldsymbol{\Psi}_{R}= & \frac{1}{3} k^{2} \mu\left(1-\mu^{2}\right)^{1 / 2} \frac{3 \zeta\left(1+\zeta^{2}\right)^{1 / 2} \cot ^{-1} \zeta-\left(3 \zeta^{2}+2\right)\left(1+\zeta^{2}\right)^{-1 / 2}}{\left(1+2 \zeta_{0}^{2}\right) \cot ^{-1} \zeta_{0}-2 \zeta_{0}-\zeta_{0} / 3\left(1+\zeta_{0}^{2}\right)^{-1}} \\
& \times\left\{\sin \phi \boldsymbol{e}_{y}-\cos \phi \boldsymbol{e}_{z}\right\} .
\end{aligned}
$$

Again, in the limit $R e \rightarrow \infty$, the velocity field $\boldsymbol{U}$ may be approximated by the irrotational form $\boldsymbol{U}=\boldsymbol{\Psi}_{T} \cdot \boldsymbol{V}+\boldsymbol{\Psi}_{R} \cdot \boldsymbol{\Omega}$ throughout the body of the flow. Selecting $\hat{\boldsymbol{U}}=\boldsymbol{\nabla} \boldsymbol{\Psi}_{T} \cdot \hat{\boldsymbol{i}}$ and implementing the above expressions in the form (B 6) of the viscous contribution to (2.19), the viscous force acting on the bubble is found to be

$$
\boldsymbol{F}=-4 \pi k\left\{P_{\|} \boldsymbol{e}_{x} \boldsymbol{e}_{x}+P_{\perp}\left(\boldsymbol{e}_{y} \boldsymbol{e}_{y}+\boldsymbol{e}_{z} \boldsymbol{e}_{z}\right)\right\} \cdot \boldsymbol{V},
$$

with

$$
\begin{aligned}
P_{\|}\left(\zeta_{0}\right) & =\frac{\left(1-\zeta_{0}^{2}\right) \zeta_{0}^{-2} \cot ^{-1} \zeta_{0}+\zeta_{0}^{-1}}{\left(1+\zeta_{0}^{2}\right)\left\{\zeta_{0}\left(1+\zeta_{0}^{2}\right)^{-1}-\cot ^{-1} \zeta_{0}\right\}^{2}}, \\
P_{\perp}\left(\zeta_{0}\right) & =2 \frac{\zeta_{0}\left(1-\zeta_{0}^{2}\right)+\left(1+\zeta_{0}^{2}\right)^{2} \cot ^{-1} \zeta_{0}}{\left\{\left(2+\zeta_{0}^{2}\right)-\zeta_{0}\left(1+\zeta_{0}^{2}\right) \cot ^{-1} \zeta_{0}\right\}^{2}} .
\end{aligned}
$$

The expression for the drag along the short axis agrees with that of Moore $(\square)$, while that for the drag along any major axis agrees with the result derived independently by van Wijngaarden $(\square)$ and by the present author (unpublished), all of them having made use of the 'dissipation' theorem in the limit $R e \rightarrow \infty$. The normalized drag coefficients $4 k P_{\|}$and $4 k P_{\perp}$ are plotted as a function of the aspect ratio $\chi$ in figure $\Gamma$. The above expressions indicate that the viscous force is generally not aligned with the upstream velocity since $P_{\|} \neq P_{\perp}$. Actually $P_{\|}$is larger than $P_{\perp}$ and the difference increases as the bubble becomes more oblate, i.e. as $\zeta_{0} \rightarrow \infty$. Hence the angle $\Psi=\sin ^{-1}\left(\left\|\boldsymbol{F}-\left(\boldsymbol{F} \cdot \boldsymbol{e}_{x}\right) \boldsymbol{e}_{x}\right\| /\|\boldsymbol{F}\|\right)$ is smaller than the drift angle $\Theta=\sin ^{-1}\left(\left\|\boldsymbol{V}-\left(\boldsymbol{V} \cdot \boldsymbol{e}_{x}\right) \boldsymbol{e}_{x}\right\| /\|\boldsymbol{V}\|\right)$ and, the larger the oblateness, the larger the difference $\Theta-\Psi$. It is also worth noting that, given the geometrical symmetries of the surface and the linearity of the viscous term in (2.19) with respect to the velocity, no 




FIGURE 3. Evolution of the normalized drag and torque coefficients versus the bubble aspect ratio $\chi=\left(1+\zeta_{0}^{2}\right)^{1 / 2} / \zeta_{0}$ for $1 \leqslant \chi \leqslant 2.5$.

viscous contribution to the force can be induced by the rotation $\boldsymbol{\Omega}$. Hence there is no viscous Magnus-like force in the high-Re limit considered here.

As far as we are aware, no result is available in the literature for the viscous torque acting on the bubble. Selecting $\hat{\boldsymbol{U}}=\nabla \boldsymbol{\Psi}_{R} \cdot \hat{\boldsymbol{j}}$, long but straightforward calculations yield

$$
\boldsymbol{\Gamma}=-4 \pi k^{3} T_{\Omega}\left(\boldsymbol{e}_{y} \boldsymbol{e}_{y}+\boldsymbol{e}_{z} \boldsymbol{e}_{z}\right) \cdot \boldsymbol{\Omega},
$$

with

$$
T_{\Omega}\left(\zeta_{0}\right)=\frac{1}{18} Q^{2}\left(\zeta_{0}\right) A\left(\zeta_{0}\right)-\frac{1}{3} Q\left(\zeta_{0}\right) B\left(\zeta_{0}\right)+\frac{1}{2} C\left(\zeta_{0}\right)
$$

where

$$
\begin{gathered}
Q\left(\zeta_{0}\right)=\frac{3\left(1+\zeta_{0}^{2}\right) \cot ^{-1} \zeta_{0}-\left(3 \zeta_{0}^{2}+2\right) \zeta_{0}^{-1}}{\left(1+2 \zeta_{0}^{2}\right)\left(1+\zeta_{0}^{2}\right) \cot ^{-1} \zeta_{0}-2 \zeta_{0}\left(1+\zeta_{0}^{2}\right)-\zeta_{0} / 3}, \\
A\left(\zeta_{0}\right)=\left(1+2 \zeta_{0}^{2}\right)\left(1-6 \zeta_{0}^{2}\right)\left(1+\zeta_{0}^{2}\right)^{2} \cot ^{-1} \zeta_{0} \\
+\zeta_{0}\left(1+\zeta_{0}^{2}\right)\left(12 \zeta_{0}^{4}+12 \zeta_{0}^{2}+1\right)+2 \zeta_{0}^{3} / 3 \\
B\left(\zeta_{0}\right)=\left(1-2 \zeta_{0}^{2}\right)\left(1+\zeta_{0}^{2}\right)^{2} \cot ^{-1} \zeta_{0}+\zeta_{0}\left(1+2 \zeta_{0}^{2}\right)\left(1+\zeta_{0}^{2}\right)-2 \zeta_{0}^{3} / 3 \\
C\left(\zeta_{0}\right)=\left(1+\zeta_{0}^{2}\right)^{2} \cot ^{-1} \zeta_{0}-\zeta_{0}\left(\zeta_{0}^{2}+5 / 3\right)
\end{gathered}
$$

The normalized torque coefficient $4 k T_{\Omega}$ is also plotted in figure $\Gamma$ and is found to increase gradually as the bubble becomes more oblate. Again note that, owing to the linearity of the viscous contribution in (2.19) and to the geometrical symmetries of the surface, no viscous torque is generated when the bubble translates with a non-zero drift angle without rotating.

Owing to the absence of such couplings between $\boldsymbol{F}$ and $\boldsymbol{\Omega}$ on the one hand and between $\boldsymbol{\Gamma}$ and $\boldsymbol{V}$ on the other, (4.16) and (4.19) represent the complete viscous contributions to the force and torque balances that govern the bubble motion in the limit $R e \rightarrow \infty$. By adding them to the inviscid contributions provided by the first integral in (2.19), one obtains rigorously the generalized form of the Kirchhoff-Kelvin equations that includes both added-mass effects and leading-order vortical contributions related to the shear-free condition (2.4). Although (4.16)-(4.18) and (4.19)-(4.24) could have been obtained using the classical 'dissipation' theorem, the task is significantly simplified by the use of (2.19), which avoids having to 
evaluate the dissipation in the body of the fluid; this is especially valuable for the calculation of $T_{\Omega}$, for which the 'dissipation' theorem has to be written in rotating axes to make the flow stationary, which generates additional contributions in the expression of the dissipation rate.

\subsection{A two-dimensional circular bubble in a wall-bounded linear shear flow}

We finally apply the results derived for linear flows in $\S 3.2$ to a situation combining a non-zero background vorticity with the presence of a nearby wall. For that purpose we consider the case of a cylindrical bubble with a circular cross-section translating steadily along a plane wall parallel to the steady linear shear flow defined by

$$
\boldsymbol{U}_{U}(\boldsymbol{r})=\boldsymbol{U}_{0}+\alpha\left(\boldsymbol{r} \cdot \boldsymbol{e}_{\perp}\right) \boldsymbol{e}_{\|}=\left(U_{0}+\alpha \boldsymbol{r} \cdot \boldsymbol{e}_{\perp}\right) \boldsymbol{e}_{\|},
$$

where $\alpha$ is the dimensionless shear rate, $U_{0}$ is the undisturbed fluid velocity at the distance $\kappa^{-1}$ from the wall at which the bubble centre stands (with $U_{0}=\alpha \kappa^{-1}$ in order for the undisturbed flow to satisfy the no-slip condition on the wall), and $\boldsymbol{e}_{\|}$and $\boldsymbol{e}_{\perp}$ again denote the unit vectors in directions parallel and normal to the wall, respectively (figure $\lceil b$ ). In the inviscid limit, the problem reduces to that of the flow past a circular cylinder translating near a plane wall; the corresponding streamfunction was determined for arbitrary values of $\kappa$ in the form of an infinite series by Dériat ( ${ }$ ) but no evaluation of the hydrodynamic force was attempted. Still in the limit $R e \rightarrow \infty$, the vorticity disturbance $\tilde{\boldsymbol{\omega}}$ is identically zero since the base vorticity $\omega_{0}$ is not distorted by the presence of the bubble, nor by that of the wall. Since the undisturbed vorticity is $\boldsymbol{\omega}_{0}=-\alpha \boldsymbol{e}_{\|} \times \boldsymbol{e}_{\perp}$ and the undisturbed strain rate is $\boldsymbol{S}_{0}=\alpha / 2\left(\boldsymbol{e}_{\|} \boldsymbol{e}_{\perp}+\boldsymbol{e}_{\perp} \boldsymbol{e}_{\|}\right)$, the Lagrangian acceleration $\mathrm{D}_{0} \boldsymbol{U}_{0} / \mathrm{D} t$ is identically zero. Therefore (3.15) indicates that the inviscid lift force acting on the bubble is

$$
\begin{aligned}
\boldsymbol{e}_{\perp} \cdot \boldsymbol{F}=\boldsymbol{e}_{\perp} & \cdot\left\{A_{\|}\left(1+\frac{A_{\perp}}{\mathscr{V}_{B}}\right) \boldsymbol{\omega}_{0} \times \tilde{\boldsymbol{V}}\right. \\
& \left.-\int_{S_{W}}\left[\frac{1}{2}(\tilde{\boldsymbol{U}} \cdot \tilde{\boldsymbol{U}})_{0} \boldsymbol{e}_{\perp}+\boldsymbol{e}_{\perp} \cdot\left(\tilde{\boldsymbol{U}}_{0} \times \boldsymbol{\omega}_{0}\right) \boldsymbol{\Psi}_{T \perp}\right] \mathrm{d} S\right\},
\end{aligned}
$$

where $\boldsymbol{\Psi}_{T \perp}$ (respectively, $\boldsymbol{\Psi}_{T \|}$ ) is the translational potential corresponding to a unit bubble velocity in the direction perpendicular (respectively, parallel) to the wall, and similar definitions apply to the components $A_{\perp}$ and $A_{\|}$of the translational added-mass tensor. To determine $A_{\|}$and $A_{\perp}$, we need to know $\boldsymbol{\Psi}_{T \perp}$ and $\boldsymbol{\Psi}_{T \|}$ on the bubble surface. Using techniques similar to those employed in $\S 4.1$, we obtain

$$
\begin{aligned}
\boldsymbol{\Psi}_{T \|}(\kappa\|\boldsymbol{r}\| \ll 1)= & -\frac{\boldsymbol{r}}{r^{2}}-\frac{\kappa^{2}}{4}\left\{\boldsymbol{r}+\frac{\boldsymbol{r}}{r^{2}}\right\} \\
& +\frac{\kappa^{3}}{4}\left\{\left(\boldsymbol{e}_{\perp} \cdot \boldsymbol{r}\right) \boldsymbol{r}-\frac{1}{2} \boldsymbol{e}_{\perp} \cdot \nabla\left(\frac{\boldsymbol{r}}{r^{2}}\right)\right\}+O\left(\kappa^{4}\right), \\
\boldsymbol{\Psi}_{T \perp}(\kappa\|\boldsymbol{r}\| \ll 1)= & -\frac{\boldsymbol{r}}{r^{2}}-\frac{\kappa^{2}}{4}\left\{\boldsymbol{r}+\frac{\boldsymbol{r}}{r^{2}}\right\} \\
& -\frac{\kappa^{3}}{4}\left\{\frac{r^{2}}{2} \boldsymbol{e}_{\perp}-\left(\boldsymbol{e}_{\perp} \cdot \boldsymbol{r}\right) \boldsymbol{r}-\frac{1}{2} \boldsymbol{e}_{\perp} \cdot \nabla\left(\frac{\boldsymbol{r}}{r^{2}}\right)\right\}+O\left(\kappa^{4}\right) .
\end{aligned}
$$

Inserting these expressions in (2.24), it turns out that

$$
A_{\|}=A_{\perp}=\pi\left(1+\frac{\kappa^{2}}{2}\right)+O\left(\kappa^{4}\right) .
$$


This result indicates that the translational added-mass tensor is still spherical at $O\left(\kappa^{2}\right)$ in the presence of a plane wall. Actually, this property, which is specific to circular cylindrical bodies, may be shown to subsist whatever the truncation order in $\kappa$ (Korotkin $\square$ ).

We then need to determine the relative velocity field $\tilde{\boldsymbol{U}}$ on $S_{W}$ to evaluate the corresponding integral in (4.26). Combining the dipole and quadrupole required to satisfy the kinematic boundary condition at the bubble surface with their images with respect to the wall, one merely has at leading order

$$
\begin{aligned}
\tilde{\boldsymbol{U}}\left(\boldsymbol{r} \cdot \boldsymbol{e}_{\perp}=-1 / \kappa\right)= & -\tilde{V} \boldsymbol{e}_{\|} \cdot \nabla\left(\frac{\boldsymbol{r}}{r^{2}}+\frac{\boldsymbol{r}^{*}}{r^{* 2}}\right) \\
& -\frac{\alpha}{4} \boldsymbol{e}_{\|} \boldsymbol{e}_{\perp}: \nabla \nabla\left(\frac{\boldsymbol{r}}{r^{2}}-\frac{\boldsymbol{r}^{*}}{r^{* 2}}\right)+O\left(\kappa^{4}\right),
\end{aligned}
$$

where $\tilde{V}=\left(\boldsymbol{V}-\boldsymbol{U}_{0}\right) \cdot \boldsymbol{e}_{\|}$is the relative velocity of the bubble and $\boldsymbol{r}^{*}$ is the current distance measured from the centre of the image bubble as in $\S 4.1$. Obviously this velocity field does not satisfy the no-slip boundary condition on $S_{W}$. The corresponding tangential velocity is of $O\left(\kappa^{2}\right)$, inducing an $O\left(\kappa^{2} R e^{1 / 2}\right)$ extra vorticity along the wall. The associated viscous correction to the force could be evaluated by solving the corresponding boundary layer equations (see e.g. Sherwood $\square$ in the axisymmetric case). However, this task is beyond the scope of the present work and will not be attempted here, given that the resulting correction to the force is only of $O\left(\kappa^{2} R e^{-1 / 2}\right)$ and hence negligible compared to the inertial contribution in the limit $\operatorname{Re} \rightarrow \infty$.

On $S_{W}$ the translational potential $\boldsymbol{\Psi}_{T \perp}$ is also at leading order

$$
\boldsymbol{\Psi}_{T \perp}\left(\boldsymbol{r} \cdot \boldsymbol{e}_{\perp}=-1 / \kappa\right)=-\left(\frac{\boldsymbol{r}}{r^{2}}-\frac{\boldsymbol{r}^{*}}{r^{* 2}}\right)+O\left(\kappa^{4}\right) .
$$

Making use of the above expressions, the wall contribution is obtained as

$$
-\int_{S_{W}}\left[\frac{1}{2} \tilde{\boldsymbol{U}}_{0}^{2}+\boldsymbol{e}_{\perp} \cdot\left(\tilde{\boldsymbol{U}}_{0} \times \boldsymbol{\omega}_{0}\right) \boldsymbol{\Psi}_{T \perp} \cdot \boldsymbol{e}_{\perp}\right] \mathrm{d} S=\pi \kappa^{2} \alpha \tilde{V}-\frac{\pi}{2} \kappa^{3} \tilde{V}^{2}+O\left(\kappa^{4}\right) .
$$

Note that terms proportional to $\alpha^{2}$ have been neglected in (4.32) to remain consistent with the linearization procedure that led to (4.26). Introducing results (4.29) and (4.32) within (4.26), the net inviscid lift force acting on the bubble is finally found to be

$$
\boldsymbol{e}_{\perp} \cdot \boldsymbol{F}=-\pi\left(2+\frac{\kappa^{2}}{2}\right) \alpha \tilde{V}-\frac{\pi}{2} \kappa^{3} \tilde{V}^{2}+O\left(\kappa^{4}\right) .
$$

The interesting feature revealed by (4.33) is that, if the bubble lags behind the fluid, i.e. $\tilde{V}<0$, the shear-induced lift force tends to repel it from the wall, whereas the second term tends to attract it to the wall. Therefore, there is a critical position of the bubble given by $2 / \kappa^{3}+1 / 2 \kappa=-\tilde{V} / 2 \alpha$, i.e. $1 / \kappa \approx(-\tilde{V} / 4 \alpha)^{1 / 3}$ for $\kappa \ll 1$, at which the net lift force vanishes. This position is unstable, as the force becomes attractive (respectively, repulsive) when the bubble is displaced towards (respectively, away from) the wall.

In (3.15) it is easy to see that the only inertial term that could induce a force component parallel to the wall is the contribution $-\int_{S_{W}} \boldsymbol{e}_{\perp} \cdot\left(\tilde{\boldsymbol{U}}_{0} \times \boldsymbol{\omega}_{0}\right) \boldsymbol{\Psi}_{T \|} \cdot \boldsymbol{e}_{\|} \mathrm{d} S$. However, the integrand turns out to be an odd function of the tangential coordinate 
$\boldsymbol{r} \cdot \boldsymbol{e}_{\|}$and hence integrates to zero. Therefore, the drag on the bubble entirely results from viscous effects and is negligibly small compared to the inertial transverse force in the limit $R e \rightarrow \infty$. Interestingly, we notice that the usual contribution to the drag resulting from the non-zero vorticity at the bubble surface is of $O\left(R e^{-1}\right)$ whereas that provided by the wall is of $O\left(\kappa^{2} R e^{-1 / 2}\right)$. Hence, depending on whether $\kappa^{2} R e^{1 / 2}$ is smaller or larger than unity, the drag scales differently with the Reynolds number.

\section{Summary and concluding remarks}

Motivated by the limitations of the classical 'dissipation' theorem, especially the fact that it cannot predict the lift component of the force, and by the potentialities of Lorentz's reciprocal theorem in the Stokes flow regime, we have derived a general 'reciprocal' theorem capable of predicting all components of the force and torque acting on a rigid body moving in an incompressible inhomogeneous flow at arbitrary Reynolds number. The key to this approach, which extends that of Quartapelle \& Napolitano $(\square)$ and Howe $(\square)$, is the use of auxiliary velocity fields that are both incompressible and irrotational. The introduction of such auxiliary fields offers two main advantages. First, via the use of Green's second identity and the fact that the viscous stresses of such fields have zero flux, they allow the force and torque on the body to be expressed solely in terms of the velocity field and its derivatives. Second, by properly selecting the orientation of the auxiliary motion, all required components of the loads can be evaluated, irrespective of their orientation with respect to the relative motion between the body and fluid. In contrast with the primary velocity field, these auxiliary fields do not satisfy the dynamic boundary condition at the body surface. This complicates some steps of the derivation but offers the decisive advantage of making the explicit building of these fields possible in many flow configurations of interest, as they are not influenced by viscous effects and are thus much simpler than the actual velocity field.

We have first derived a version of the theorem suitable for the case of a body moving in a fluid at rest at infinity, possibly bounded by a rigid wall or a symmetry plane. The case of no-slip and shear-free boundary conditions at the body surface have both been considered; the latter yields an additional contribution to the viscous torque due to the slip between the body and fluid. The inertial contributions from added-mass, free and bound vorticity are similar to those found by Howe $(\square)$. Terms provided by the presence of the wall consist of an inertial contribution that acts in the direction normal to it whatever the orientation of the body motion, and a viscous contribution due to the vorticity of the flow disturbance at the wall.

Two forms of the theorem, (2.18) and (2.19), have actually been derived. They are equivalent as far as the vorticity distribution in the flow is compact, a property that can always be achieved from a theoretical point of view. Things may be different when one is forced to use a control volume of finite, although large, size, such as in direct numerical simulation. For this reason, the conditions that a real flow field must fulfil for the transformation from one form of the theorem to the other to be valid have been considered. In cases where these conditions are not fulfilled, only the first form of the theorem holds.

When the body moves in a non-uniform carrying flow, three new contributions arise in the most general form (3.6) of the theorem, in addition to the net force and torque exerted by the undisturbed flow on the volume of fluid occupied by the body. Two of them are inertial by nature and involve the undisturbed vorticity throughout the flow and the undisturbed velocity gradient at the body surface, respectively. A third, viscous 
contribution arises at the body surface owing to the presence of non-zero stresses in the undisturbed flow. To make all couplings between the undisturbed velocity gradients and the body translational and rotational velocities explicit and to compare the predictions of the present approach with available results, we have considered the case of a general linear carrying flow in the weakly inhomogeneous limit where the undisturbed velocity varies over distances much larger than the body size. Expanding the various contributions involving the undisturbed strain rate and vorticity allowed us to recover all the inviscid predictions of Galper \& Miloh ( $\square)$ - which themselves encompass the theoretical findings of Taylor $(\square)$ and Tollmien $(\square)-$ and of Miloh ( $)$ for irrotational and vortical undisturbed flows, respectively. Also, all available inviscid predictions for spheres and circular cylinders immersed in linear flows, such as those of Auton et al. ( $\square)$, appear as particular cases of (3.14)-(3.15).

We have finally applied the various forms of the 'reciprocal' theorem to the prediction of inertial and viscous forces and torques acting on high-Reynolds-number bubbles submitted to a shear-free condition in several flow configurations. This enabled us to recover some well-known results classically obtained thanks to the 'dissipation' theorem or to the classical inviscid theory. In addition, we have derived several new results valid in the limit $R e \rightarrow \infty$, such as the viscous torque acting on an oblate bubble rotating in a fluid at rest, the inertial lift force on a circular bubble moving near a plane wall in a linear shear flow, or the formal expression of the $O\left(R e^{-1}\right)$ viscous correction to the transverse force acting on a pair of spherical bubbles rising side by side.

Although the Reynolds number is large in all the examples we considered, it must be stressed that the present approach remains valid at low Reynolds number. More precisely, all forms of the theorems derived in the paper reduce to terms involving a $R e^{-1}$ prefactor in the limit $R e \rightarrow 0$, possibly supplemented with addedmass contributions if unsteady and viscous effects have a comparable magnitude. In the case of an unbounded flow domain and a fluid at rest at infinity, $\S 7.1$ of Howe $(\square)$ illustrates this by considering the force acting on a rigid sphere. This section shows how the theorem (which in that case is just the linear part of (2.19) with $\hat{\boldsymbol{j}}=\mathbf{0}$ and without the wall contribution) recovers three well-known results, namely the Stokes drag and the Basset-Boussinesq history force for $R e=0$, and the low - but - finite Re Oseen correction to the drag. Owing to the neglect of nonlinear terms in that limit, only contributions associated with the vorticity distribution at the body surface contribute to the viscous force in all three cases. Given this capability of the theorems derived here to deal with low-Re situations, they may be used as an alternative to Lorentz's reciprocal theorem for determining the loads on particles moving in an inhomogeneous flow in that regime.

In the presence of finite viscous effects, expressions derived in $\S \S 2$ and 3 are of direct use to obtain closed-form predictions of the loads only in situations where the vortical velocity disturbance is weak compared to the base flow, since it is only under such a condition that the vorticity distribution can be determined through an asymptotic approach. In practice, this makes the theoretical prediction of the force and torque essentially possible for clean bubbles, with possibly the presence of a distant solid wall. Nevertheless, when a solid body is involved, these theorems are still useful in the context of experimental and computational studies. Indeed, once the vorticity distribution has been experimentally or computationally determined, they provide a direct tool for evaluating the various contributions to the loads without having to know the pressure distribution at the body surface. This approach has already been found fruitful for obtaining the time-dependent force components on bodies held fixed in 
a uniform stream, either in experiments where vorticity is determined using digital particle image velocimetry (Noca, Shiels \& Jeon $\square, \square$ ), or in computations carried out with vortex methods (Chang \& Chern $\square$; Protas et al. $\square$; Pan \& Chew $\square$. There is no doubt that it may also be helpful when the base flow is inhomogeneous, and the results derived above provide the rational basis for this.

The present approach may be extended along several lines. For instance, combined with the domain perturbation technique, it can be used to determine the inertial and viscous force and torque acting on deformable bodies, as has been classically achieved in low-Reynolds-number flows (Chan \& Leal $\square$; Magnaudet, Takagi \& Legendre $\square$ ) or in the irrotational approximation (Galper \& Miloh $\square, \square$ ). These combined approaches may also be used to determine the loads on growing or condensing vapour bubbles, for which specific questions related to momentum conservation arise, especially in the late stages of the collapse process (Eames $\square$ ). Effects of density gradients such as those evidenced by Eames \& Hunt $(\square)$, Palierne ( $\square$ ) and Miloh ( $\square$ ) may also be included. In all cases, the present approach, which stems directly from the complete Navier-Stokes equations, is the proper way to derive the correct form of the generalized Kelvin-Kirchhoff equations governing the motion of a body in a viscous fluid (Mougin \& Magnaudet $\square$ ). However, once these equations are rationally established, it is only in particular situations, such as two-dimensional inviscid flows, that all contributions can be evaluated in closed form. This is because part of the inertial contribution to the loads (especially to the lift force) lies in volume integrals involving the bound and free vorticity disturbances. Since evaluating these contributions explicitly requires the determination of the vorticity disturbance throughout the flow, it remains the 'Holy Grail' of the prediction of inertial loads acting on rigid or deformable bodies in most situations of practical interest, most notably in three-dimensional geometries.

\section{Appendix A. Strain rate and vorticity at the body surface}

Let us define an orthogonal coordinate system in which $\boldsymbol{n}$ is the unit vector tangent to one of the coordinate lines. Then any velocity field $\boldsymbol{v}$ may be split in the form $\boldsymbol{v}=\boldsymbol{v}_{S}+v_{n} \boldsymbol{n}$, where $\boldsymbol{v}_{S}$ is the projection of $\boldsymbol{v}$ onto the surface locally normal to $\boldsymbol{n}$ and $v_{n}=\boldsymbol{v} \cdot \boldsymbol{n}$. Similarly, the surface gradient operator $\nabla_{S}$ may be written in the form $\boldsymbol{\nabla}=\nabla_{S}+\boldsymbol{n} \partial / \partial n$, with $\partial / \partial n=\boldsymbol{n} \cdot \boldsymbol{\nabla}$. In addition to the obvious identities $\nabla_{S}\left(\boldsymbol{n}^{2}\right)=2 \nabla_{S} \boldsymbol{n} \cdot \boldsymbol{n}=\mathbf{0}$ and $\partial\left(\boldsymbol{n}^{2}\right) / \partial n=2 \boldsymbol{n} \cdot \partial \boldsymbol{n} / \partial n=0$, the orthogonality condition $\boldsymbol{v}_{S} \cdot \boldsymbol{n}=0$ yields the relations

$$
\left.\begin{array}{l}
\nabla_{S}\left(\boldsymbol{v}_{S} \cdot \boldsymbol{n}\right)=\nabla_{S} \boldsymbol{v}_{S} \cdot \boldsymbol{n}+\nabla_{S} \boldsymbol{n} \cdot \boldsymbol{v}_{S}=\mathbf{0}, \\
\frac{\partial\left(\boldsymbol{v}_{S} \cdot \boldsymbol{n}\right)}{\partial n}=\frac{\partial \boldsymbol{v}_{S}}{\partial n} \cdot \boldsymbol{n}+\boldsymbol{v}_{S} \cdot \frac{\partial \boldsymbol{n}}{\partial n}=0 .
\end{array}\right\}
$$

Then the velocity gradient tensor takes the form

$$
\begin{aligned}
\boldsymbol{\nabla} \boldsymbol{v}= & \nabla_{S} \boldsymbol{v}_{S}+\left(\nabla_{S} v_{n}\right) \boldsymbol{n}-\left(\nabla_{S} \boldsymbol{n} \cdot \boldsymbol{v}_{S}\right) \boldsymbol{n}+v_{n}\left(\nabla_{S} \boldsymbol{n}+\boldsymbol{n} \frac{\partial \boldsymbol{n}}{\partial n}\right) \\
& +\boldsymbol{n} \frac{\partial \boldsymbol{v}_{S}}{\partial n}+\left(\frac{\partial v_{n}}{\partial n}-\boldsymbol{v}_{S} \cdot \frac{\partial \boldsymbol{n}}{\partial n}-\boldsymbol{n} \cdot \frac{\partial \boldsymbol{v}_{S}}{\partial n}\right) \boldsymbol{n} \boldsymbol{n},
\end{aligned}
$$

where $\boldsymbol{\nabla}_{S} \boldsymbol{v}_{S}$ is the $2 \times 2$ surface velocity gradient tensor, $\boldsymbol{\nabla}_{S} \boldsymbol{n}+\boldsymbol{n}(\partial \boldsymbol{n} / \partial n)+(\partial \boldsymbol{n} / \partial n) \boldsymbol{n}$ is the $3 \times 3$ symmetric curvature tensor of the coordinate system and $\nabla_{S} \boldsymbol{n}$ is the $2 \times 2$ diagonal curvature tensor of the surface locally normal to $\boldsymbol{n}$. The trace 
$\operatorname{Tr}\left(\nabla_{S} \boldsymbol{n}\right)=\nabla_{S} \cdot \boldsymbol{n}$ is the mean curvature of the corresponding surface and (A 2) implies that the incompressibility constraint can be written as

$$
\boldsymbol{\nabla}_{S} \cdot \boldsymbol{v}_{S}+v_{n} \nabla_{S} \cdot \boldsymbol{n}+\frac{\partial v_{n}}{\partial n}-\boldsymbol{v}_{S} \cdot \frac{\partial \boldsymbol{n}}{\partial n}=0 .
$$

Introducing now the strain-rate tensor $\boldsymbol{d}=1 / 2\left(\nabla \boldsymbol{v}+{ }^{\mathrm{T}} \nabla \boldsymbol{v}\right)$, we have

$$
\begin{aligned}
2 \boldsymbol{d} \cdot \boldsymbol{n}= & \frac{\partial \boldsymbol{v}_{S}}{\partial n}-\left(\boldsymbol{n} \cdot \frac{\partial \boldsymbol{v}_{S}}{\partial n}\right) \boldsymbol{n}-\nabla_{S} \boldsymbol{n} \cdot \boldsymbol{v}_{S}+\nabla_{S} v_{n} \\
& +2\left(\frac{\partial v_{n}}{\partial n}-\boldsymbol{v}_{S} \cdot \frac{\partial \boldsymbol{n}}{\partial n}\right) \boldsymbol{n}+v_{n} \frac{\partial \boldsymbol{n}}{\partial n} .
\end{aligned}
$$

Similarly, we may split the vorticity $\omega_{v}=\nabla \times \boldsymbol{v}$ in the form $\boldsymbol{\omega}_{v}=\boldsymbol{\omega}_{S}+\omega_{n} \boldsymbol{n}$, with $\omega_{n}=\boldsymbol{\omega}_{v} \cdot \boldsymbol{n}$. Then, noting that $(\boldsymbol{\nabla} \times \boldsymbol{v}) \times \boldsymbol{n}=\boldsymbol{n} \cdot \nabla \boldsymbol{v}-\nabla \boldsymbol{v} \cdot \boldsymbol{n}$, we also have

$$
\boldsymbol{\omega}_{v} \times \boldsymbol{n}=\boldsymbol{\omega}_{S} \times \boldsymbol{n}=\frac{\partial \boldsymbol{v}_{S}}{\partial n}-\left(\boldsymbol{n} \cdot \frac{\partial \boldsymbol{v}_{S}}{\partial n}\right) \boldsymbol{n}+v_{n} \frac{\partial \boldsymbol{n}}{\partial n}+\nabla_{S} \boldsymbol{n} \cdot \boldsymbol{v}_{S}-\nabla_{S} v_{n} .
$$

Combining (A 4), (A 5) and (A 3) we then obtain

$$
2 \boldsymbol{d} \cdot \boldsymbol{n}=\boldsymbol{\omega}_{v} \times \boldsymbol{n}+2\left(\nabla_{S} v_{n}-\nabla_{S} \boldsymbol{n} \cdot \boldsymbol{v}_{S}\right)-2\left(\nabla_{S} \cdot \boldsymbol{v}_{S}+v_{n} \nabla_{S} \cdot \boldsymbol{n}\right) \boldsymbol{n} .
$$

To make use of (A 6) in the derivation of the 'reciprocal' theorem, we need to transform the term $\nabla_{S} v_{n}$ to take into account a kinematic boundary condition of the form $v_{n}=\boldsymbol{w} \cdot \boldsymbol{n}$, with $\boldsymbol{w}=\boldsymbol{a}+\boldsymbol{b} \times \boldsymbol{r}, \boldsymbol{a}$ and $\boldsymbol{b}$ being two constant vectors. One may write

$$
\nabla_{S} v_{n}=\nabla_{S}(\boldsymbol{w} \cdot \boldsymbol{n})=\nabla_{S} \boldsymbol{w} \cdot \boldsymbol{n}+\nabla_{S} \boldsymbol{n} \cdot \boldsymbol{w}=\left(\nabla \boldsymbol{w}-\boldsymbol{n} \frac{\partial \boldsymbol{w}}{\partial n}\right) \cdot \boldsymbol{n}+\nabla_{S} \boldsymbol{n} \cdot \boldsymbol{w}_{S}
$$

Then, since $\nabla \boldsymbol{w} \cdot \boldsymbol{n}=\boldsymbol{n} \times \boldsymbol{b}$, it turns out that $(\partial \boldsymbol{w} / \partial n) \cdot \boldsymbol{n}=\boldsymbol{n} \cdot \boldsymbol{\nabla} \boldsymbol{w} \cdot \boldsymbol{n}=0$, so that

$$
\nabla_{S} v_{n}=\boldsymbol{n} \times \boldsymbol{b}+\nabla_{S} \boldsymbol{n} \cdot \boldsymbol{w}_{S} .
$$

Combining (A 6) and (A 8) we find that the tangential component of the surface strain rate obeys

$$
\begin{aligned}
2(\boldsymbol{d} \cdot \boldsymbol{n})_{S} & =2 \boldsymbol{n} \times(\boldsymbol{d} \times \boldsymbol{n})=\boldsymbol{\omega}_{v} \times \boldsymbol{n}+2\left(\nabla_{S} v_{n}-\nabla_{S} \boldsymbol{n} \cdot \boldsymbol{v}_{S}\right) \\
& =\boldsymbol{\omega}_{v} \times \boldsymbol{n}-2\left\{\nabla_{S} \boldsymbol{n} \cdot\left(\boldsymbol{v}_{S}-\boldsymbol{w}_{S}\right)+\boldsymbol{b} \times \boldsymbol{n}\right\} .
\end{aligned}
$$

Hence, with an irrotational velocity field one simply has

$$
2(\boldsymbol{d} \cdot \boldsymbol{n})_{S}=-2\left\{\nabla_{S} \boldsymbol{n} \cdot\left(\boldsymbol{v}_{S}-\boldsymbol{w}_{S}\right)+\boldsymbol{b} \times \boldsymbol{n}\right\},
$$

whereas in the case of a no-slip condition the tangential surface strain rate becomes

$$
2(\boldsymbol{d} \cdot \boldsymbol{n})_{S}=\left(\boldsymbol{\omega}_{v}-2 \boldsymbol{b}\right) \times \boldsymbol{n} .
$$

Finally, for a velocity field $\boldsymbol{v}$ satisfying a shear-free boundary condition, (A 9) indicates that the tangential component of the vorticity is

$$
\boldsymbol{\omega}_{S}=\boldsymbol{n} \times\left(\boldsymbol{\omega}_{v} \times \boldsymbol{n}\right)=2 \boldsymbol{n} \times\left(\nabla_{S} \boldsymbol{n} \cdot\left(\boldsymbol{v}_{S}-\boldsymbol{w}_{S}\right)\right)+2(\boldsymbol{b}-(\boldsymbol{b} \cdot \boldsymbol{n}) \boldsymbol{n}),
$$

so that

$$
\boldsymbol{n} \times\left(\boldsymbol{\omega}_{v}-2 \boldsymbol{b}\right)=-2 \nabla_{S} \boldsymbol{n} \cdot\left(\boldsymbol{v}_{S}-\boldsymbol{w}_{S}\right) .
$$

The first term on the left-hand side of (A 13) corresponds to the vorticity induced by the relative motion of the fluid with respect to the surface, whereas the second term is due to the rotation of the surface. 


\section{Appendix B. Partial transformation of (2.16)}

Following Howe $(\square)$, the contribution on the right-hand side of (2.16) involving the acceleration $\partial \boldsymbol{U} / \partial t$ may be transformed into a surface integral by writing the integrand in the form $\hat{\boldsymbol{U}} \cdot \partial \boldsymbol{U} / \partial t=\boldsymbol{\nabla} \cdot(\hat{\phi} \partial \boldsymbol{U} / \partial t)=\partial\{\boldsymbol{\nabla} \cdot(\hat{\phi} \boldsymbol{U})\} / \partial t-\nabla \cdot\{\boldsymbol{U} \partial \hat{\phi} / \partial t\}$. Then, applying Leibnitz's theorem, keeping in mind that $S_{\infty}$ and $S_{W}$ are fixed and taking advantage of the $r^{-2}$ decay of $\hat{\phi}$ for $r \rightarrow \infty$, one obtains

$$
\begin{aligned}
\int_{\mathscr{V}} \frac{\partial \boldsymbol{U}}{\partial t} \cdot \hat{\boldsymbol{U}} \mathrm{d} V & =\frac{\mathrm{d}_{w}}{\mathrm{~d} t} \int_{\mathscr{V}} \nabla \cdot(\hat{\phi} \boldsymbol{U}) \mathrm{d} V+\int_{S_{B}} \nabla \cdot(\hat{\phi} \boldsymbol{U}) \boldsymbol{W} \cdot \boldsymbol{n} \mathrm{d} S+\int_{S_{B}} \frac{\partial \hat{\phi}}{\partial t} \boldsymbol{U} \cdot \boldsymbol{n} \mathrm{d} S \\
& =-\frac{\mathrm{d}_{W}}{\mathrm{~d} t} \int_{S_{B}} \hat{\phi} \boldsymbol{W} \cdot \boldsymbol{n} \mathrm{d} S+\int_{S_{B}}\left\{\frac{\partial \hat{\phi}}{\partial t}+\boldsymbol{U} \cdot \nabla \hat{\phi}\right\} \boldsymbol{W} \cdot \boldsymbol{n} \mathrm{d} S \\
& =-\frac{\mathrm{d}_{W}}{\mathrm{~d} t} \int_{S_{B}} \hat{\phi} \boldsymbol{W} \cdot \boldsymbol{n} \mathrm{d} S+\int_{S_{B}} \frac{D \hat{\phi}}{D t} \boldsymbol{W} \cdot \boldsymbol{n} \mathrm{d} S,
\end{aligned}
$$

where $\mathrm{d}_{w} / \mathrm{d} t$ denotes the time rate of change following the arbitrary motion of the control volume $\mathscr{V}$, while $\mathrm{d}_{W} / \mathrm{d} t$ is the time derivative following the motion of the body centre of mass and $\mathrm{D} / \mathrm{D} t=\partial / \partial t+\boldsymbol{U} \cdot \boldsymbol{\nabla}$ is the material derivative.

Now splitting the velocity $\boldsymbol{U}$ in the form $\boldsymbol{U}=\boldsymbol{U}_{S}+U_{n} \boldsymbol{n}$ and making use of (2.3), the first integral on the right-hand side of (2.16) may be written as

$$
\int_{S_{B}} \boldsymbol{U} \cdot(\hat{\boldsymbol{\tau}} \cdot \boldsymbol{n}) \mathrm{d} S=\int_{S_{B}} \boldsymbol{U}_{S} \cdot(\hat{\boldsymbol{\tau}} \cdot \boldsymbol{n})_{S} \mathrm{~d} S+\int_{S_{B}}(\boldsymbol{W} \cdot \boldsymbol{n}) \boldsymbol{n} \cdot(\hat{\boldsymbol{\tau}} \cdot \boldsymbol{n}) \mathrm{d} S .
$$

To transform the second integral on the right-hand side of (B 2), we form the curl of $\hat{\boldsymbol{U}} \times \boldsymbol{W}-\hat{\phi} \boldsymbol{\Omega}$, make use of the Stokes theorem and invoke the irrotationality condition (2.11). A little algebra then yields

$$
\mathbf{0}=2 \int_{S_{B}}\{\boldsymbol{\nabla} \times(\hat{\boldsymbol{U}} \times \boldsymbol{W}-\hat{\phi} \boldsymbol{\Omega})\} \cdot \boldsymbol{n} \mathrm{d} S=\int_{S_{B}} \boldsymbol{W} \cdot(\hat{\boldsymbol{\tau}} \cdot \boldsymbol{n}) \mathrm{d} S .
$$

We note in passing that (B 3) shows that the contribution $R^{-1} \int_{S_{B}} \boldsymbol{U} \cdot(\hat{\boldsymbol{\tau}} \cdot \boldsymbol{n}) \mathrm{d} S$ in (2.16) vanishes when the no-slip condition $\boldsymbol{U}=\boldsymbol{W}$ applies. From (B 3) we deduce the identity

$$
\int_{S_{B}}(\boldsymbol{W} \cdot \boldsymbol{n}) \boldsymbol{n} \cdot(\hat{\boldsymbol{\tau}} \cdot \boldsymbol{n}) \mathrm{d} S=-\int_{S_{B}} \boldsymbol{W}_{S} \cdot(\hat{\boldsymbol{\tau}} \cdot \boldsymbol{n})_{S} \mathrm{~d} S .
$$

With (B 4) at hand, (B 2) may be rewritten in the simpler form

$$
\int_{S_{B}} \boldsymbol{U} \cdot(\hat{\boldsymbol{\tau}} \cdot \boldsymbol{n}) \mathrm{d} S=\int_{S_{B}}\left(\boldsymbol{U}_{S}-\boldsymbol{W}_{S}\right) \cdot(\hat{\boldsymbol{\tau}} \cdot \boldsymbol{n})_{S} \mathrm{~d} S .
$$

The right-hand side of (B 5) does not vanish on a bubble surface at which the shearfree condition applies since the tangential component of the auxiliary traction, $(\hat{\boldsymbol{\tau}} \cdot \boldsymbol{n})_{S}$, does not obey any specific boundary condition on $S_{B}$. As shown in appendix A, this quantity may be transformed by exploiting the irrotational nature of $\hat{\boldsymbol{U}}$. Using (A 10) one then obtains

$$
\int_{S_{B}} \boldsymbol{U} \cdot(\hat{\boldsymbol{\tau}} \cdot \boldsymbol{n}) \mathrm{d} S=-2 \int_{S_{B}}\left(\boldsymbol{U}_{S}-\boldsymbol{W}_{S}\right) \cdot\left\{\nabla_{S} \boldsymbol{n} \cdot\left(\hat{\boldsymbol{U}}_{S}-\hat{\boldsymbol{W}}_{S}\right)+\hat{\boldsymbol{j}} \times \boldsymbol{n}\right\} \mathrm{d} S,
$$


where $\nabla_{S} \boldsymbol{n}$ is the curvature tensor of the surface. The integrand may be recast in terms of vorticity using (A 13) thanks to which (B 6) takes the form

$$
\begin{aligned}
\int_{S_{B}} \boldsymbol{U} \cdot(\hat{\boldsymbol{\tau}} \cdot \boldsymbol{n}) \mathrm{d} S= & \int_{S_{B}}\{(\boldsymbol{\omega}-2 \boldsymbol{\Omega}) \times(\hat{\boldsymbol{U}}-\hat{\boldsymbol{W}})\} \cdot \boldsymbol{n} \mathrm{d} S \\
& -2 \hat{\boldsymbol{j}} \cdot \int_{S_{B}} \boldsymbol{n} \times(\boldsymbol{U}-\boldsymbol{W}) \mathrm{d} S .
\end{aligned}
$$

The first term on the right-hand side of (B 7) represents the effect of the relative vorticity induced by the shear-free condition while the second is an additional contribution to the torque associated with the vortex sheet of strength $\boldsymbol{n} \times(\boldsymbol{U}-\boldsymbol{W})$ that exists at the bubble surface owing to the non-zero relative slip of the outer fluid. Note that the contribution of the solid-body rotation $2 \Omega$ to the force is actually zero because the part of $\hat{\boldsymbol{U}}-\hat{\boldsymbol{W}}$ associated with the translational velocity $\hat{\boldsymbol{i}}$ may be rewritten in the form $\nabla(\hat{\phi}-\hat{\boldsymbol{i}} \cdot \boldsymbol{r})$, so that $\int_{S_{B}}\{(\hat{\boldsymbol{U}}-\hat{\boldsymbol{W}}) \times \boldsymbol{\Omega}\} \cdot \boldsymbol{n} \mathrm{d} S=\int_{S_{B}} \nabla \times\{(\hat{\boldsymbol{\phi}}-\hat{\boldsymbol{i}} \cdot \boldsymbol{r}) \boldsymbol{\Omega}\} \cdot \boldsymbol{n} \mathrm{d} S$, which is zero by virtue of the Stokes theorem.

If the body rather obeys the no-slip condition (2.5), the contribution (B 2) vanishes but the last term in (2.17) is then non-zero. The surface tangential stress involved in (2.17) may again be recast in terms of the local relative vorticity thanks to (A 11), so that the corresponding contribution becomes

$$
\int_{S_{B}}(\hat{\boldsymbol{U}}-\hat{\boldsymbol{W}})_{S} \cdot(\boldsymbol{\tau} \cdot \boldsymbol{n})_{S} \mathrm{~d} S=\int_{S_{B}}\{(\hat{\boldsymbol{U}}-\hat{\boldsymbol{W}}) \times(\boldsymbol{\omega}-2 \boldsymbol{\Omega})\} \cdot \boldsymbol{n} \mathrm{d} S .
$$

Comparison of (B 8) with (B 7) indicates that the latter reduces to the former in the case of a no-slip condition $(\boldsymbol{U}=\boldsymbol{W})$, so that (B 7) represents the general form of the contribution induced by the non-zero surface vorticity whatever the specific dynamic boundary condition at the body surface. For bubbles, evaluation of (B 6) is however more straightforward since only velocities are involved.

\section{Appendix C. Partial transformation of (2.18)}

Since $\hat{\boldsymbol{U}} \cdot \partial \boldsymbol{U} / \partial t=\boldsymbol{\nabla} \cdot(\hat{\phi} \partial \boldsymbol{U} / \partial t)$, (B 1) may be written in the form

$$
\int_{S_{B}} \frac{\mathrm{D} \hat{\phi}}{\mathrm{D} t} \boldsymbol{W} \cdot \boldsymbol{n} \mathrm{d} S=\frac{\mathrm{d}_{w}}{\mathrm{~d} t} \int_{S_{B}} \hat{\boldsymbol{\phi}} \boldsymbol{U} \cdot \boldsymbol{n} \mathrm{d} S-\int_{S_{B}} \hat{\boldsymbol{\phi}} \frac{\partial \boldsymbol{U}}{\partial t} \cdot \boldsymbol{n} \mathrm{d} S .
$$

If the flow is irrotational, we can set $\boldsymbol{U}=\nabla \Phi$, and make use of the kinematic conditions (2.6) and (2.13) and of the fact that $\boldsymbol{\Phi}$ and $\hat{\boldsymbol{\phi}}$ both satisfy Laplace's equation. Hence (C1) may be expanded as

$$
\begin{aligned}
\int_{S_{B}} \frac{\mathrm{D} \hat{\phi}}{\mathrm{D} t} \boldsymbol{W} \cdot \boldsymbol{n} \mathrm{d} S= & \frac{\mathrm{d}_{w}}{\mathrm{~d} t} \int_{S_{B}}\{\hat{\phi} \nabla \Phi-\Phi(\nabla \hat{\boldsymbol{\phi}}-\hat{\boldsymbol{W}})\} \cdot \boldsymbol{n} \mathrm{d} S \\
& -\int_{S_{B}}\left\{\hat{\phi} \frac{\partial \nabla \Phi}{\partial t}-\frac{\partial \Phi}{\partial t}(\nabla \hat{\phi}-\hat{\boldsymbol{W}})\right\} \cdot \boldsymbol{n} \mathrm{d} S \\
= & -\frac{\mathrm{d}_{w}}{\mathrm{~d} t} \int_{\mathscr{V}} \nabla \cdot(\Phi \hat{\boldsymbol{W}}) \mathrm{d} V+\int_{\mathscr{V}} \nabla \cdot\left(\frac{\partial \Phi}{\partial t} \hat{\boldsymbol{W}}\right) \mathrm{d} V \\
& -\frac{\partial}{\partial t} \int_{S_{W} \cup S_{\infty}} \Phi \hat{\boldsymbol{W}} \cdot \boldsymbol{n} \mathrm{d} S+\int_{S_{W} \cup S_{\infty}} \frac{\partial \Phi}{\partial t} \hat{\boldsymbol{W}} \cdot \boldsymbol{n} \mathrm{d} S,
\end{aligned}
$$


where $\boldsymbol{n}$ is directed into the fluid on $S_{B}, S_{W}$ and $S_{\infty}$. Since $S_{W}$ and $S_{\infty}$ are fixed and $\hat{\boldsymbol{W}}$ does not depend on time, the last two integrals in (C2) cancel each other and one is left with

$$
\int_{S_{B}} \frac{\mathrm{D} \hat{\phi}}{\mathrm{D} t} \boldsymbol{W} \cdot \boldsymbol{n} \mathrm{d} S=\int_{S_{B}} \boldsymbol{\nabla} \cdot(\Phi \hat{\boldsymbol{W}}) \boldsymbol{W} \cdot \boldsymbol{n} \mathrm{d} S=\int_{S_{B}}(\boldsymbol{U} \cdot \hat{\boldsymbol{W}}) \boldsymbol{W} \cdot \boldsymbol{n} \mathrm{d} S .
$$

If the flow is not irrotational, one may still define the velocity potential $\Phi$ such that $\nabla \Phi \cdot \boldsymbol{n}=\boldsymbol{U} \cdot \boldsymbol{n}$ on $S_{B}$ and $\nabla \Phi \cdot \boldsymbol{n}=0$ on $S_{W}$ and write

$$
\begin{aligned}
\int_{S_{B}} \frac{\mathrm{D} \hat{\phi}}{\mathrm{D} t} \boldsymbol{W} \cdot \boldsymbol{n} \mathrm{d} S & =\int_{S_{B}}(\boldsymbol{U}-\nabla \Phi) \cdot \hat{\boldsymbol{U}} \boldsymbol{W} \cdot \boldsymbol{n} \mathrm{d} S+\int_{S_{B}}\left\{\frac{\partial \hat{\phi}}{\partial t}+\nabla \Phi \cdot \hat{\boldsymbol{U}}\right\} \boldsymbol{W} \cdot \boldsymbol{n} \mathrm{d} S \\
& =\int_{S_{B}}\{\nabla \Phi \cdot \hat{\boldsymbol{W}}+(\boldsymbol{U}-\nabla \Phi) \cdot \hat{\boldsymbol{U}}\} \boldsymbol{W} \cdot \boldsymbol{n} \mathrm{d} S .
\end{aligned}
$$

At this point one can introduce the bound vorticity $\boldsymbol{\omega}_{B}=\boldsymbol{n} \times(\boldsymbol{U}-\nabla \Phi) \delta_{B}$, where $\delta_{B}$ denotes the Dirac function, which is zero everywhere except on $S_{B}$ and satisfies $\int_{V} \delta_{B} \mathrm{~d} V=\int_{S_{B}} \mathrm{~d} S$. Then $\boldsymbol{\omega}_{B} \times \boldsymbol{U}=(\boldsymbol{U}-\nabla \Phi) \boldsymbol{U} \cdot \boldsymbol{n} \delta_{B}$ and $(\mathrm{C} 4)$ becomes

$$
\int_{S_{B}} \frac{D \hat{\phi}}{D t} \boldsymbol{W} \cdot \boldsymbol{n} \mathrm{d} S=\int_{S_{B}}(\nabla \Phi \cdot \hat{\boldsymbol{W}}) \boldsymbol{W} \cdot \boldsymbol{n} \mathrm{d} S+\int_{V}\left(\boldsymbol{\omega}_{B} \times \boldsymbol{U}\right) \cdot \hat{\boldsymbol{U}} \mathrm{d} V .
$$

Note that the terminology used here differs somewhat from that employed in Howe $(\square)$ where $\omega_{B}$ as defined above is called the 'excess bound vorticity'.

We may also derive the following relation

$$
\begin{aligned}
\int_{S_{B} \cup S_{W} \cup S_{\infty}}(\boldsymbol{U} \cdot \hat{\boldsymbol{W}}) \boldsymbol{U} \cdot \boldsymbol{n} \mathrm{d} S & =-\int_{V} \boldsymbol{\nabla} \cdot((\hat{\boldsymbol{W}} \cdot \boldsymbol{U}) \boldsymbol{U}) \mathrm{d} V \\
& =-\int_{\mathscr{V}} \hat{\boldsymbol{W}} \cdot \nabla\left(\frac{\boldsymbol{U} \cdot \boldsymbol{U}}{2}\right) \mathrm{d} V-\int_{\mathscr{V}} \hat{\boldsymbol{W}} \cdot(\boldsymbol{\omega} \times \boldsymbol{U}) \mathrm{d} V \\
& =-\int_{\mathscr{V}} \nabla \cdot\left(\hat{\boldsymbol{W}} \frac{\boldsymbol{U} \cdot \boldsymbol{U}}{2}\right) \mathrm{d} V-\int_{\mathscr{V}} \hat{\boldsymbol{W}} \cdot(\boldsymbol{\omega} \times \boldsymbol{U}) \mathrm{d} V,(\mathrm{C} 6)
\end{aligned}
$$

which, owing to (2.6), yields

$$
\begin{aligned}
\int_{S_{B}}(\boldsymbol{U} \cdot \hat{\boldsymbol{W}}) \boldsymbol{W} \cdot \boldsymbol{n} \mathrm{d} S= & \frac{1}{2} \int_{S_{B} \cup S_{W}}(\boldsymbol{U} \cdot \boldsymbol{U}) \hat{\boldsymbol{W}} \cdot \boldsymbol{n} \mathrm{d} S-\int_{\mathscr{V}} \hat{\boldsymbol{W}} \cdot(\boldsymbol{\omega} \times \boldsymbol{U}) \mathrm{d} V \\
& -\int_{S_{\infty}}\left\{(\boldsymbol{U} \cdot \hat{\boldsymbol{W}}) \boldsymbol{U}-\frac{1}{2}(\boldsymbol{U} \cdot \boldsymbol{U}) \hat{\boldsymbol{W}}\right\} \cdot \boldsymbol{n} \mathrm{d} S .
\end{aligned}
$$

The behaviour of the integral over $S_{\infty}$ depends critically on the decay of the velocity disturbance at large distance from the body. Axisymmetric wakes, as well as trailing vortices, have cross-sectional areas growing like $r$ (Batchelor $\square$, $\square$ ). Hence for the contribution of the outer surface to the force to be negligibly small when $r \rightarrow \infty$, it is required that $\boldsymbol{U}$ decays faster than $r^{-1 / 2}$ in the wake. The situation regarding the torque is more subtle. Clearly, a sufficient condition for the integral over $S_{\infty}$ to decay to zero as the surface recedes to infinity is that $\boldsymbol{U}$ decays faster than $r^{-1}$. However, some important situations may require a less stringent condition. For instance, if the body centre of mass moves in a straight line, $S_{\infty}$ may be chosen as a sphere (or a part of a sphere if a wall is present) within which the body moves along one of 
the diameters, which implies that $\boldsymbol{r} \times \boldsymbol{n}=\mathbf{0}$ everywhere on $S_{\infty}$, so that the normal component of the term $\hat{j} \times \boldsymbol{r}$ in $\hat{\boldsymbol{W}}$ is uniformly zero on the outer surface. Moreover $\boldsymbol{U} \cdot \hat{\boldsymbol{W}}=\hat{\boldsymbol{j}} \cdot(\boldsymbol{r} \times \boldsymbol{U})$, so that the contribution $\int_{S_{\infty}}(\boldsymbol{U} \cdot \hat{\boldsymbol{W}}) \boldsymbol{U} \cdot \boldsymbol{n} \mathrm{d} S$ can actually be written as $r \hat{\boldsymbol{j}} \cdot \int_{S_{\infty}}(\boldsymbol{n} \times \boldsymbol{U}) \boldsymbol{U} \cdot \boldsymbol{n} \mathrm{d} S$ and thus involves the product of the tangential and normal velocity components. In an axisymmetric wake, the latter decays like $r^{-1}$ while the former decays like $r^{-2}$, owing to continuity. Hence in this situation, the overall contribution decays like $r^{-1}$ and the last term in (C7) is negligibly small. It may well be that the above conditions are not naturally fulfilled in a large but finite control volume such as those used in direct numerical simulations. In such a case the contribution to the force and/or the torque provided by $S_{\infty}$ is not negligible, making (C7) useless and forcing one to stay with the form (2.18) of the theorem. From a theoretical viewpoint, the use of an extended control volume within which the vorticity distribution is compact and $\boldsymbol{U}$ then decays as $r^{-3}$ in the far field can always be invoked, making the contribution provided by $S_{\infty}$ negligible. Then we may again introduce the bound vorticity $\omega_{B}$, so as to write the left-hand side of $(\mathrm{C} 7)$ in the form

$$
\int_{S_{B}}(\boldsymbol{U} \cdot \hat{\boldsymbol{W}}) \boldsymbol{W} \cdot \boldsymbol{n} \mathrm{d} S=\int_{S_{B}}(\nabla \Phi \cdot \hat{\boldsymbol{W}}) \boldsymbol{W} \cdot \boldsymbol{n} \mathrm{d} S+\int_{\mathscr{V}}\left(\boldsymbol{\omega}_{B} \times \boldsymbol{U}\right) \cdot \hat{\boldsymbol{W}} \mathrm{d} V .
$$

Finally (C 5), (C 7) and (C 8) may be combined, yielding

$$
\begin{aligned}
\int_{S_{B}} & \left\{\frac{\mathrm{D} \hat{\phi}}{\mathrm{D} t} \boldsymbol{W}-\frac{1}{2}(\boldsymbol{U} \cdot \boldsymbol{U}) \hat{\boldsymbol{W}}\right\} \cdot \boldsymbol{n} \mathrm{d} S \\
= & \frac{1}{2} \int_{S_{W}}(\boldsymbol{U} \cdot \boldsymbol{U}) \hat{\boldsymbol{W}} \cdot \boldsymbol{n} \mathrm{d} S-\int_{\mathscr{V}}\left\{\left(\boldsymbol{\omega}_{B} \times \boldsymbol{U}\right) \cdot(\hat{\boldsymbol{W}}-\hat{\boldsymbol{U}})+(\boldsymbol{\omega} \times \boldsymbol{U}) \cdot \hat{\boldsymbol{W}}\right\} \mathrm{d} V .
\end{aligned}
$$

\section{Appendix D. Some steps required in the derivation of (3.5)}

Two structural differences between (3.4) and (2.2) have to be taken into account to obtain the counterpart of (2.18) in the case of a non-uniform undisturbed flow. One of them obviously comes from the dynamic boundary condition (2.4) or (2.5), which is still satisfied by the absolute velocity field, not by the disturbance $\tilde{U}$. The other is related to the non-uniformity of the undisturbed velocity field $\boldsymbol{U}_{U}$, which needs to be properly accounted for during the transformation of terms involving $\mathrm{D}_{U} \tilde{\boldsymbol{U}} / \mathrm{D} t$. The derivation of the counterpart of (2.18)-(2.19) is greatly simplified by considering a control volume $\mathscr{V}$ whose external boundary $S_{\infty}$ moves with the undisturbed velocity $\boldsymbol{U}_{U}$, the inner boundary $S_{B}$ still moving with the body velocity $\boldsymbol{W}$. Starting from the vector identity

$$
\boldsymbol{U}_{U} \cdot \nabla \tilde{\boldsymbol{U}} \cdot \hat{\boldsymbol{U}}=\nabla \cdot\left\{\boldsymbol{U}_{U} \nabla \cdot(\tilde{\boldsymbol{U}} \hat{\phi})\right\}-\nabla \cdot\left\{\tilde{\boldsymbol{U}} \nabla \cdot\left(\boldsymbol{U}_{U} \hat{\phi}\right)\right\}+\tilde{\boldsymbol{U}} \cdot \nabla \boldsymbol{U}_{U} \cdot \hat{\boldsymbol{U}},
$$

and taking into account the kinematic condition on $S_{W}$ for both $\tilde{\boldsymbol{U}}$ and $\boldsymbol{U}_{U}$, some elementary manipulations show that (B 1) becomes

$$
\begin{aligned}
\int_{\mathscr{V}} \frac{\mathrm{D}_{U} \tilde{\boldsymbol{U}}}{\mathrm{D} t} \cdot \hat{\boldsymbol{U}} \mathrm{d} V= & \frac{\mathrm{d}_{w}}{\mathrm{~d} t} \int_{\mathscr{V}} \nabla \cdot(\hat{\phi} \tilde{\boldsymbol{U}}) \mathrm{d} V+\int_{S_{B}} \frac{\mathrm{D} \hat{\phi}}{\mathrm{D} t} \tilde{\boldsymbol{W}} \cdot \boldsymbol{n} \mathrm{d} S \\
& +\int_{\mathscr{V}} \tilde{\boldsymbol{U}} \cdot \nabla \boldsymbol{U}_{U} \cdot \hat{\boldsymbol{U}} \mathrm{d} V+\int_{S_{\infty}}\left(\boldsymbol{U}_{U} \cdot \hat{\boldsymbol{U}}\right) \tilde{\boldsymbol{U}} \cdot \boldsymbol{n} \mathrm{d} S,
\end{aligned}
$$

still with $D \hat{\phi} / \mathrm{D} t=\partial \hat{\phi} / \partial t+\boldsymbol{U} \cdot \nabla \hat{\phi}=\mathrm{D}_{U} \hat{\phi} / \mathrm{D} t+\tilde{\boldsymbol{U}} \cdot \nabla \hat{\phi}$. 
It is then necessary to examine the way the contributions on $S_{\infty}$ encountered in the derivation of the generalization of (2.18) evolve at large distance from the body. For this purpose, let us assume that the undisturbed velocity $\boldsymbol{U}_{U}$ grows like $r^{m}$ in the far field (thus the undisturbed pressure $P_{\mathrm{U}}$ grows like $r^{m+1}$ ). Repeating the steps leading to (2.18) for the disturbance $\tilde{\boldsymbol{U}}$ and taking (D 2) into account, it is readily found that these contributions can be written as

$$
\begin{aligned}
& \frac{\mathrm{D}_{U}}{\mathrm{~d} t} \int_{S_{\infty}} \hat{\phi} \tilde{\boldsymbol{U}} \cdot \boldsymbol{n} \mathrm{d} S+\int_{S_{\infty}}\{\tilde{\boldsymbol{U}} \cdot \hat{\boldsymbol{\tau}} \cdot \boldsymbol{n}-\hat{\boldsymbol{U}} \cdot \tilde{\boldsymbol{\Sigma}} \cdot \boldsymbol{n}\} \mathrm{d} S \\
& \quad+\int_{S_{\infty}}\left\{\frac{1}{2}(\tilde{\boldsymbol{U}} \cdot \tilde{\boldsymbol{U}}) \hat{\boldsymbol{U}} \cdot \boldsymbol{n}-\left(\frac{\partial \hat{\phi}}{\partial t}+\boldsymbol{U}_{U} \cdot \hat{\boldsymbol{U}}\right) \tilde{\boldsymbol{U}} \cdot \boldsymbol{n}\right\} \mathrm{d} S .
\end{aligned}
$$

It turns out that a sufficient condition for the largest contributions on $S_{\infty}$ to be negligibly small is that $\|\tilde{\boldsymbol{U}}\|$ decays faster than $r^{N}$ with $N=\operatorname{Min}(0,1-m)$. If the vorticity distribution in $\mathscr{V}$ is compact, the velocity disturbance decays as $r^{-3}$ in the far field and any undisturbed flow with $m<4$ satisfies the above condition. In the opposite case, this condition is fulfilled at least by uniform and linear undisturbed flows but the constraint is actually less severe in most cases. For instance, if the main disturbance at large distance is due to a wake whose cross-section is of $O(r)$ when $r \rightarrow \infty$, the minimum decay rate $N$ must only satisfy $N=\operatorname{Min}(1,2-m)$, which is achieved at least up to quadratic carrying flows.

\section{Appendix E. Partial transformation of (3.5)}

Noting that $\hat{\boldsymbol{U}} \cdot \mathrm{D}_{U} \tilde{\boldsymbol{U}} / \mathrm{D} t=\nabla \cdot\left(\hat{\phi} \mathrm{D}_{U} \tilde{\boldsymbol{U}} / \mathrm{D} t\right)-\nabla \cdot\left(\hat{\phi} \tilde{\boldsymbol{U}} \cdot \nabla \boldsymbol{U}_{U}\right)+\tilde{\boldsymbol{U}} \cdot \nabla \boldsymbol{U}_{U} \cdot \hat{\boldsymbol{U}}$, then (D 2) leads to

$$
\begin{aligned}
\int_{S_{B}} \frac{\mathrm{D} \hat{\phi}}{\mathrm{D} t} \tilde{\boldsymbol{W}} \cdot \boldsymbol{n} \mathrm{d} S= & \frac{\mathrm{d}_{W}}{\mathrm{~d} t} \int_{S_{B}} \hat{\boldsymbol{\phi}} \tilde{\boldsymbol{U}} \cdot \boldsymbol{n} \mathrm{d} S \\
& -\int_{S_{B}} \hat{\boldsymbol{\phi}} \frac{\mathrm{D}_{U} \tilde{\boldsymbol{U}}}{D t} \cdot \boldsymbol{n} \mathrm{d} S+\int_{S_{B}} \hat{\phi} \tilde{\boldsymbol{U}} \cdot \nabla \boldsymbol{U}_{U} \cdot \boldsymbol{n} \mathrm{d} S .
\end{aligned}
$$

If the disturbance flow is irrotational, which implies that the undisturbed flow is also irrotational, we can set $\tilde{U}=\nabla \tilde{\Phi}$. The first two contributions on the right-hand side may be transformed using the same procedure as in (C2). Keeping in mind that the outer boundary of the control volume now moves with the normal velocity $\boldsymbol{U}_{U} \cdot \boldsymbol{n}$, this yields

$$
\begin{aligned}
\int_{S_{B}} \frac{\mathrm{D} \hat{\phi}}{\mathrm{D} t} \tilde{\boldsymbol{W}} \cdot \boldsymbol{n} \mathrm{d} S= & \frac{\mathrm{d}_{w}}{\mathrm{~d} t} \int_{S_{B}}\{\hat{\phi} \nabla \tilde{\Phi}-\tilde{\Phi}(\nabla \hat{\phi}-\hat{\boldsymbol{W}})\} \cdot \boldsymbol{n} \mathrm{d} S+\int_{S_{B}} \hat{\boldsymbol{\phi}} \tilde{\boldsymbol{U}} \cdot \nabla \boldsymbol{U}_{U} \cdot \boldsymbol{n} \mathrm{d} S \\
& -\int_{S_{B}}\left\{\hat{\phi} \frac{\mathrm{D}_{U} \nabla \tilde{\Phi}}{\mathrm{D} t}-\frac{\mathrm{D}_{U} \tilde{\Phi}}{\mathrm{D} t}(\nabla \hat{\boldsymbol{\phi}}-\hat{\boldsymbol{W}})\right\} \cdot \boldsymbol{n} \mathrm{d} S \\
= & -\frac{\mathrm{d}_{w}}{\mathrm{~d} t} \int_{\mathscr{V}} \nabla \cdot(\tilde{\Phi} \hat{\boldsymbol{W}}) \mathrm{d} V+\int_{\mathscr{V}} \nabla \cdot\left(\frac{\mathrm{D}_{U} \tilde{\Phi}}{\mathrm{D} t} \hat{\boldsymbol{W}}\right) \mathrm{d} V \\
& -\int_{\mathscr{V}} \hat{\boldsymbol{U}} \cdot\left(\nabla \boldsymbol{U}_{U}+{ }^{\mathrm{T}} \nabla \boldsymbol{U}_{U}\right) \cdot \tilde{\boldsymbol{U}} \mathrm{d} V \\
& -\frac{\mathrm{D}_{U}}{\mathrm{D} t} \int_{S_{W} \cup S_{\infty}} \tilde{\Phi} \hat{\boldsymbol{W}} \cdot \boldsymbol{n} \mathrm{d} S+\int_{S_{W} \cup S_{\infty}} \frac{\mathrm{D}_{U} \tilde{\Phi}}{\mathrm{D} t} \hat{\boldsymbol{W}} \cdot \boldsymbol{n} \mathrm{d} S,
\end{aligned}
$$


where use has been made of the kinematic conditions for both $\boldsymbol{U}_{U}$ and $\hat{\boldsymbol{U}}$ on $S_{W}$ and of the $O\left(r^{-2}\right)$ (respectively, $O\left(r^{-3}\right)$ ) decay of $\hat{\phi}$ (respectively, $\tilde{\boldsymbol{U}}$ ) as $r \rightarrow \infty$. It is then convenient to express the first contribution on the right-hand side in the form

$$
\frac{\mathrm{d}_{w}}{\mathrm{~d} t} \int_{\mathscr{V}} \nabla \cdot(\tilde{\Phi} \hat{\boldsymbol{W}}) \mathrm{d} V=\int_{\mathscr{V}} \frac{\mathrm{D}_{U}}{\mathrm{D} t}\{\nabla \cdot(\tilde{\Phi} \hat{\boldsymbol{W}})\} \mathrm{d} V-\int_{S_{B}} \nabla \cdot(\tilde{\Phi} \hat{\boldsymbol{W}}) \tilde{\boldsymbol{W}} \cdot \boldsymbol{n} \mathrm{d} S .
$$

Then making use of the identity

$$
\nabla \cdot\left(\frac{\mathrm{D}_{U} \tilde{\Phi}}{\mathrm{D} t} \hat{\boldsymbol{W}}\right)-\frac{\mathrm{D}_{U}}{\mathrm{D} t}\{\nabla \cdot(\tilde{\Phi} \hat{\boldsymbol{W}})\}=\nabla \cdot\left\{\left(\hat{\boldsymbol{W}} \cdot \nabla \boldsymbol{U}_{U}-\boldsymbol{U}_{U} \cdot \nabla \hat{\boldsymbol{W}}\right) \tilde{\Phi}\right\},
$$

and noting that $\boldsymbol{U}_{U} \cdot \nabla \hat{\boldsymbol{W}}=\hat{\boldsymbol{j}} \times \boldsymbol{U}_{U}$ and that the undisturbed flow deforms the outer surface of $\mathscr{V}$ according to $\mathrm{D}_{U}(\boldsymbol{n} \mathrm{d} S) / \mathrm{D} t=-\boldsymbol{\nabla} \boldsymbol{U}_{U} \cdot \boldsymbol{n} \mathrm{d} S$ (Batchelor $\square$ ), one obtains

$$
\begin{aligned}
\int_{S_{B}} \frac{\mathrm{D} \hat{\phi}}{\mathrm{D} t} \tilde{\boldsymbol{W}} \cdot \boldsymbol{n} \mathrm{d} S= & \int_{S_{B}}\left\{(\tilde{\boldsymbol{U}} \cdot \hat{\boldsymbol{W}}) \tilde{\boldsymbol{W}} \cdot \boldsymbol{n}+\tilde{\Phi}\left(\hat{\boldsymbol{j}} \times \boldsymbol{U}_{U}\right) \cdot \boldsymbol{n}-\tilde{\Phi} \hat{\boldsymbol{W}} \cdot \nabla \boldsymbol{U}_{U} \cdot \boldsymbol{n}\right\} \mathrm{d} S \\
& -\int_{\mathscr{V}} \hat{\boldsymbol{U}} \cdot\left(\nabla \boldsymbol{U}_{U}+{ }^{\mathrm{T}} \nabla \boldsymbol{U}_{U}\right) \cdot \tilde{\boldsymbol{U}} \mathrm{d} V .
\end{aligned}
$$

When the flow is not irrotational, one can still define the bound vorticity $\tilde{\boldsymbol{\omega}}_{B}=$ $\boldsymbol{n} \times(\tilde{\boldsymbol{U}}-\nabla \tilde{\Phi}) \delta_{B}$. Then repeating the procedure that led to (C 4) and (C 5) yields

$$
\begin{aligned}
\int_{S_{B}} \frac{\mathrm{D} \hat{\phi}}{\mathrm{D} t} \tilde{\boldsymbol{W}} \cdot \boldsymbol{n} \mathrm{d} S= & \int_{S_{B}}\left\{(\nabla \tilde{\Phi} \cdot \hat{\boldsymbol{W}}) \tilde{\boldsymbol{W}} \cdot \boldsymbol{n}+\tilde{\Phi}\left(\hat{\boldsymbol{j}} \times \boldsymbol{U}_{U}\right) \cdot \boldsymbol{n}-\tilde{\Phi} \hat{\boldsymbol{W}} \cdot \nabla \boldsymbol{U}_{U} \cdot \boldsymbol{n}\right\} \mathrm{d} S \\
& +\int_{\mathscr{V}}\left\{\tilde{\boldsymbol{\omega}}_{B} \times \tilde{\boldsymbol{U}}-\tilde{\boldsymbol{U}} \cdot\left(\nabla \boldsymbol{U}_{U}+{ }^{\mathrm{T}} \nabla \boldsymbol{U}_{U}\right)\right\} \cdot \hat{\boldsymbol{U}} \mathrm{d} V .
\end{aligned}
$$

The result (C7) still holds with $\boldsymbol{U}$ replaced by $\tilde{\boldsymbol{U}}$ and the discussion of the previous appendix regarding the conditions under which the contributions on $S_{\infty}$ are or may be made negligible still applies to the relative velocity. Therefore, provided these conditions are fulfilled, one has

$$
\begin{aligned}
& \int_{S_{B}}(\nabla \tilde{\boldsymbol{\Phi}} \cdot \hat{\boldsymbol{W}}) \tilde{\boldsymbol{W}} \cdot \boldsymbol{n} \mathrm{d} S+\int_{\mathscr{V}}\left(\tilde{\boldsymbol{\omega}}_{B} \times \tilde{\boldsymbol{U}}\right) \cdot \hat{\boldsymbol{W}} \mathrm{d} V \\
& \quad=\frac{1}{2} \int_{S_{B} \cup S_{W}}(\tilde{\boldsymbol{U}} \cdot \tilde{\boldsymbol{U}}) \hat{\boldsymbol{W}} \cdot \boldsymbol{n} \mathrm{d} S-\int_{\mathscr{V}} \hat{\boldsymbol{W}} \cdot(\tilde{\boldsymbol{\omega}} \times \tilde{\boldsymbol{U}}) \mathrm{d} V,
\end{aligned}
$$

so that the combination of (E 6) and (E 7) yields

$$
\begin{aligned}
\int_{S_{B}} & \left\{\frac{\mathrm{D} \hat{\phi}}{\mathrm{D} t} \tilde{\boldsymbol{W}} \cdot \boldsymbol{n} \mathrm{d} S-\frac{1}{2}(\tilde{\boldsymbol{U}} \cdot \tilde{\boldsymbol{U}}) \hat{\boldsymbol{W}} \cdot \boldsymbol{n}\right\} \mathrm{d} S \\
= & \int_{S_{B}}\left\{\tilde{\Phi}\left(\hat{\boldsymbol{j}} \times \boldsymbol{U}_{U}\right) \cdot \boldsymbol{n}-\tilde{\Phi} \hat{\boldsymbol{W}} \cdot \nabla \boldsymbol{U}_{U} \cdot \boldsymbol{n}\right\} \mathrm{d} S \\
& +\frac{1}{2} \int_{S_{W}}(\tilde{\boldsymbol{U}} \cdot \tilde{\boldsymbol{U}}) \hat{\boldsymbol{W}} \cdot \boldsymbol{n} \mathrm{d} S-\int_{\mathscr{V}} \hat{\boldsymbol{U}} \cdot\left(\nabla \boldsymbol{U}_{U}+{ }^{\mathrm{T}} \nabla \boldsymbol{U}_{U}\right) \cdot \tilde{\boldsymbol{U}} \mathrm{d} V \\
& +\int_{\mathscr{V}}\left(\tilde{\boldsymbol{\omega}}_{B} \times \tilde{\boldsymbol{U}}\right) \cdot(\hat{\boldsymbol{U}}-\hat{\boldsymbol{W}}) \mathrm{d} V-\int_{\mathscr{V}}(\tilde{\boldsymbol{\omega}} \times \tilde{\boldsymbol{U}}) \cdot \hat{\boldsymbol{W}} \mathrm{d} V .
\end{aligned}
$$




\section{Appendix F. Expansion of (3.13)}

In (3.13), $\int_{S_{B}} \hat{\phi} \tilde{\boldsymbol{W}} \cdot \boldsymbol{n} \mathrm{d} S$ is a function of both $t$ and $\boldsymbol{r}_{0}$, not only because the added-mass tensors may vary as the body moves perpendicularly to the wall as in $\S 2$, but also because of the spatial variation of $\tilde{\boldsymbol{V}}$. Therefore, within the 'weakly inhomogeneous' approximation, the contributions to $\left(\mathrm{d}_{W} / \mathrm{d} t\right)\left\{\int_{S_{B}} \hat{\boldsymbol{\phi}} \tilde{\boldsymbol{W}} \cdot \boldsymbol{n} \mathrm{d} S\right\}-\left(\hat{\boldsymbol{j}} \times \boldsymbol{U}_{0}\right)$. $\int_{S_{B}} \tilde{\Phi} \boldsymbol{n} \mathrm{d} S$ associated with the translational velocity $\tilde{\boldsymbol{V}}$, relative rotation rate $\tilde{\boldsymbol{\Omega}}$ and strain rate $\boldsymbol{S}_{0}$ may respectively be expanded in the form

$$
\begin{aligned}
& \frac{\mathrm{d}_{W}}{\mathrm{~d} t} \int_{S_{B}} \hat{\phi} \tilde{\boldsymbol{V}} \cdot \boldsymbol{n} \mathrm{d} S+\left(\hat{\boldsymbol{j}} \times \boldsymbol{U}_{0}\right) \cdot(\boldsymbol{A} \cdot \tilde{\boldsymbol{V}}) \\
& =-\hat{\boldsymbol{I}} \cdot\left\{\frac{\mathrm{d}_{\Omega} \boldsymbol{A}}{\mathrm{d} t} \cdot \tilde{\boldsymbol{V}}+\boldsymbol{A} \cdot\left(\frac{\partial \tilde{\boldsymbol{V}}}{\partial t}-\boldsymbol{V} \cdot \nabla \boldsymbol{U}_{0}-\boldsymbol{\Omega} \times \tilde{\boldsymbol{V}}\right)\right\} \\
& -\hat{\boldsymbol{j}} \cdot\left\{\tilde{\boldsymbol{V}} \times(\boldsymbol{A} \cdot \tilde{\boldsymbol{V}})+\frac{\mathrm{d}_{\Omega} \boldsymbol{C}}{\mathrm{d} t} \cdot \tilde{\boldsymbol{V}}+\boldsymbol{C} \cdot\left(\frac{\partial \tilde{\boldsymbol{V}}}{\partial t}-\boldsymbol{V} \cdot \nabla \boldsymbol{U}_{0}-\boldsymbol{\Omega} \times \tilde{\boldsymbol{V}}\right)\right\} \\
& +\boldsymbol{\Omega} \times(\boldsymbol{A} \cdot \tilde{\boldsymbol{V}}+\boldsymbol{C} \cdot \tilde{\boldsymbol{V}}) \\
& =-\hat{\boldsymbol{I}} \cdot\left\{\boldsymbol{A} \cdot\left(\frac{\mathrm{d} \boldsymbol{V}}{\mathrm{d} t}-\frac{\mathrm{D}_{0} \boldsymbol{U}_{0}}{\mathrm{D} t}-\boldsymbol{\Omega} \times \tilde{\boldsymbol{V}}\right)+\boldsymbol{\Omega} \times(\boldsymbol{A} \cdot \tilde{\boldsymbol{V}})\right. \\
& \left.+(\boldsymbol{V} \cdot \boldsymbol{\nabla} \boldsymbol{A}) \cdot \tilde{\boldsymbol{V}}-\boldsymbol{A} \cdot\left(\tilde{\boldsymbol{V}} \cdot \nabla \boldsymbol{U}_{0}\right)\right\} \\
& -\hat{\boldsymbol{j}} \cdot\left\{\tilde{\boldsymbol{V}} \times(\boldsymbol{A} \cdot \tilde{\boldsymbol{V}})+\boldsymbol{C} \cdot\left(\frac{\mathrm{d} \boldsymbol{V}}{\mathrm{d} t}-\frac{\mathrm{D}_{0} \boldsymbol{U}_{0}}{\mathrm{D} t}-\boldsymbol{\Omega} \times \tilde{\boldsymbol{V}}\right)+\boldsymbol{\Omega} \times(\boldsymbol{C} \cdot \tilde{\boldsymbol{V}})\right. \\
& \left.+(\boldsymbol{V} \cdot \nabla \boldsymbol{C}) \cdot \tilde{\boldsymbol{V}}-\boldsymbol{C} \cdot\left(\tilde{\boldsymbol{V}} \cdot \nabla \boldsymbol{U}_{0}\right)\right\}, \\
& \frac{\mathrm{d}_{W}}{\mathrm{~d} t} \int_{S_{B}} \hat{\phi} \tilde{\boldsymbol{\Omega}} \cdot(\boldsymbol{r} \times \boldsymbol{n}) \mathrm{d} S+\left(\hat{\boldsymbol{j}} \times \boldsymbol{U}_{0}\right) \cdot(\boldsymbol{B} \cdot \tilde{\boldsymbol{\Omega}}) \\
& =-\hat{\boldsymbol{I}} \cdot\left\{\boldsymbol{B} \cdot\left(\frac{\mathrm{d} \boldsymbol{\Omega}}{\mathrm{d} t}-\boldsymbol{\Omega} \times \tilde{\boldsymbol{\Omega}}\right)+\boldsymbol{\Omega} \times(\boldsymbol{B} \cdot \tilde{\boldsymbol{\Omega}})+(\boldsymbol{V} \cdot \nabla \boldsymbol{B}) \cdot \tilde{\boldsymbol{\Omega}}\right\} \\
& -\hat{\boldsymbol{j}} \cdot\left\{\tilde{\boldsymbol{V}} \times(\boldsymbol{B} \cdot \tilde{\boldsymbol{\Omega}})+\boldsymbol{D} \cdot\left(\frac{\mathrm{d} \boldsymbol{\Omega}}{\mathrm{d} t}-\boldsymbol{\Omega} \times \tilde{\boldsymbol{\Omega}}\right)\right. \\
& +\boldsymbol{\Omega} \times(\boldsymbol{D} \cdot \tilde{\boldsymbol{\Omega}})+(\boldsymbol{V} \cdot \boldsymbol{\nabla D}) \cdot \tilde{\boldsymbol{\Omega}}\}, \\
& -\frac{1}{2} \frac{\mathrm{d}_{W}}{\mathrm{~d} t} \int_{S_{B}} \hat{\boldsymbol{\phi}} \boldsymbol{S}_{0}:(\boldsymbol{r n}+\boldsymbol{n r}) \mathrm{d} S+\left(\hat{\boldsymbol{j}} \times \boldsymbol{U}_{0}\right) \cdot \boldsymbol{E}_{T}: \boldsymbol{S}_{0} \\
& =-\hat{\boldsymbol{I}} \cdot\left\{\boldsymbol{E}_{T}: \frac{\mathrm{d} \boldsymbol{S}_{0}}{\mathrm{~d} t}+2\left(\boldsymbol{E}_{T} \cdot \boldsymbol{S}_{0}\right):(\boldsymbol{\Omega} \cdot \boldsymbol{\epsilon})+\boldsymbol{\Omega} \times\left(\boldsymbol{E}_{T}: \boldsymbol{S}_{0}\right)+\left(\boldsymbol{V} \cdot \nabla \boldsymbol{E}_{T}\right): \boldsymbol{S}_{0}\right\} \\
& -\hat{\boldsymbol{j}} \cdot\left\{\boldsymbol{E}_{R}: \frac{\mathrm{d} \boldsymbol{S}_{0}}{\mathrm{~d} t}+2\left(\boldsymbol{E}_{R} \cdot \boldsymbol{S}_{0}\right):(\boldsymbol{\Omega} \cdot \boldsymbol{\epsilon})+\boldsymbol{\Omega} \times\left(\boldsymbol{E}_{R}: \boldsymbol{S}_{0}\right)\right. \\
& \left.+\tilde{\boldsymbol{V}} \times\left(\boldsymbol{E}_{T}: \boldsymbol{S}_{0}\right)+\left(\boldsymbol{V} \cdot \nabla \boldsymbol{E}_{R}\right): \boldsymbol{S}_{0}\right\}
\end{aligned}
$$


where $\boldsymbol{\epsilon}$ denotes the usual third-order alternating tensor and the time rate-of-change terms $\mathrm{d} \boldsymbol{V} / \mathrm{d} t$ etc. are expressed in non-rotating axes. Again, terms involving $\boldsymbol{V} \cdot \boldsymbol{\nabla} \boldsymbol{A}$ etc. result from the spatial variation of the added-mass tensors when the body velocity $\boldsymbol{V}$ has a non-zero component perpendicular to the wall and are zero otherwise. Note that these terms do not seem to satisfy Galilean invariance at first glance. However, this is simply because the wall has been assumed to be fixed in the laboratory frame, so that the problem is not translationally invariant in the wall-normal direction in this reference frame. To be more general, the wall could have been assumed to move with a velocity $\boldsymbol{V}_{W}$ and the aforementioned terms would then involve the relative velocity $\boldsymbol{V}-\boldsymbol{V}_{W}$.

The fifth contribution on the right-hand side of (3.13) combined with the terms proportional to $\tilde{\boldsymbol{V}} \cdot \boldsymbol{\nabla} \boldsymbol{U}_{0}$ in (F1) yields

$$
\begin{aligned}
\int_{S_{B}} & \left.\tilde{\Phi}_{0} \hat{\boldsymbol{W}} \cdot \nabla \boldsymbol{U}_{0} \cdot \boldsymbol{n} \mathrm{d} S-\int_{S_{B}} \tilde{\Phi}_{0} \hat{\boldsymbol{j}} \times\left(\boldsymbol{r} \cdot \nabla \boldsymbol{U}_{0}\right)\right\} \cdot \boldsymbol{n} \mathrm{d} S \\
& +\hat{\boldsymbol{I}} \cdot \boldsymbol{A} \cdot\left(\tilde{\boldsymbol{V}} \cdot \nabla \boldsymbol{U}_{0}\right)+\hat{\boldsymbol{j}} \cdot \boldsymbol{C} \cdot\left(\tilde{\boldsymbol{V}} \cdot \nabla \boldsymbol{U}_{0}\right) \\
= & \int_{S_{B}}\left\{\boldsymbol{\Psi}_{T} \cdot(\tilde{\boldsymbol{V}} \hat{\boldsymbol{I}}-\hat{\boldsymbol{I}} \tilde{\boldsymbol{V}})+\boldsymbol{\Psi}_{R} \cdot(\boldsymbol{\Omega} \hat{\boldsymbol{I}}-\hat{\boldsymbol{j}} \tilde{\boldsymbol{V}})\right\} \cdot \nabla \boldsymbol{U}_{0} \cdot \boldsymbol{n} \mathrm{d} S \\
& \left.+\int_{S_{B}}\left\{\boldsymbol{\Psi}_{T} \cdot \tilde{\boldsymbol{V}}+\boldsymbol{\Psi}_{R} \cdot \boldsymbol{\Omega}\right\}\{\hat{\boldsymbol{j}} \times \boldsymbol{r}) \cdot\left(\nabla \boldsymbol{U}_{0} \cdot \boldsymbol{n}\right)-\left[\hat{\boldsymbol{j}} \times\left(\boldsymbol{r} \cdot \nabla \boldsymbol{U}_{0}\right)\right] \cdot \boldsymbol{n}\right\} \mathrm{d} S \\
= & \hat{\boldsymbol{I}} \cdot\left\{\left[\boldsymbol{A} \cdot \boldsymbol{S}_{0}-\boldsymbol{S}_{0} \cdot \boldsymbol{A}\right] \cdot \tilde{\boldsymbol{V}}+\frac{1}{2} \boldsymbol{\omega}_{0} \times(\boldsymbol{A} \cdot \tilde{\boldsymbol{V}})\right. \\
& \left.-\frac{1}{2} \boldsymbol{A} \cdot\left(\tilde{\boldsymbol{V}} \times \boldsymbol{\omega}_{0}\right)-\boldsymbol{S}_{0} \cdot(\boldsymbol{B} \cdot \boldsymbol{\Omega})-\frac{1}{2}(\boldsymbol{B} \cdot \boldsymbol{\Omega}) \times \boldsymbol{\omega}_{0}\right\} \\
& +\hat{\boldsymbol{j}} \cdot\left\{\boldsymbol{C} \cdot\left(\boldsymbol{S}_{0} \cdot \tilde{\boldsymbol{V}}\right)-\frac{1}{2} \boldsymbol{C} \cdot\left(\tilde{\boldsymbol{V}} \times \boldsymbol{\omega}_{0}\right)+2 \boldsymbol{\epsilon}:\left(\tilde{\boldsymbol{V}} \cdot \boldsymbol{E}_{T}\right) \cdot \boldsymbol{S}_{0}+2 \boldsymbol{\epsilon}:\left(\boldsymbol{\Omega} \cdot \boldsymbol{E}_{R}\right) \cdot \boldsymbol{S}_{0}\right. \\
& \left.+\boldsymbol{O}_{T}:\left(\boldsymbol{\omega}_{0} \tilde{\boldsymbol{V}}\right)+\boldsymbol{O}_{R}:\left(\boldsymbol{\omega}_{0} \boldsymbol{\Omega}\right)\right\}
\end{aligned}
$$

where $\boldsymbol{O}_{T}$ and $\boldsymbol{O}_{R}$ are two third-order tensors, respectively defined as

$$
\boldsymbol{O}_{T}=\frac{1}{2} \int_{S_{B}}(\boldsymbol{n} \boldsymbol{r}-\boldsymbol{r} \boldsymbol{n}) \boldsymbol{\Psi}_{T} \mathrm{~d} S, \quad \boldsymbol{O}_{R}=\frac{1}{2} \int_{S_{B}}(\boldsymbol{n} \boldsymbol{r}-\boldsymbol{r n}) \boldsymbol{\Psi}_{R} \mathrm{~d} S .
$$

In (3.13) the volume integral involving the undisturbed vorticity $\omega_{0}$ may also be successively transformed as

$$
\begin{aligned}
\int_{\mathscr{V}}\left(\boldsymbol{\omega}_{0} \times \tilde{\boldsymbol{U}}_{0}\right) \cdot \hat{\boldsymbol{U}} \mathrm{d} V= & -\int_{\mathscr{V}} \boldsymbol{\omega}_{0} \cdot \nabla \times\left(\hat{\phi} \tilde{\boldsymbol{U}}_{0}\right) \mathrm{d} V+\int_{\mathscr{V}} \hat{\boldsymbol{\phi}} \boldsymbol{\omega}_{0} \cdot \tilde{\boldsymbol{\omega}}_{0} \mathrm{~d} V \\
= & \int_{\mathscr{V}} \nabla \cdot\left(\hat{\phi} \boldsymbol{\omega}_{0} \times \tilde{\boldsymbol{U}}_{0}\right) \mathrm{d} V+\int_{\mathscr{V}} \hat{\phi} \boldsymbol{\omega}_{0} \cdot \tilde{\boldsymbol{\omega}}_{0} \mathrm{~d} V \\
= & \int_{\mathscr{V}} \hat{\phi} \boldsymbol{\omega}_{0} \cdot \tilde{\boldsymbol{\omega}}_{0} \mathrm{~d} V+\boldsymbol{\omega}_{0} \cdot \int_{S_{B} \cup S_{W}} \hat{\phi}\left(\boldsymbol{n} \times \tilde{\boldsymbol{U}}_{0}\right) \mathrm{d} S \\
= & \int_{\mathscr{V}} \hat{\phi} \boldsymbol{\omega}_{0} \cdot\left(\tilde{\boldsymbol{\omega}}+\tilde{\boldsymbol{\omega}}_{B}\right)_{0} \mathrm{~d} V \\
& +\boldsymbol{\omega}_{0} \cdot \int_{S_{B}} \hat{\phi}\left(\boldsymbol{n} \times \nabla \tilde{\Phi}_{0}\right) \mathrm{d} S+\boldsymbol{\omega}_{0} \cdot \int_{S_{W}} \hat{\phi}\left(\boldsymbol{n} \times \tilde{\boldsymbol{U}}_{0}\right) \mathrm{d} S,
\end{aligned}
$$


where the index 0 in the last volume integral indicates that $\tilde{\boldsymbol{\omega}}$ and $\tilde{\boldsymbol{\omega}}_{B}$ have been linearized and no longer depend on $\boldsymbol{S}_{0}$ and $\boldsymbol{\omega}_{0}$.

Further simplifications are possible in (F 1)-(F 6) if attention is restricted to bodies with three mutually perpendicular symmetry planes that move in an unbounded fluid domain or translate without rotating near a plane wall with one of their symmetry planes parallel to that wall. Indeed, for such body shapes and flow conditions, $\boldsymbol{B}=\boldsymbol{C}=\mathbf{0}$. Also, on the surface $S_{B}$, the translational potential $\boldsymbol{\Psi}_{T}$ is then directly related to the added-mass tensor $\boldsymbol{A}$ through $\boldsymbol{\Psi}_{T}=-\boldsymbol{A} \cdot \boldsymbol{r} / \mathscr{V}_{B}$, which immediately implies $\boldsymbol{E}_{T}=\boldsymbol{O}_{T}=\mathbf{0}$. Similarly, the rotational potential $\boldsymbol{\Psi}_{R}$ then obeys $\left.\boldsymbol{\Psi}_{R}\right|_{S_{B}}=-\boldsymbol{K}:(\boldsymbol{r} \boldsymbol{r})$ (Kochin, Kibel \& Roze $\square$ ), where the third-order tensor $\boldsymbol{K}$ is connected to the rotational added-mass tensor $\boldsymbol{D}$ and to the inertia tensor $\boldsymbol{J}=\int_{\mathscr{V}_{B}}\{(\boldsymbol{r} \cdot \boldsymbol{r}) \boldsymbol{I}-\boldsymbol{r} \boldsymbol{r}\} \mathrm{d} V$ via the relation $\boldsymbol{D}=2(\boldsymbol{K} \cdot \boldsymbol{J}): \boldsymbol{\epsilon}, \boldsymbol{\epsilon}$ denoting the usual third-order alternating tensor. [Expanding this relation in index form reveals that any non-zero component of $\boldsymbol{K}$, say $K_{i j k}$, involves the ratio of the diagonal component $D_{i i}$ of $\boldsymbol{D}$ to the difference $J_{j j}-J_{k k}$ between the moments of inertia in the two principal directions $j$ and $k$ orthogonal to $i$ (Miloh ${ }^{\prime}$ ). Since $\tilde{\Phi}_{0}=\boldsymbol{\Psi}_{T} \cdot \tilde{\boldsymbol{V}}+\boldsymbol{\Psi}_{R} \cdot \boldsymbol{\Omega}$, the tangential gradient $\boldsymbol{n} \times \nabla \tilde{\Phi}_{0}$ involved in the contribution $\omega_{0} \cdot \int_{S_{B}} \hat{\phi}\left(\boldsymbol{n} \times \nabla \tilde{\Phi}_{0}\right) \mathrm{d} S$ of (F 6) may then be expressed as $\boldsymbol{n} \times \nabla \tilde{\Phi}_{0}=-\boldsymbol{n} \times\left\{(\boldsymbol{A} \cdot \tilde{\boldsymbol{V}}) / \mathscr{V}_{B}+2 \boldsymbol{\Omega} \cdot \boldsymbol{K} \cdot \boldsymbol{r}\right\}$, so that the corresponding integral can be evaluated in closed form with respect to $\boldsymbol{A}$ and $\boldsymbol{K}$, yielding

$$
\begin{aligned}
\boldsymbol{\omega}_{0} \cdot \int_{S_{B}} \hat{\phi}\left(\boldsymbol{n} \times \nabla \tilde{\Phi}_{0}\right) \mathrm{d} S= & -\hat{\boldsymbol{I}} \cdot \frac{\boldsymbol{A}}{\mathscr{V}_{B}} \cdot\left\{\boldsymbol{\omega}_{0} \times(\boldsymbol{A} \cdot \tilde{\boldsymbol{V}})\right\} \\
& -4 \hat{\boldsymbol{j}} \cdot\left\{(\boldsymbol{K} \cdot \boldsymbol{J}) \cdot\left({ }^{\mathrm{T}} \boldsymbol{K} \cdot \boldsymbol{\Omega}\right):\left(\boldsymbol{\epsilon} \cdot \boldsymbol{\omega}_{0}\right)\right\} .
\end{aligned}
$$

Under the same conditions, the tensor $\boldsymbol{K}$ may also be used to re-express the contribution to $(\mathrm{F} 4)$ involving the third-order tensor $\boldsymbol{O}_{R}$ in the form

$$
\hat{\boldsymbol{j}} \cdot\left\{\boldsymbol{O}_{R}:\left(\boldsymbol{\omega}_{0} \boldsymbol{\Omega}\right)\right\}=\hat{\boldsymbol{j}} \cdot\left\{\boldsymbol{J} \cdot\left(\boldsymbol{\omega}_{0} \cdot{ }^{\mathrm{T}} \boldsymbol{K} \cdot \boldsymbol{\Omega}\right)-(\boldsymbol{\Omega} \cdot \boldsymbol{K}) \cdot\left(\boldsymbol{J} \cdot \boldsymbol{\omega}_{0}\right)\right\}
$$

\section{REFERENCES}

Auton, T. R. 1987 The lift force on a spherical body in rotational flow. J. Fluid Mech. 183, $199-218$.

Auton, T. R., Hunt, J. C. R. \& Prud'homme, M. 1988 The force exerted on a body moving in an inviscid unsteady non-uniform rotational flow. J. Fluid Mech. 197, 241-257.

Batchelor, G. K. 1964 Axial flow in trailing line vortices. J. Fluid Mech. 20, 645-658.

Batchelor, G. K. 1967 An Introduction to Fluid Dynamics. Cambridge University Press.

Biesheuvel, A. \& Hagmeijer, R. 2006 On the force on a body moving in a fluid. Fluid Dyn. Res. 38, 716-742.

Brenner, H. 1963 The Stokes resistance of an arbitrary particle. Chem. Engng Sci. 18, 1-25.

Burgers, J. M. 1920 On the resistance of fluids and vortex motion. Proc. K. Akad. Wet. Amsterdam 23, 774-782.

Chan, C.-H. \& LEAL, L. G. 1979 The motion of a deformable drop in a second-order fluid. J. Fluid Mech. 92, 131-170.

Chang, C. C. 1992 Potential flow and forces for incompressible viscous flow. Proc. R. Soc. Lond. A $437,517-525$.

Chang, C. C. \& Chern, R. L. 1991 Vortex shedding from an impulsively started rotating and translating circular cylinder. J. Fluid Mech. 233, 265-298.

Chang, C. C., Yang, S. H. \& ChU, C. C. 2008 A many-body force decomposition with applications to flow about bluff bodies. J. Fluid Mech. 600, 95-104. 
Cox, R. G. \& Brenner, H. 1968 Lateral migration of solid particles in Poiseuille flow. I. Theory. Chem. Engng Sci. 23, 147-173.

DÉRIAT, E. 2002 Inviscid shear flow around a cylinder close to a wall. C. R. Mec. 330, 35-38.

EAMES, I. 2010 Momentum conservation and condensing vapour bubbles. Trans. ASME: J. Heat Transfer 132, 091501.

EAMES, I. \& HUNT, J. C. R. 1997 Inviscid flow around bodies moving in weak density gradients without buoyancy effects. J. Fluid Mech. 353, 331-355.

Galper, A. \& Miloh, T. 1994 Generalized Kirchhoff equations for a deformable body moving in a weakly non-uniform flow field. Proc. R. Soc. Lond. A 446, 169-193.

GAlper, A. \& Miloh, T. 1995 Dynamic equations of motion for a rigid or deformable body in an arbitrary non-uniform potential flow field. J. Fluid Mech. 295, 91-120.

Grotta Ragazzo, C. \& TABAK, E. 2007 On the force and torque on systems of rigid bodies: a remark on an integral formula due to Howe. Phys. Fluids 19, 057108.

Harper, J. F. \& Moore, D. W. 1968 The motion of a spherical liquid drop at high Reynolds number. J. Fluid Mech. 32, 367-391.

Ho, B. P. \& LEAL, L. G. 1974 Inertial migration of rigid spheres in two-dimensional unidirectional flows. J. Fluid Mech. 65, 365-400.

Howe, M. S. 1989 On unsteady surface forces, and sound produced by the normal chopping of a rectilinear vortex. J. Fluid Mech. 206, 131-153.

Howe, M. S. 1991 On the estimation of sound produced by complex fluid-structure interactions, with application to a vortex interacting with a shrouded rotor. Proc. R. Soc. Lond. A 433, 573-598.

Howe, M. S. 1995 On the force and moment on a body in an incompressible fluid, with application to rigid bodies and bubbles at high and low Reynolds numbers. Q. J. Mech. Appl. Maths 48, 401-426.

Howe, M. S. 2007 Hydrodynamics and Sound. Cambridge University Press.

Howe, M. S., LAUchle, G. C. \& WANG, J. 2001 Aerodynamic lift and drag fluctuations of a sphere. J. Fluid Mech. 206, 131-153.

KAMBE, T. 1987 A new expression of force on a body in viscous vortex flow and asymptotic pressure field. Fluid Dyn. Res. 2, 15-23.

KANG, I. S. \& LEAL, L. G. 1988 The drag coefficient for a spherical bubble in a uniform streaming flow. Phys. Fluids 31, 233-237.

Kochin, N. E., Kibel, I. A. \& Roze, N. V. 1964 Theoretical Hydromechanics. Wiley.

KoK, J. B. W. 1992 Dynamics of a pair of gas bubbles moving through liquid. Part I. Theory. Eur. J. Mech. (B/Fluids) 12, 515-540.

KorotKin, A. I. 2009 Added-Masses of Ship Structures. Springer.

LAMB, H. 1945 Hydrodynamics. Cambridge University Press.

Legendre, D. \& MAgnaudet, J. 1998 The lift force on a spherical bubble in a viscous linear shear flow. J. Fluid Mech. 368, 81-126.

Legendre, D., Magnaudet, J. \& Mougin, G. 2003 Hydrodynamic interactions between two spherical bubbles rising side by side in a viscous liquid. J. Fluid Mech. 497, 133-166.

Levich, V. G. 1949 Bubble motion at high Reynolds numbers. Zh. Eksp. Teor. Fiz. 19, 18-24.

LeVICH, V. G. 1962 Physico-Chemical Hydrodynamics. Prentice Hall.

Lighthill, M. J. $1986 a$ An Informal Introduction to Theoretical Fluid Mechanics. Oxford University Press.

Lighthill, M. J. $1986 b$ Fundamentals concerning wave loading on offshore structures. J. Fluid Mech. 173, 667-681.

LORENTZ, H. A. 1907 Ein allgemeiner satz, die bewegung einer reibenden flüssigkeit betreffend, nebst einigen anwendungen desselben (A general theorem concerning the motion of a viscous fluid and a few applications from it). Abh. Theor. Phys. 1, 23-42.

Magnaudet, J. \& EAmes, I. 2000 The motion of high-Reynolds-number bubbles in inhomogeneous flows. Annu. Rev. Fluid Mech. 32, 659-708.

Magnaudet, J. \& Mougin, G. 2007 Wake instability of a fixed spheroidal bubble. J. Fluid Mech. 572, 311-337. 
Magnaudet, J., TAkagi, S. \& Legendre, D. 2003 Drag, deformation and lateral migration of a buoyant drop moving near a vertical wall. J. Fluid. Mech. 476, 115-157.

Milne-Thomson, L. M. 1968 Theoretical Hydrodynamics. Macmillan.

MILOH, T. 2003 The motion of solids in inviscid uniform vortical fields. J. Fluid Mech. 479, $279-305$.

MiLOH, T. 2004 Fluid-body interaction in the presence of uniform vorticity and density gradient. Phys. Fluids 16, 22-28.

Moore, D. W. 1963 The boundary layer on a spherical gas bubble. J. Fluid Mech. 16, 161-176.

MoOre, D. W. 1965 The velocity of rise of distorted gas bubbles in a liquid of small viscosity. J. Fluid Mech. 23, 749-766.

Mougin, G. \& Magnaudet, J. 2002 The generalized Kirchhoff equations and their application to the interaction between a rigid body and an arbitrary time-dependent viscous flow. Intl $J$. Multiphase Flow 28, 1837-1851.

PAlierne, J. F. 1999 On the motion of rigid bodies in incompressible inviscid fluids of inhomogeneous density. J. Fluid Mech. 393, 89-98.

NocA, F., ShiEls, D. \& JEON, D. 1996 Measuring instantaneous fluid dynamic forces on bodies, using only velocity fields and their derivatives. J. Fluids Struct. 11, 345-350.

NocA, F., ShIEls, D. \& JEON, D. 1999 A comparison of methods for evaluating time-dependent fluid dynamic forces on bodies, using only velocity fields and their derivatives. J. Fluids Struct. 13, 551-578.

PAN, L. S. \& CHEW, Y. T. 2002 A general formula for calculating forces on a 2-D arbitrary body in incompressible flow. J. Fluids Struct. 16, 71-82.

POZRIKIDIs, C. 1997 Introduction to Theoretical and Computational Fluid Dynamics. Oxford University Press.

Protas, B., Styczek, A. \& NowAKowski, A. 2000 An effective approach to computation of forces in viscous incompressible flows. J. Comput. Phys. 159, 231-245.

Quartapelle, L. \& NAPOlitano, M. 1983 Force and moment in incompressible flows. AIAA J. 21, 911-913.

Saffman, P. G. 1992 Vortex Dynamics. Cambridge University Press.

SHERwoOd, J. D. 2001 Steady rise of a small spherical gas bubble along the axis of a cylindrical pipe at high Reynolds number. Eur. J. Mech. (B/Fluids) 20, 399-414.

Stone, H. A. \& SAmuel, A. D. T. 1996 Propulsion of microorganisms by surface distortions. Phys. Rev. Lett. 77, 4102-4104.

TAYLOR, G. I. 1928 The forces on a body placed in a curved or converging stream of fluid. Proc. R. Soc. Lond. A 120, 260-283.

TOLLMIEN, W. 1938 Über kräfte und momente in schwach gekrümmten oder konvergenten strömungen. Zng.-Arch. 9, 308-326.

VAsseur, P. \& Cox, R. G. 1976 The lateral migration of a spherical particle in two-dimensional shear flows. J. Fluid Mech. 78, 385-413.

VAN WIJNGAARDEN, L. 2005 Bubble velocities induced by trailing vortices behind neighbours. J. Fluid Mech. 541, 203-229.

WU, J. C. 1981 Theory for aerodynamic force and moment in viscous flows. AIAA J. 19, 432-441. 